\title{
An Interval Constraint System for Lattice Domains
}

\author{
ANTONIO J. FERNÁNDEZ \\ University of Málaga \\ and \\ PATRICIA M. HILL \\ University of Leeds
}

\begin{abstract}
We present a generic framework for defining and solving interval constraints on any set of domains (finite or infinite) that are lattices. The approach is based on the use of a single form of constraint similar to that of an indexical used by CLP for finite domains and on a particular generic definition of an interval domain built from an arbitrary lattice. We provide the theoretical foundations for this framework and a schematic procedure for the operational semantics. Examples are provided that illustrate how new (compound) constraint solvers can be constructed from existing solvers using lattice combinators and how different solvers (possibly on distinct domains) can communicate and hence, cooperate in solving a problem. We describe the language $\mathrm{cl} p(\mathcal{L})$, which is a prototype implementation of this framework and discuss ways in which this implementation may beimproved.

Categories and Subject Descriptors: D.3.2 [Programming Languages]: Language Classification-Constraint and logic languages; D.3.3 [Programming Languages]: Language Constructs and Features-Constraints; F.3.2 [Logics and Meanings of Programs]: Semantics of Programming Languages-Operational semantics
\end{abstract}

General Terms: Algorithms, Languages, Theory

Additional Key Words and Phrases: Constraint, cooperation, indexicals, lattice, propagation

\section{INTRODUCTION}

Constraint Logic Programming (CLP) systems support many different domains such as finiteranges of integers [Carlsson et al. 1997; Codognet and Diaz 1996a], reals [J affar et al. 1992; Refalo and Van Hentenryck 1996; Sidebottom and Havens 1992; Benhamou 1995], finite sets [Walinsky 1989; Gervet 1997], or the Booleans [Codognet and Diaz 1996b; Barth and Bockmayr 1996]. The choice of

This work was supported by EPSRC grants GR/L 19515 and GR/M 05645; the first author was also supported by projects TIC2001-2705-C03-02 and TIC2002-04498-C05-02 funded by the Spanish Ministry of Science and Technology.

Authors' addresses: A. J . Fernández, Departamento de Lenguajes y Ciencias de la Computación, E.T.S.I.I., Universidad de Málaga, 29071 Teatinos, Málaga, Spain; email: afdez@cc.uma.es; P. M. Hill, School of Computing, University of Leeds, Leeds, LS2 9J T, United Kingdom; email: hill@comp.leeds.ac.uk.

Permission to make digital/hard copy of part or all of this work for personal or classroom use is granted without fee provided that the copies are not made or distributed for profit or commercial advantage, the copyright notice, the title of the publication, and its date appear, and notice is given that copying is by permission of ACM, Inc. To copy otherwise, to republish, to post on servers, or to redistribute to lists requires prior specific permision and/or a fee.

(C) 2004 ACM 0164-0925/04/0100-0001 $\$ 5.00$ 
domain determines the nature of the constraints and their solvers; whether or not a domain is discrete or continuous as well as its cardinality influence the constraint solving procedures so that, for example, existing CLP systems have distinct constraint solving methods for the finite and the infinite domains. In practice, constraint problems are often not specific to any particular system domain and thus their formulation has to be artificially adapted to fit a given solver.

Most constraint solvers, called black box solvers, have the control fixed by the system. This black box approach enables very efficient implementations and can provide practical tools for the common constraint applications. However, such black box solvers lack adaptability for use in solving nonstandard problems. To overcome this lack of flexibility, many constraint systems provide glass box constraints [Frühwirth 1998; Codognet and Diaz 1996a]. These allow new constraints to be defined by the user. In this paper, we are particularly interested in glass box systems that do not require the user to have a detailed knowledge of the implementation.

From this perspective, there have been two main separate devel opments for the provision of glass box constraints: the constraint system clp(FD) [Codognet and Diaz 1996a] and the Constraint Handling Rules (CHRs) [Frühwirth 1998]. The first of these, designed for the finite domain (FD) of integers, is based on a single generic constraint that allows the user to define and control the constraint propagation. These constraints, often referred to as indexicals, are very efficient [Fernández and Hill 2000] since the implementation uses a simple interval narrowing technique which can be smoothly integrated into the WAM [Aït-kaci 1999; Diaz and Codognet 1993]. As a result, clp(FD) is now part of mainstream CLP systems such as SICStus Prolog [Carlsson et al. 1997], IF/Prolog [If/Prolog 1994], and GNU Prolog [Diaz and Codognet 2001]. On the other hand, the CHRs (now included as a library in SICStus Prolog [Carlsson et al. 1997]) enable the creation of new user-defined domains and their solvers and allow any interaction between them. Unfortunately the flexibility of these rules has an efficiency cost and the CHR systems have not been able to compete with other systems that employ the more traditional approaches [Fernández and Hill 2000].

It follows from this discussion that what is needed is a glass box system that combines the flexibility of CHRs with the efficiency of $\mathrm{clp}(\mathrm{FD})$. For this reason, wehave adopted the indexical approach of $c l p(F D)$ to constraint solving, generalizing it for any set of domains that are lattices, thereby providing a flexibility closer to that of CHRs. Our framework has many advantages and, we believe, considerable potential as indicated below.

- The only condition we have placed on a domain is that it must be a lattice. Since, as far as we know, all existing domains provided for CLP systems are already lattices or could be easily extended to become lattices, it can support a wide variety of applications. Moreover, lattice combinators such as the direct and lexicographic products can be used to combine existing domains and their constraint operations to form the basis of new (compound) solvers. 
- The framework is defined on a set of domains, allowing information to flow between domains. This provides an appropriate setting for solving (probably new) applications defined in the interval domain and over which different solvers, possibly on distinct domains, can communicateand, hence, cooperate.

- The basic schema for solving constraints is uniform over all domains regardless of whether they are user-defined or system-defined and irrespective of their cardinality. We prove that any constraint solver that is an instance of this schema is correct and show how termination may be ensured.

- Our framework is based on the indexical approach. Thus we anticipate that adaptations of the techniques used for the implementation of $\mathrm{clp}(\mathrm{FD})$ can be used for the implementation of our operational schema, thereby obtaining a reasonable efficiency compared with other CLP systems.

The paper is based on the Ph.D. dissertation of A. J . Fernández [Fernández 2002]; more information about the theory and/or the $\mathrm{cl}(\mathcal{L})$ language/system is availablethere. Furthermore, the constraint framework described in this paper is a simpl ified and improved version of that given in Fernández and Hill [1999b]. In Section 2, we describe the al gebraic concepts used in the paper. In Section 3, we define the computation domain and construct the interval domain used for constraint solving. In Section 4, the interval constraints are presented together with the procedure for constraint propagation and narrowing. In Section 5, we provide a schema for the operational semantics of our constraint solver and show how this can be adapted so as to ensure termination for infinite as well as finite domains. In Section 6, we provide additional nonstandard examples of computation domains and also show how new domains can be constructed using different lattice combinators. We also define high-level constraints and show how these provide support for solver cooperation. An overview of the CLP language $\operatorname{cl}(\mathcal{L})$ that we have implemented to demonstrate the viability of the theoretical framework is described in Section 7. Section 8 deals with several important issues as for example how to solve disjunctive constraints or nonlinear constraints among others. The main part of the paper ends with a discussion about related work and the conclusions. The proofs of all the results stated in the main part of the paper are included in the Appendix.

\section{PRELIMINARIES AND NOTATION}

If $C$ is a set, then \#C denotes its cardinality, $\wp(C)$ its power set, and $\wp_{f}(C)$ the set of all the finite subsets of $C$, that is to say, $\wp_{f}(C)=\{C \in \wp(C) \mid C$ is finite $\}$.

Ordering. Let $\mathrm{C}$ be a set with equality. $\mathrm{A}$ binary relation $\preceq$ on $\mathrm{C}$ is an ordering relation if it is reflexive, antisymmetric, and transitive. Let $C$ be a set with ordering relation $\preceq$ and $c, c^{\prime} \in C$. Then, we write $c \sim c^{\prime}$ if either $c \preceq c^{\prime}$ or $\mathrm{C}^{\prime} \preceq \mathrm{c}$ and $\mathrm{c} \nsucc \mathrm{c}^{\prime}$ otherwise. Also $\mathrm{c} \prec \mathrm{c}^{\prime}$ if $\mathrm{c} \preceq \mathrm{C}^{\prime}$ and $\mathrm{c} \neq \mathrm{C}^{\prime}$. Any set $\mathrm{C}$ for which an ordering relation is defined is said to be ordered. We say $C$ is totally ordered if for any $a, b \in C, a \sim b$.

Bounds. Let $C$ be an ordered set. An element $C$ in $C$ is a lower (upper) bound of a subset $E \subseteq C$ if and only if $\forall x \in E: C \preceq x(x \preceq c)$. If the set of 
lower (upper) bounds of $E$ has a greatest (least) element, then that element is called the greatest lower bound (least upper bound) of $E$ and denoted by $\mathrm{gl} \mathrm{b}_{\mathrm{C}}(\mathrm{E})$ $\left(\operatorname{lub}_{C}(E)\right)$. If $E=\{x, y\}$, wewritegl $b_{C}(x, y)$ to denotegl $b_{C}(\{x, y\})$ and $\operatorname{lub}_{C}(x, y)$ to denote $\operatorname{lub}_{\mathrm{C}}(\{\mathrm{x}, \mathrm{y}\})$.

Predecessor and successor. Let $C$ be an ordered set and let $c, C^{\prime} \in C$. Then $\mathrm{C}=\operatorname{pre}\left(\mathrm{c}^{\prime}\right)$ is an immediate predecessor of $\mathrm{C}^{\prime}$ and $\mathrm{C}^{\prime}=\operatorname{succ}(\mathrm{c})$ an immediate successor of $\mathrm{c}$ if $\mathrm{c} \prec \mathrm{C}^{\prime}$ and for any $\mathrm{C}^{\prime \prime} \in \mathrm{C}$ with $\mathrm{c} \preceq \mathrm{C}^{\prime \prime} \prec \mathrm{C}^{\prime}$ implies $\mathrm{C}=\mathrm{C}^{\prime \prime}$.

Monotonicity. Let $f$ bea n-ary function $f:: C_{1} \times \cdots \times C_{n} \rightarrow C$, where $C$ and all $C_{i}$, for $i \in\{1, \ldots, n\}$, are ordered sets. Then $f$ is monotonic in $C$ if, whenever $t_{i}, t_{i}^{\prime} \in C_{i}$ such that $t_{i} \preceq t_{i}^{\prime}$, for all $i \in\{1, \ldots, n\}$, then

$$
f\left(t_{1}, \ldots, t_{i}, \ldots, t_{n}\right) \preceq f\left(t_{1}^{\prime}, \ldots, t_{i}^{\prime}, \ldots, t_{n}^{\prime}\right) .
$$

A monotonic function $f$ is strict monotonic if, whenever $t_{i}, t_{i}^{\prime} \in C_{i}$ such that $t_{i} \preceq t_{i}^{\prime}$, for all $i \in\{1, \ldots, n\}$ and $t_{j} \prec t_{j}^{\prime}$, for some $j \in\{1, \ldots, n\}$, then

$$
f\left(t_{1}, \ldots, t_{i}, \ldots, t_{n}\right) \prec f\left(t_{1}^{\prime}, \ldots, t_{i}^{\prime}, \ldots, t_{n}^{\prime}\right) .
$$

Lattice Let $L$ be an ordered set. $L$ is a lattice if $\operatorname{lub}_{L}(x, y)$ and $g l b_{L}(x, y)$ exist, for any two elements $x, y \in L$.

Top and bottom elements. Let $L$ be a lattice. If it exists, $g l b_{L}(L)=\perp_{L}$ is the bottom element of $L$. Similarly, if it exists, $l u b_{L}(L)=T_{L}$ is thetop el ement of $L$. The lack of a bottom or top el ement can be remedied by adding a fictitious one. Thus, the lifted lattice of $L$ is $L \cup\left\{\perp_{L}, T_{L}\right\}$ where, if $\mathrm{glb}_{\mathrm{L}}(\mathrm{L})$ does not exist, $\perp_{L}$ is a new element not in $L$ such that $\forall a \in L, \perp_{L} \prec a$ and similarly, if lub $b_{L}(L)$ does not exist, $T_{L}$ is a new element not in $L$ such that $\forall a \in L, a \prec T_{L}$.

Dual. Let $L$ bea lattice. If $a \in L$, then we denoteits dual as â. The dual of $L$, denoted by $\hat{L}$, is the lattice that contains the dual element of any element in $L$, that is to say, $\hat{L}=\{\hat{a} \mid a \in L\}$, and where the ordering is reversed with respect $L$, that is to say, if $a, b \in L$, then $\hat{a} \preceq \hat{b}$ if and only if $b \preceq a$. As consequence, $\hat{L}$ is the lattice that contains exactly the same el ements as $L$ and that is obtained by interchanging $\operatorname{gl}_{\mathrm{L}}(\mathrm{a}, \mathrm{b})$ and $\operatorname{lub}_{\mathrm{L}}(\mathrm{a}, \mathrm{b})$ for any $\mathrm{a}, \mathrm{b} \in \mathrm{L}$. The duality principle for lattices is "the dual of a statement about lattices phrased in terms of glb and lub can be obtained simply by interchanging glb and lub."

Products. Let $L_{1}$ and $L_{2}$ be two (lifted) lattices. Then the direct product $\left\langle L_{1}, L_{2}\right\rangle$ and the lexicographic product $\left(L_{1}, L_{2}\right)$ are lattices where

$$
\begin{aligned}
g l b\left(\left\langle x_{1}, x_{2}\right\rangle,\left\langle y_{1}, y_{2}\right\rangle\right)=\langle & \left.g l b_{L_{1}}\left(x_{1}, y_{1}\right), g l b_{L_{2}}\left(x_{2}, y_{2}\right)\right\rangle ; \\
g l b\left(\left(x_{1}, x_{2}\right),\left(y_{1}, y_{2}\right)\right)= & \text { if } x_{1}=y_{1} \text { then }\left(x_{1}, g l b_{L_{2}}\left(x_{2}, y_{2}\right)\right) \\
& \text { elsif } x_{1} \prec y_{1} \text { then }\left(x_{1}, x_{2}\right) \\
& \text { elsif } x_{1} \succ y_{1} \text { then }\left(y_{1}, y_{2}\right) \\
& \text { else }\left(g l b_{L_{1}}\left(x_{1}, y_{1}\right), T_{L_{2}}\right) ;
\end{aligned}
$$

ACM Transactions on Programming Languages and Systems, Vol. 26, No. 1, J anuary 2004. 
lub is the dual of glb;

$$
\begin{aligned}
& T_{\left\langle L_{1}, L_{2}\right\rangle}=\left\langle T_{L_{1}}, T_{L_{2}}\right\rangle \quad \text { and } \quad \perp_{\left\langle L_{1}, L_{2}\right\rangle}=\left\langle\perp_{L_{1}}, \perp_{L_{2}}\right\rangle ; \\
& T_{\left(L_{1}, L_{2}\right)}=\left(T_{L_{1}}, T_{L_{2}}\right) \text { and } \quad \perp_{\left(L_{1}, L_{2}\right)}=\left(\perp_{L_{1}}, \perp_{L_{2}}\right) .
\end{aligned}
$$

Moreover,

$$
\begin{array}{ll}
\left\langle\mathrm{x}_{1}, \mathrm{y}_{1}\right\rangle \preceq\left\langle\mathrm{y}_{1}, \mathrm{y}_{2}\right\rangle \quad \text { iff } \quad \mathrm{x}_{1} \preceq \mathrm{y}_{1} \text { and } \mathrm{x}_{2} \preceq \mathrm{y}_{2} ; \\
\left(\mathrm{x}_{1}, \mathrm{y}_{1}\right) \preceq\left(\mathrm{x}_{2}, \mathrm{y}_{2}\right) \quad \text { iff } \quad \mathrm{x}_{1} \prec \mathrm{x}_{2} \text { or } \mathrm{x}_{1}=\mathrm{x}_{2} \text { and } \mathrm{y}_{1} \preceq \mathrm{y}_{2} .
\end{array}
$$

For more information about lattices see, for example, Davey and Priestley [1990]. In the rest of the paper, $\left(L, \preceq, g b_{L}, l u b_{L}, \perp_{L}, T_{L}\right)$ denotes a (possible lifted) lattice on $L$ with (possibly fictitious) bounds $\perp_{L}$ and $T_{L}$.

\section{THE COMPUTATION AND INTERVAL DOMAINS}

The domain on which the values are actually computed is called a computation domain. The key aspect of the constraint system described in this paper is that it can be built on any computation domain provided it is a lattice. Throughout the paper, we let $\mathcal{L}$ denote a (possibly infinite) set of computation domains containing at least one element $L$ and let $\hat{\mathcal{L}}=\{\hat{L} \mid L \in \mathcal{L}\}$. With each computation domain $\mathrm{L} \in \mathcal{L}$, we associate a set of variable symbols $V_{L}$ that is disjoint from $V_{L^{\prime}}$ for any $L^{\prime} \in \mathcal{L}$ distinct of $\mathrm{L}$. We define $\mathcal{V}_{\mathcal{L}}=U\left\{V_{\mathrm{L}} \mid \mathrm{L} \in \mathcal{L}\right\}$. It is assumed (without loss of generality) that all $\mathrm{L} \in \mathcal{L}$ are lifted lattices.

Example 1. Most classical constraint domains are lattices. For instance,

$$
\begin{aligned}
& \text { (Integer, } \leq \text {, mini, maxi, } \perp_{\text {Integer }}, T_{\text {Integer }} \text { ), } \\
& \left(\Re, \leq, \text { mini, maxi, } \perp_{\Re}, \top_{\Re}\right. \text { ), } \\
& \text { (Bool, } \Rightarrow, \wedge, \vee, \text { fal se, true), } \\
& \text { (Set } L, \subseteq, \cap, \cup, \emptyset, L \text { ) }
\end{aligned}
$$

are lattices for the integers, reals, Booleans, and sets, respectively, under their usual orders where mini and maxi functions return, respectively, the minimum and maximum element of any two elements in the integers or reals. Note that Integer and $\Re$ are lifted lattices and include the fictitious elements $T_{\text {Integer, }}$, $\perp_{\text {Integer, }} T_{\Re}$, and $\perp_{\Re}$. For the Booleans, it is assumed that Bool $=\{$ false, true $\}$. For the set lattice, we assume that Set $L=\wp(L)$, for each $L \in \mathcal{L}$, where $\mathcal{L}=\{$ Integer, $\Re, B o o l\} \cup\{$ Set $\mathrm{L} \mid \mathrm{L} \in \mathcal{L}\}$. Note that $\mathcal{L}$ is an infinite set of computations domains.

In the rest of the examples in the paper, we will use the computation domains Integer, $\Re$, Bool and Set $L$ for some domain $L \in \mathcal{L}$ without further comment or direct reference to this example.

Although the framework for our constraint system is based on the indexical approach of $\mathrm{dp}(\mathrm{FD})$ to constraint solving which propagates constraints on finite closed intervals, this framework is intended for all lattices including infiniteand continuous ones. For this reason, we need to be able to define the constraints over both open and cl osed intervals. Thus, in therest of this section, wewill show how a computation domain can be combined with a special binary lattice we 
call the bracket domain to form an interval domain suitable for the constraint solving mechanism described in Section 4. We do this in a number of stages. First, in Section 3.1, we define the bracket domain and use this to construct bounded computational domains for the left and right bounds of an interval. In Section 3.2, these are extended to allow for constraint operators. These are further extended in Section 3.3 to include an additional construct called an indexical. In Section 3.4, we use this enhanced bounded domain to construct the interval domain.

\subsection{Bounded Computation Domains}

To define open and closed bounds of the intervals, we first define a domain of brackets.

Definition 1 (Bracket Domain). The bracket domain B is the lattice

$$
\left.\left.\left.\left.\left.\left(\{)^{\prime},{ }^{\prime}\right]\right\}\right\}, \prec, \min _{B}, \max _{B},{ }^{\prime}\right) ', ~ '\right]^{\prime}\right),
$$

where the ordering $\prec$ is defined by ' ' ' $\prec$ ']', and $\min _{B}$ and $\max _{B}$ functions return, respectively, the minimum and maximum of any two elements in $B$.

This domain is combined with any computation domain to form the right and left bounds of an interval.

Definition 2 (SimpleBounded Computation Domain). The simple bounded computation domain for $L$ is the lattice resulting from the lexicographic product $(L, B)$ and denoted by $L^{s}$. An element $(a, b) \in L^{s}$ is denoted by $a_{b}$.

The mirror of $L^{s}$ is the lexicographic product $(\hat{L}, B)$ and is denoted by $\overline{L^{s}}$. The mirror of an element $t=(a, b) \in L^{s}$ is $(\hat{a}, b) \in \overline{L^{s}}$ and denoted by $\bar{t}=\overline{a_{b}}$.

The simple bounded computation domain and its mirror maintain some useful relations that are independent of any specific computation domain. For instance, it follows directly from the definition that $\overline{L^{s}}=\widehat{L^{s}}$. Moreover, if $\mathrm{t}_{1}=\mathrm{ab}_{\mathrm{b}}, \mathrm{t}_{2}=\mathrm{c}_{\mathrm{d}} \in \mathrm{L}^{\mathrm{s}}$, then

$$
\text { if } a \neq c,\left\{\begin{array}{c}
t_{2} \prec t_{1} \Longleftrightarrow t_{1} \prec t_{2} \\
g l b_{L^{s}}\left(t_{1}, t_{2}\right)=\overline{l u b_{L^{s}}\left(t_{1}, t_{2}\right)} \\
l u b_{\overline{L^{s}}}\left(t_{1}, t_{2}\right)=\overline{g l b_{L^{s}}\left(t_{1}, t_{2}\right)}
\end{array}\right\} \text { else }\left\{\begin{array}{l}
t_{1} \preceq t_{2} \Longleftrightarrow t_{1} \preceq t_{2} \\
g l b_{L^{s}}\left(t_{1}, t_{2}\right)=\overline{g l b_{L^{s}}\left(t_{1}, t_{2}\right)} \\
l u b_{L^{s}}\left(t_{1}, t_{2}\right)=\overline{l u b_{L^{s}}\left(t_{1}, t_{2}\right)}
\end{array}\right\} \text {. }
$$

Example 2. When $L=$ Integer, 6 ] denotes $\left.\left(6,{ }^{\prime}\right]^{\prime}\right)$ and $\overline{6}$ ] denotes $\left.\left(\hat{6},{ }^{\prime}\right]^{\prime}\right)$. Also

$$
\begin{aligned}
& 0 \text { ) } \prec 0_{\text {] }} \prec 1_{\text {) }} \prec 1_{\text {] }} \prec \cdots \prec T_{L_{1}} \prec T_{L_{\text {J }}} \text { in Integer }{ }^{\mathrm{S}} \text {, } \\
& \overline{T_{L_{1}}} \prec \overline{T_{L_{l}}} \prec \cdots \prec \overline{1_{1}} \prec \overline{1_{]}} \prec \overline{0_{\text {) }}} \prec \overline{0_{\text {] }}} \text { in } \overline{\text { Integer }^{S}} \text {, } \\
& \left.g l b_{L^{s}}(3,, 5]\right)=\operatorname{lub}_{L^{s}}(3,3)=3 \text {, } \\
& \left.\operatorname{glb}_{\mathrm{L}^{\mathrm{s}}}(\overline{3}, \overline{5]})=\overline{\operatorname{lub}_{L^{s}}(3 \mathrm{3}, 5 \mathrm{j})}=\overline{5]}=\left(\hat{5},{ }^{\prime}\right]^{\prime}\right) \text {, } \\
& \left.\operatorname{lub}_{L^{s}}(\overline{3}, \overline{5]})=\overline{\left.\operatorname{glb}_{L^{s}}\left(3_{]}, 5\right]\right)}=\overline{3_{]}}=\left(\hat{3},{ }^{\prime}\right]^{\prime}\right) \text {. }
\end{aligned}
$$


Moreover, when $L=$ Set Integer, $\{1,3\}$ ) denotes $\left.\left(\{1,3\},{ }^{\prime}\right)^{\prime}\right)$ and $\overline{\{1,3\})}$ denotes $\left.\left(\{1,3\},{ }^{\prime}\right)^{\prime}\right)$. Also

$$
\begin{aligned}
& \{1\}_{]} \prec\{1,3\}_{)} \prec\{1,3\}_{]} \prec\{1,3,5\}_{]} \text {in Set Integer }{ }^{s}, \\
& \overline{\{1,3,5\}_{]}} \prec \overline{\{1,3\})} \prec \overline{\{1,3\}_{]}} \prec \overline{\{1\}_{]}} \text {in Set Integer }{ }^{s}, \\
& \operatorname{glb}_{L^{s}}\left(\{4\}_{]},\{4,6\}_{]}\right)=\{4\}_{]} \text {and } \operatorname{lub}_{L^{s}}\left(\{3\}_{]},\{4,6\}_{]}\right)=\{3,4,6\}_{]}, \\
& \left.\operatorname{lub}_{L^{s}}\left(\overline{\{4\}_{]}}, \overline{\{4,6\})}\right)=\overline{\left.\operatorname{glb}_{L^{s}}\left(\{4\}_{]},\{4,6\}\right]\right)}=\overline{\{4\}_{]}}=\left(\{\hat{4}\},{ }^{\prime}\right]^{\prime}\right) .
\end{aligned}
$$

\subsection{Constraint Operators}

The bounded computation domains are extended to allow for operators.

Definition 3 (Constraint Operators). Suppose $L \in \mathcal{L}$ and $L_{1}, \ldots, L_{n} \in \mathcal{L} \cup \hat{\mathcal{L}}$. Then $\circ$ is called a constraint operator for $L^{s}$ if it is defined as

$$
\begin{gathered}
\circ:: L_{1}^{s} \times \cdots \times L_{n}^{s} \rightarrow L^{s}, \\
\circ\left(a_{1 b_{1}}, \ldots, a_{n} b_{n}\right)=\circ L\left(a_{1}, \ldots, a_{n}\right)_{\circ_{B}\left(b_{1}, \ldots, b_{n}\right),}
\end{gathered}
$$

where $a_{i} \in L_{i}$ and $b_{i} \in B$, for $1 \leq i \leq n$, and $\circ_{L}$ and $\circ_{B}$ are monotonic functions

$$
\begin{gathered}
\mathrm{O}_{\mathrm{L}}:: \mathrm{L}_{1} \times \cdots \times \mathrm{L}_{\mathrm{n}} \rightarrow \mathrm{L}, \\
\mathrm{o}_{\mathrm{B}}: \underbrace{\mathrm{B} \times \cdots \times \mathrm{B}}_{n \text { times }} \rightarrow \mathrm{B},
\end{gathered}
$$

defined in the computation domain $L$ and in the bracket domain, respectively, in such a way that, if ${ }_{\mathrm{L}}$ is not a strict monotonic function then ${ }_{\mathrm{B}}$ is a constant function. The mirror $\bar{o}$ of $\circ$ is then defined as

$$
\begin{gathered}
\bar{o}:: \overline{\mathrm{L}_{1}^{s}} \times \cdots \times \overline{\mathrm{Ls}_{n}^{s}} \rightarrow \overline{\mathrm{Ls}^{\mathrm{s}}}, \\
\bar{o}\left(\bar{t}_{1}, \ldots, \overline{\mathrm{t}}_{\mathrm{i}}, \ldots, \overline{\mathrm{t}_{n}}\right)=\overline{o\left(\mathrm{t}_{1}, \ldots, \mathrm{t}_{\mathrm{i}}, \ldots, \mathrm{t}_{n}\right) .}
\end{gathered}
$$

If $\circ$ is a binary constraint operator, we often use infix notation and write $t_{1} \circ t_{2}$ instead of $\circ\left(t_{1}, t_{2}\right)$.

Example 3. Suppose for any $b_{1}, b_{2} \in B$,

$$
b_{1}+{ }_{B} b_{2}=\min _{B}\left(b_{1}, b_{2}\right), \quad b_{1}{ }_{B} b_{2}=\text { ']' if } b_{1}=b_{2} \text { and ')' otherwise. }
$$

Suppose also that $+\mathrm{L}:: \mathrm{L} \times \mathrm{L} \rightarrow \mathrm{L}$ and $-\mathrm{L}:: \mathrm{L} \times \hat{\mathrm{L}} \rightarrow \mathrm{L}$ are strict monotonic on $\mathrm{L}$. Then the binary constraint operators,+- , and their mirrors are defined as

$$
\begin{aligned}
& +:: L^{s} \times L^{s} \rightarrow L^{s}, \quad-:: L^{s} \times \overline{L^{s}} \rightarrow L^{s}, \\
& a_{b}+c_{d}=(a+L c)_{b+{ }_{B} d}, \quad a_{b}-\bar{c}_{d}=(a-L \hat{c})_{b-{ }_{B} d} \text {, } \\
& \bar{F}:: \overline{\mathrm{L}^{\mathrm{s}}} \times \overline{\mathrm{L}^{\mathrm{s}}} \rightarrow \overline{\mathrm{L}^{\mathrm{s}}}, \quad=:: \overline{\mathrm{L}^{\mathrm{s}}} \times \mathrm{L}^{\mathrm{s}} \rightarrow \overline{\mathrm{L}^{\mathrm{s}}}, \\
& \bar{a}_{b} \mp c_{d}=\overline{(a+L c)_{b+B d}}, \quad \bar{a}_{b}=c_{d}=\overline{(a-L \hat{c})_{b-B d} d} \text {. }
\end{aligned}
$$

ACM Transactions on Programming Languages and Systems, Vol. 26, No. 1, J anuary 2004. 
Consider the case $\mathrm{L} \in\{$ Integer, $\Re\}$; then we define $+\mathrm{L} / 2$ and $-\mathrm{L} / 2$ to return, respectively, the sum and difference of their arguments. For example, when $\mathrm{L}=\Re$, by Equations (1) and (2), we have

$$
\begin{aligned}
3.2)+4.1_{\mathrm{J}}=\left(3.2+_{\Re} 4.1\right)_{)_{\mathrm{B}}\right]}=7.3, & \overline{3.2} \overline{+} \overline{4.1_{]}}=\overline{3.2)+4.1_{]}}=\overline{7.3} . \\
7.3)-\overline{4.1_{]}}=(7.3-\widehat{4.1})_{)_{\mathrm{B}}}=3.2, \quad & \overline{7.3)}=4.1_{]}=\overline{7.3)-\overline{4.1_{]}}}=\overline{3.2} .
\end{aligned}
$$

Observe that we declared $-\mathrm{L}$ with the second argument in the mirror domain of that of the first. This ensures that operators such as - Integer, $-{ }_{\Re}$ are monotonic on both arguments. For instance, if $i \in$ Integer, then, as $\hat{i}$ increases, $10-\hat{i}$ increases but 10 - i decreases. From the operator declarations our framework will ensure that all constraints are propagated monotonically (see Section 4.3).

Suppose that the unary operator trunc is defined as

$$
\begin{aligned}
& \text { trunc }:: \Re^{s} \rightarrow \text { Integer }^{s}, \\
& \text { trunc }\left(a_{b}\right)=\text { trunc }_{\text {Integer }}(a)_{\text {trunc }_{B}(b)},
\end{aligned}
$$

where trunc $c_{\text {Integer }}(a)$ is defined to return the integer part of $a$, for any $a \in \Re$, and $\operatorname{trunc}_{B}(b)=$ ']' for any $b \in B$. Then

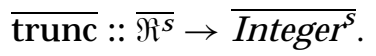

Thus, by Equations (1) and (2),

$$
\begin{aligned}
& \operatorname{trunc}\left(3.1_{)}\right)=\operatorname{trunc}_{\left.\right|_{\text {nteger }}}(3.1)_{\operatorname{trunc}_{B}\left({ }^{\prime}\right)}=3_{\mathrm{J}}, \\
& \overline{\operatorname{trunc}\left(3.1_{)}\right)}=\overline{\operatorname{trunc}\left(3.1_{)}\right)}=\overline{3_{\mathrm{J}}} .
\end{aligned}
$$

Consider now the case $L=$ Set $L^{\prime}$ with $L^{\prime} \in \mathcal{L}$; then we define $+/ 2$ and $-/ 2$ as follows:

$$
\begin{array}{cl}
+:: L^{\mathrm{s}} \times L^{\mathrm{s}} \rightarrow \mathrm{L}^{\mathrm{s}}, & -:: \mathrm{L}^{\mathrm{s}} \times \overline{\mathrm{L}^{\mathrm{s}}} \rightarrow \mathrm{L}^{\mathrm{s}}, \\
\mathrm{a}_{\mathrm{b}}+\mathrm{c}_{d}=(\mathrm{a} \cup \mathrm{c})_{]}, & \mathrm{a}_{\mathrm{b}}-\overline{\mathrm{C}_{d}}=(\mathrm{a} \backslash \hat{\mathrm{c}})_{]} .
\end{array}
$$

Then, for example, for $L^{\prime}=$ Integer,

$$
\begin{aligned}
& \{1,3\}_{]}+\{3,4\}_{)}=(\{1,3\} \cup\{3,4\})_{]}=\{1,3,4\}_{]}, \\
& \overline{\{1,3\}_{]}} \overline{+} \overline{\{3,4\})}=\overline{\{1,3\}]\{3,4\}_{)}}=\overline{\{1,3,4\}_{]},} \\
& \{1,3\}_{]}-\overline{\{3,4\}_{]}}=(\{1,3\} \backslash \overline{\{3,4\}})_{]}=\{1\}_{]}, \\
& \overline{\{1,3\}_{]}}=\{3,4\}_{]}=\overline{\{1,3\}_{]} \backslash \overline{\{3,4\}_{]}}}=\overline{\{1\}]} .
\end{aligned}
$$

In the definition of an operator such as trunc in the previous example, we allowed for its arguments to have a different computation domain from that of theresult. This provides a channel of communication from one solver to another, allowing the information to flow between different domains.

\subsection{Indexicals}

The variables in $V_{L}$ are introduced into the domain $L^{s}$ and its mirror by means of indexicals. Let $\mathrm{O}_{\mathrm{L}}$ be a set of constraint operators defined for $L^{S}$. 
Definition 4 (Bounded Computation Domain). The bounded computation domain (for $\mathrm{L}$ ) $\mathrm{L}^{\mathrm{b}}$ and its mirror $\overline{\mathrm{L}^{\mathrm{b}}}$ are defined as

$$
\begin{aligned}
& \mathrm{L}^{\mathrm{b}}=\mathrm{L}^{\mathrm{s}} \cup\left\{\max (\mathrm{x}) \mid \mathrm{x} \in \mathrm{V}_{\mathrm{L}}\right\} \cup\left\{\operatorname{val}(\mathrm{x}) \mid \mathrm{x} \in \mathrm{V}_{\mathrm{L}}\right\} \\
& \cup\left\{\begin{array}{l|l}
o\left(t_{1}, \ldots, t_{n}\right) & \begin{array}{l}
\circ:: L_{1}^{\prime} \times \cdots \times L_{n}^{\prime} \rightarrow L^{s} \in O_{L}, \\
t_{i} \in\left(L_{i}^{\prime}\right)^{b} \text { for }(1 \leq i \leq n)
\end{array}
\end{array}\right\}, \\
& \overline{\mathrm{L}^{\mathrm{b}}}=\left\{\mathrm{E} \mid \mathrm{t} \in \mathrm{L}^{\mathrm{b}}\right\} \text {, }
\end{aligned}
$$

where

$$
\begin{aligned}
& \text { for } i \in\{1, \ldots, n\},\left(L_{i}^{\prime}\right)^{b}=L_{i}^{b} \quad \text { if } \quad L_{i}^{\prime}=L_{i}^{s} \text { and }\left(L_{i}^{\prime}\right)^{b}=\overline{L_{i}^{b}} \text { if } L_{i}^{\prime}=\overline{L_{i}^{s}} ; \\
& \overline{\max (x)}=\min (x), \overline{\min (x)}=\max (x) \text { and } \overline{\overline{\operatorname{val}(x)}}=\operatorname{val}(x) ; \\
& \text { for each } \circ:: L_{1}^{\prime} \times \cdots \times L_{n}^{\prime} \rightarrow L^{s} \in O_{L}, \\
& \qquad \overline{o\left(t_{1}, \ldots, t_{i}, \ldots, t_{n}\right)}=\bar{o}\left(t_{1}, \ldots, t_{i}, \ldots, t_{n}\right) .
\end{aligned}
$$

The expressions $\max (x), \min (x), \operatorname{val}(x)$, and $\overline{\operatorname{val}(x)}$ are called indexicals.

We define $T_{L^{b}}=T_{L^{s}}$ and $\perp_{L^{b}}=\perp_{L^{s}}$ and the ordering of $L_{b}$ to be inherited from that of $L^{s}$. Thus $L^{b}$ is also a lattice. Note that, if $t \in L^{b}$, then $E \in \overline{L^{b}}$, and, if $\mathrm{t} \in \overline{\mathrm{L}^{\mathrm{b}}}$, then $\mathrm{E} \in \mathrm{L}^{\mathrm{b}}$.

Example 4. Let + (for $L=$ Integer), $-($ for $L=\Re$ ), and trunc be as defined in Example 3. Then + , trunc $\in \mathrm{O}_{\text {Integer }},-\in \mathrm{O}_{\Re}$. Let also $\mathrm{i} \in \mathrm{V}_{\text {Integer }}$ and $\mathrm{r} \in \mathrm{V}_{\Re}$; then

$$
\begin{aligned}
& \text { 3], } \max (i), \quad 3)+\max (i), \quad \operatorname{trunc}(\max (r)) \text { are in Integer }{ }^{b} \text {; } \\
& \text { 20.1), } \max (r), \quad 20.1_{)}-\min (r), \quad \operatorname{val}(r) \quad \text { are in } \Re^{\mathrm{b}} \text {; } \\
& \overline{3}, \quad \min (i), \quad \overline{3}) \mp \min (i), \quad \overline{\operatorname{trunc}}(\min (r)) \quad \text { are in } \overline{\operatorname{lnteger}{ }^{b}} \text {; } \\
& \overline{20.1}, \quad \min (r), \quad \overline{20.1})=\max (r), \quad \overline{\operatorname{val}(r)} \quad \text { are in } \overline{\Re^{b}} \text {; }
\end{aligned}
$$

and

$$
\overline{3)+\max (i)}=\overline{3} \overline{\overline{\max (i)}}=\overline{3} \bar{\gamma} \mp \min (\mathrm{i}) .
$$

\subsection{Interval Domains}

We now define the structure over which the constraints will be solved.

Definition 5 (Interval Domain). The interval domain $R_{L}^{b}$ over the domain $L$ is the direct product $\left\langle\overline{L^{b}}, L^{b}\right\rangle$. The simpleinterval domain $R_{L}^{s}$ over $L$ is the direct product $\left\langle\overline{\mathrm{L}^{\mathrm{s}}}, \mathrm{L}^{\mathrm{s}}\right\rangle$.

Note that $R_{L}^{b}$ and $R_{L}^{s}$ are lattices since they are constructed from the direct product of lattices. It is important to note that the ordering $\preceq$ for $R_{L}^{S}$ simulates the interval inclusion. ${ }^{1}$

${ }^{1}$ For instance, $\left.\langle\overline{3.0}, 4.0\rangle, \preceq\langle\overline{1.8}, 4.5]\right\rangle$ intuitively means that $[3.0,4.0) \subseteq(1.8,4.5]$. 
Definition 6 (Range). An element $r \in R_{L}^{b}$ is called a range If $r \in R_{L}^{s}$, then we say it is simple. A simple range $r=\langle\overline{\mathrm{s}}$, $\mathrm{t}\rangle$ is consistent

(1) if $\mathrm{s} \preceq \mathrm{t}$ and

(2) if $s=a$ ) and, for some $b \in B, t=a_{b}^{\prime}$, then $a \neq a^{\prime}$.

Note that (1) implies that $r$ is inconsistent if $s \chi_{L^{s}} t$.

Example 5. In the domains $\Re$, Integer, and Set Integer, with i $\in V_{\Re}$

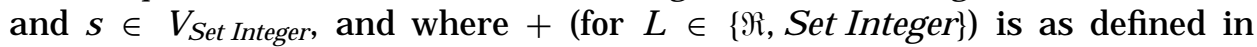
Example 3,

$$
\begin{aligned}
& \langle\overline{2.3}, 8.9)\rangle \in \mathrm{R}_{\Re}^{\mathrm{s}} \text { is consistent; } \\
& \langle\overline{2.3}, 2.2]\rangle \in \mathrm{R}_{\Re}^{\mathrm{s}} \text { is inconsistent; } \\
& \left\langle\overline{1.4}, \max (\mathrm{i})+4.9_{\mathrm{J}}\right\rangle \in \mathrm{R}_{\mathfrak{R}}^{\mathrm{b}} \text {; } \\
& \operatorname{glb}_{R_{\Re}^{b}}(\langle\overline{3.2]}, 6.7 \mathrm{~J}\rangle,\langle\overline{1.8}, 4.5 \mathrm{~J}\rangle)=\left\langle\overline{3.2}, 4.5_{\mathrm{J}}\right\rangle ; \\
& \operatorname{lub}_{\mathrm{R}_{\Re}^{\mathrm{b}}}\left(\left\langle\overline{3.2 \mathrm{]}}, 6.7_{\mathrm{j}}\right\rangle,\left\langle\overline{1.8}, 4.5_{\mathrm{J}}\right\rangle\right)=\langle\overline{1.8}, 6.7 \mathrm{]}\rangle \text {; } \\
& \left\langle\overline{1_{1}}, 10_{]}\right\rangle \in \mathrm{R}_{\text {Integer }}^{\mathrm{S}} \text { is consistent; } \\
& \left\langle\overline{1_{)}}, 1_{]}\right\rangle,\left\langle\overline{5_{]}}, 2_{]}\right\rangle \in R_{\text {lnteger }}^{\mathrm{s}} \text { are inconsistent; } \\
& \langle\overline{\{1\}]},\{1,3\}]\rangle \in R_{\text {Set Integer }}^{S} \text { is consistent; } \\
& \langle\overline{\{1,3\}},\{1\}]\rangle,\langle\overline{\{1,3\}},\{1,4\}]\rangle \in \mathrm{R}_{\text {Set Integer }}^{\text {s }} \text { are inconsistent; } \\
& \left\langle\overline{\{1\}_{]}} \mp \min (\mathrm{s}),\{2,5,7,9\}_{]}\right\rangle \in \mathrm{R}_{\text {Set Integer }}^{\mathrm{b}} \text {. }
\end{aligned}
$$

The next result shows that given an (in)consistent range in the $\mathrm{R}_{\mathrm{L}}^{\mathrm{s}}$ lattice it is possible to identify a part of this lattice where every range is (in)consistent.

Proposition 1. Suppose $r, r^{\prime} \in R_{L}^{S}$, for any $L \in \mathcal{L}$, where $r \preceq r^{\prime}$. If $r^{\prime}$ is inconsistent, then $r$ is al so inconsistent.

Example 6. Suppose $\mathrm{L} \in \mathcal{L}$ and $\mathrm{a}, \mathrm{b}, \mathrm{c} \in \mathrm{L}$ where $\mathrm{a} \prec \mathrm{c} \prec \mathrm{b}$. Figure 1 illustrates the part of lattice $R_{L}^{s}$ constructed from the el ements $a, b$ and $c$. N ote that the nodes within the square are all inconsistent ranges where the rest of nodes are all consistent. The nodes with circles are special cases and are considered in Section 8.1.

\section{THE CONSTRAINT DOMAINS}

Interval constraints, which are the basic elements of the constraint solver, are defined in Section 4.1 by coupling a variable with a range. In Section 4.2, we define constraint stores and show how a solution can be computed using two procedures: constraint stabilization and constraint propagation. Then, in Section 4.3, we explain how our constraint system enforces the monotonic propagation of constraints.

As in previous sections, $L$ denotes any domain in $\mathcal{L}, V_{L}$ the set of variables associated with $\mathrm{L}, \mathcal{V}_{\mathcal{L}}=\cup\left\{\mathrm{V}_{\mathrm{L}} \mid \mathrm{L} \in \mathcal{L}\right\}$, and $\mathrm{R}_{\mathrm{L}}^{\mathrm{b}}$ the interval domain over $\mathrm{L}$. Let $X \in \wp_{f}\left(\mathcal{V}_{\mathcal{L}}\right)$. 


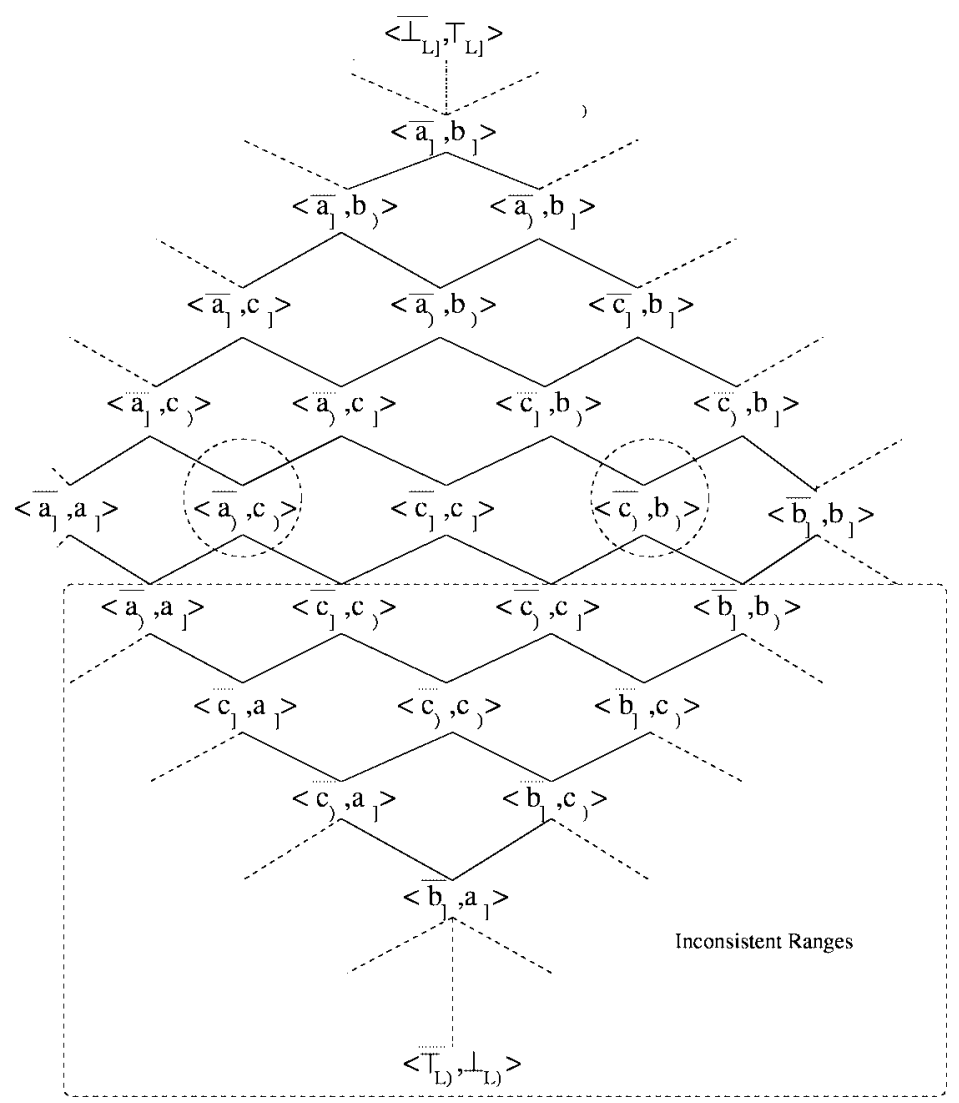

Fig. 1. Structure of the simple interval domain $R_{L}^{S}$ where $a, b, c \in L$ and $a \prec c \prec b$.

\subsection{Interval Constraints}

Definition 7 (Interval Constraint Domain). Suppose $\sqsubseteq:: V_{L} \times R_{L}^{b}$. Then, for all $\mathrm{x} \in \mathrm{V}_{\mathrm{L}}$ and $\mathrm{r} \in \mathrm{R}_{\mathrm{L}}^{\mathrm{b}}$,

$$
\mathrm{c}=\mathrm{x} \sqsubseteq \mathrm{r}
$$

is called an interval constraint for $L$ with constrained variablex. If $r$ is simple (respectively consistent), then $c$ is simple (respectively consistent). If $r=T_{R_{L}^{b}}$, then $c$ is called a type constraint for $x$ and denoted by $x::^{\prime} L$. If $t \in L$, then $x=t$ is a shorthand for $x \sqsubseteq\left\langle\mathrm{t}_{]}, t_{]}\right\rangle$. The interval constraints domain over $X$ for $L$ is the set of all interval constraints for $L$ with constrained variables in $X$ and is denoted by $\mathcal{C}_{\mathrm{L}}^{\mathrm{X}}$. The union

$$
\mathcal{C}^{\mathrm{X}} \stackrel{\text { def }}{=} \bigcup\left\{\mathcal{C}_{\mathrm{L}}^{\mathrm{X}} \mid \mathrm{L} \in \mathcal{L}\right\}
$$

is called the interval constraint domain over $X$ for $\mathcal{L}$.

The ordering for $\mathcal{C}^{\mathrm{X}}$ is inherited from the ordering in $\mathrm{R}_{\mathrm{L}}^{\mathrm{b}}$. We define $\mathrm{C}_{1} \preceq \mathrm{C}_{2}$ if and only if, for some $L \in \mathcal{L}, c_{1}=x \sqsubseteq r_{1}, c_{2}=x \sqsubseteq r_{2} \in \mathcal{C}_{L}^{X}$ and $r_{1} \preceq r_{2}$. 
Definition 8 (Intersection of Simple Interval Constraints). Suppose $x \in X$. The intersection in a domain $\mathrm{L} \in \mathcal{L}$ of two simple constraints $c_{1}, c_{2} \in \mathcal{C}_{L}^{X}$ where $c_{1}=x \sqsubseteq r_{1}, c_{2}=x \sqsubseteq r_{2}$, and $x \in V_{L}$ is defined as follows:

$$
\mathrm{C}_{1} \cap_{\mathrm{L}} \mathrm{c}_{2}=\mathrm{glb}_{\mathcal{C}_{\mathrm{L}}^{\times}}\left(\mathrm{c}_{1}, \mathrm{c}_{2}\right)=\mathrm{x} \sqsubseteq \mathrm{glb}_{\mathrm{R}_{\mathrm{L}}^{\mathrm{s}}}\left(\mathrm{r}_{1}, \mathrm{r}_{2}\right) \text {. }
$$

Suppose $\mathrm{X} \in \mathrm{X}$ and $\mathrm{c}_{1}, \mathrm{c}_{2}, \mathrm{c}_{3} \in \mathcal{C}_{\mathrm{L}}^{\mathrm{X}}$ are simple interval constraints with constrained variable $x$ and $c_{3}=C_{1} \cap_{L} c_{2}$. Then it follows from the definition that $\cap_{\mathrm{L}}$ has the following properties:

Contractance: $c_{3} \preceq c_{1}$ and $c_{3} \preceq c_{2}$;

Correctness: if $\mathrm{c} \preceq \mathrm{c}_{1}$ and $\mathrm{c} \preceq \mathrm{c}_{2}$, then $\mathrm{c} \preceq \mathrm{c}_{3}$;

Commutativity: $\left(\mathrm{C}_{1} \cap_{\mathrm{L}} \mathrm{C}_{2}\right)=\left(\mathrm{C}_{2} \cap_{\mathrm{L}} \mathrm{C}_{1}\right)$;

Idempotence: $\left(c_{1} \cap_{L} c_{3}\right)=c_{3}$ and $\left(c_{3} \cap_{L} c_{2}\right)=c_{3}$.

If $\mathrm{S}_{\mathrm{X}} \subseteq \mathcal{C}_{\mathrm{L}}^{\mathrm{X}}$ is a set of simple constraints with constrained variable $\mathrm{x}$, then we define $\bigcap_{\mathrm{L}} \mathrm{S}_{\mathrm{X}}=\mathrm{glb}_{\mathcal{C}_{\mathrm{L}}}\left(\mathrm{S}_{\mathrm{X}}\right)$. As a result of the contractance property, we have that $\bigcap_{\mathrm{L}} \mathrm{S}_{\mathrm{x}} \preceq \mathrm{C}$, for each $\mathrm{C} \in \mathrm{S}_{\mathrm{x}}$.

Example 7. Examples of theintersection of simple interval constraints are

$$
\begin{aligned}
& \mathrm{i} \sqsubseteq\left\langle\overline{5_{\jmath}}, 24_{\jmath}\right\rangle \cap_{\text {Integer }} \mathrm{i} \sqsubseteq\left\langle\overline{1_{\jmath}}, 14_{\jmath}\right\rangle=\operatorname{glb}_{\mathcal{C}_{\text {Integer }}^{x}}\left(\mathrm{i} \sqsubseteq\left\langle\overline{5_{\jmath}}, 24_{\jmath}\right\rangle, \mathrm{i} \sqsubseteq\left\langle\overline{1_{]}}, 14_{\jmath}\right\rangle\right) \\
& =\mathrm{i} \sqsubseteq \operatorname{glb}_{\mathrm{R}_{\text {Integer }}^{\mathrm{s}}}\left(\left\langle\overline{5_{\mathrm{j}}}, 24_{]}\right\rangle,\left\langle\overline{1_{]}}, 14_{]}\right\rangle\right) \\
& \left.=\mathrm{i} \sqsubseteq\left\langle g l b_{\text {Integer }^{(}}\left(\overline{5_{]}}, \overline{1_{1}}\right), g l b_{\mid \text {nteger }^{s}}\left(24_{]}, 14\right]\right)\right\rangle \\
& =\mathrm{i} \sqsubseteq\langle\overline{5}, 14]\rangle \text {. } \\
& \left.\left.\mathrm{r} \sqsubseteq\langle\overline{1.12]}, 5.67)\rangle \cap_{\Re} \mathrm{r} \sqsubseteq\left\langle\overline{2.34_{]}}, 5.95\right)\right\rangle=\mathrm{r} \sqsubseteq\langle\overline{2.34}, 5.67\rangle\right\rangle ; \\
& \mathrm{b} \sqsubseteq\langle\overline{\text { false }}, \text { true }\rangle \cap_{\text {Bool }} \mathrm{b} \sqsubseteq\langle\overline{\text { false }}, \text { true }\rangle=\mathrm{b} \sqsubseteq\langle\overline{\text { false }}, \text { true }\rangle \text {; } \\
& \left.\left.\mathrm{S} \sqsubseteq\langle\overline{\{1\}},\{1,2,3\}]\rangle \cap_{\text {set Integer }} \mathrm{S} \sqsubseteq\langle\overline{\{2\}},\{1,2,4\}]\right\rangle=\mathrm{S} \sqsubseteq\langle\overline{\{1,2\}},\{1,2\}]\right\rangle \text {. }
\end{aligned}
$$

\subsection{Constraint Stores}

Definition 9 (Constraint Store). If $S \in \wp_{f}\left(\mathcal{C}^{X}\right)$, then $S$ is a constraint store for $X$. If S contains only simple constraints, then it is simple If $S$ is simple, then it is consistent if all its constraints are consistent. The set of all simple constraint stores for $\mathrm{X}$ is denoted by $\mathcal{S}^{\mathrm{X}}$. A constraint store $\mathrm{S}$ is stable if there is exactly one simple constraint for each $\mathrm{x} \in \mathrm{X}$ in $\mathrm{S}$. The set of all simple stable constraint stores for $\mathrm{X}$ is denoted by $\mathcal{S S}^{\mathrm{X}}$.

Let $\mathrm{S}, \mathrm{S}^{\prime} \in \mathcal{S S}^{\mathrm{X}}$ where $\mathrm{C}_{\mathrm{X}}, \mathrm{C}_{\mathrm{x}}^{\prime}$ denote the (simple) constraints for $\mathrm{x} \in \mathrm{X}$ in $\mathrm{S}$ and $S^{\prime}$, respectively. Then $S \preceq S^{\prime}$ if and only if, for each $x \in X, C_{x} \preceq C_{x}^{\prime}$. Let $T_{\mathcal{S S}}{ }^{x}$ be the set of type constraints for $\mathrm{X}$ and $\perp_{\mathcal{S} \mathcal{S}^{\mathrm{X}}}=\left\{\mathrm{X} \sqsubseteq \perp_{\mathrm{R}_{\mathrm{L}}^{\mathrm{s}}} \mid \mathrm{X} \in \mathrm{X} \cap \mathrm{V}_{\mathrm{L}}, \mathrm{L} \in \mathcal{L}\right\}$. Then, with these definitions, $\mathcal{S S}^{\mathrm{X}}$ forms a lattice.

A solution to a constraint store is obtained as a combination of two processes: constraint propagati on and constraint stabilization (i.e., constraint narrowing). The first of this, constraint propagation, is defined by means of an evaluation function. 
Definition 10 (Evaluating Interval Constraints). Let $\mathrm{S} \in \mathcal{S S}^{\mathrm{X}}, \mathrm{x} \in \mathrm{X}$, and let

$$
\begin{aligned}
& \mathcal{L}^{\mathrm{X}}=\cup\left\{\mathrm{L}^{\mathrm{X}} \mid \mathrm{L} \in \mathcal{L}, \mathrm{L}^{\mathrm{X}}=\left\{\mathrm{t} \in \mathrm{L}^{\mathrm{b}} \mid \operatorname{vars}(\mathrm{t}) \subseteq \mathrm{X}\right\}\right\}, \\
& \overline{\mathcal{L}^{\mathrm{X}}}=\cup\left\{\overline{\mathrm{L}^{\mathrm{X}}} \mid \mathrm{L} \in \mathcal{L}, \overline{\mathrm{L}^{\mathrm{X}}}=\left\{\mathrm{t} \in \overline{\mathrm{L}^{\mathrm{b}}} \mid \operatorname{vars}(\mathrm{t}) \subseteq \mathrm{X}\right\}\right\},
\end{aligned}
$$

where vars(t) denotes the set of variables occurring in $t$. Then the (overloaded) evaluation functions are defined:

$$
\begin{aligned}
& \text { eval :: } \mathcal{S S}^{\mathrm{X}} \times \mathcal{L}^{\mathrm{X}} \rightarrow \mathcal{L}^{\mathrm{x}}, \quad \text { eval }:: \mathcal{S S}^{\mathrm{X}} \times \overline{\mathcal{L}^{\mathrm{X}}} \rightarrow \overline{\mathcal{L}^{\mathrm{X}}} \text {, } \\
& \operatorname{eval}(\mathrm{S}, \mathrm{t})=\mathrm{t} \quad \text { if } \mathrm{t} \in \mathrm{L}^{\mathrm{s}} \cup \overline{\mathrm{L}^{\mathrm{s}}}, \mathrm{L} \in \mathcal{L} \text {, } \\
& \operatorname{eval}(S, \max (x))=t \quad \text { where } x \sqsubseteq\langle\bar{S}, t\rangle \in \mathrm{S} \text {, } \\
& \operatorname{eval}(S, \min (x))=s \quad \text { where } x \sqsubseteq\langle s, t\rangle \in S \text {, } \\
& \operatorname{eval}(S, \operatorname{val}(x))=\mathrm{t} \quad \text { if } x \sqsubseteq\langle t, t\rangle \in S \text {, } \\
& \operatorname{eval}(S, \operatorname{val}(x))=\operatorname{val}(x) \quad \text { if } x \sqsubseteq\langle t, t\rangle \notin S \text {, } \\
& \operatorname{eval}(\mathrm{S}, \overline{\operatorname{val}(\mathrm{x})})=\mathrm{E} \quad \text { if } \mathrm{x} \sqsubseteq\langle\mathrm{t}, \mathrm{t}\rangle \in \mathrm{S} \text {, } \\
& \operatorname{eval}(\mathrm{S}, \overline{\operatorname{val}(\mathrm{x})})=\overline{\operatorname{val}(\mathrm{x})} \quad \text { if } \mathrm{x} \sqsubseteq\langle\mathrm{t}, \mathrm{t}\rangle \notin \mathrm{S} \text {, } \\
& \operatorname{eval}\left(S, \circ\left(t_{1}, \ldots, t_{n}\right)\right)=\circ\left(\operatorname{eval}\left(S, t_{1}\right), \ldots, \operatorname{eval}\left(S, t_{n}\right)\right) \text {, } \\
& \operatorname{eval}\left(S, \bar{o}\left(t_{1}, \ldots, t_{n}\right)\right)=\bar{o}\left(\operatorname{eval}\left(S, t_{1}\right), \ldots, \operatorname{eval}\left(S, t_{n}\right)\right) \text {. }
\end{aligned}
$$

Let $s, t \in L^{b}$. Then we further overload eval $/ 2$ and define

$$
\operatorname{eval}(S, x \sqsubseteq\langle s, t\rangle)=x \sqsubseteq\langle\operatorname{eval}(S, s) \text {, eval }(S, t)\rangle .
$$

Definition 11 (Constraint Propagation). Suppose $S \in \mathcal{S S}^{\mathrm{X}}$. If $\mathrm{c}, \mathrm{C}^{\prime} \in \mathcal{C}_{\mathrm{L}}^{\mathrm{X}}$ and eval $(S, C)=C^{\prime}$ is simple, then we say that $c$ is propagated (using $S$ ) to $C^{\prime}$ and write $C \rightsquigarrow^{S} C^{\prime}$. If $C \subseteq \mathcal{C}^{X}$ and $C^{\prime}=\left\{C^{\prime} \mid \exists C \in C . C{ }^{5} C^{\prime}\right\}$, then we say that $C$ is propagated to $C^{\prime}$ (using $S$ ) and write $C{ }^{5} C^{\prime}$.

Note that, if $C$ is a simple constraint store for $X$ and $C \rightsquigarrow{ }^{5} C^{\prime}$, then $C^{\prime}$ is a simple constraint store for $\mathrm{X}^{\prime} \subseteq \mathrm{X}$. Note also that, if $\mathrm{X} \sqsubseteq\langle\overline{\mathrm{S}}, \mathrm{t}\rangle \in \mathrm{S}$ where $\mathrm{s} \neq \mathrm{t}$, then the evaluation function eval applied to val $(x)$ returns val $(x)$ unchanged. Thus the indexical val $(x)$ provides a useful tool for delaying the propagation of constraints.

Constraint stabilization is based on the intersection of simple interval constraints.

Definition 12 (Stabilized Store). Suppose $S \in \mathcal{S}^{\mathrm{x}}, \mathrm{S}^{\prime} \in \mathcal{S S}^{\mathrm{X}}$, and, for each $x \in X, S_{X}=\{c \in S \mid c=x \sqsubseteq r\}$. Then, if $S^{\prime}=\left\{\bigcap_{L} S_{X} \mid L \in \mathcal{L}, x \in X \cap V_{L}\right\}$, we say that $\mathrm{S}^{\prime}$ is the stabilized store of $\mathrm{S}$ and write $\mathrm{S} \mapsto \mathrm{S}^{\prime}$.

Note that, by Definition 7, if $\mathrm{S}_{\mathrm{x}}=\emptyset$, then $\bigcap_{\mathrm{L}} \mathrm{S}_{\mathrm{x}}=\mathrm{x} \sqsubseteq \mathrm{T}_{\mathrm{R}_{\mathrm{L}}^{\mathrm{b}}} \in \mathcal{S S}^{\mathrm{X}}$.

Example 8. Suppose $r, w \in V_{\Re}$ and $i \in V_{\text {Integer }}$. Let also

$$
\begin{aligned}
& \mathrm{S}=\{\mathrm{r} \sqsubseteq\langle\overline{8.3}, 20.4]\rangle, \quad \mathrm{w} \sqsubseteq\langle\overline{1.2}], 10.5)\rangle, \quad \mathrm{i} \sqsubseteq\left\langle\overline{0_{]}}, 10_{\}}\right\rangle, \\
& \mathrm{r} \sqsubseteq\langle\overline{1.0]}, 15.0]\rangle, \quad \mathrm{w} \sqsubseteq\langle\overline{5.6}, 15.3\rangle\rangle, \quad \mathrm{i} \sqsubseteq\langle\overline{2}], 15)\rangle\}, \\
& \left.\left.\mathrm{S}^{\prime}=\left\{\mathrm{r} \sqsubseteq\left\langle\overline{8.3}, 15.0_{]}\right\rangle, \quad \mathrm{w} \sqsubseteq\langle\overline{5.6}, 10.5\rangle\right\rangle, \quad \mathrm{i} \sqsubseteq\langle\overline{2}], 10_{]}\right\rangle\right\} .
\end{aligned}
$$


Then $\mathrm{S} \mapsto \mathrm{S}^{\prime}$. M oreover, consider theoperator trunc/ 1 as defined in Example 3 . Observe that eval $\left(S^{\prime}, \min (w)\right)=\overline{5.6}$, and that real values are propagated to the integer domain via the trunc operator, for example,

$$
\left.\operatorname{eval}\left(S^{\prime}, \operatorname{trunc}(\max (w))\right)=\operatorname{trunc}\left(\operatorname{eval}\left(\mathrm{S}^{\prime}, \max (w)\right)\right)=\operatorname{trunc}(10.5)\right)=10_{\mathrm{J}} \text {. }
$$

Thus

$\{r \sqsubseteq\langle\min (w), 20.4]\rangle, \mathrm{i} \sqsubseteq\langle\overline{\operatorname{trunc}}(\min (w)), \operatorname{trunc}(\max (w))\rangle\} \rightsquigarrow \mathrm{S}^{\prime}$

$$
\{r \sqsubseteq\langle\overline{5.6}, 20.4]\rangle, \mathrm{i} \sqsubseteq\langle\overline{5}, 10 \mathrm{j}\rangle\} \text {. }
$$

A solution is a constraint store that cannot be reduced by means of the propagation or stabilization procedures.

Definition 13 (Solution). Let $C \in \wp\left(\mathcal{C}^{\mathrm{X}}\right)$ be a constraint store for $\mathrm{X}$. A solution for $\mathrm{C}$ is a consistent store $\mathrm{R} \in \mathcal{S S}^{\mathrm{X}}$ where,

$$
\begin{aligned}
C & { }^{R} C^{\prime}, \\
R \cup C^{\prime} & \mapsto R .
\end{aligned}
$$

Sol (C) denotes the set of all solutions for $C$. We say that $G=\operatorname{mgs}(C)$ is a most general solution for $C$ if, for all $R \in \operatorname{Sol}(C), R \preceq G$.

\subsection{Monotonicity of Constraints}

Our approach here has the advantage that it guarantees that the interval constraints are propagated monotonically.

Proposition 2. Let $\mathrm{S}_{1}, \mathrm{~S}_{2} \in \mathcal{S S}^{\mathrm{X}}$ such that $\mathrm{S}_{1}$ and $\mathrm{S}_{2}$ are consistent and $\mathrm{S}_{1} \preceq \mathrm{S}_{2}$ and $\mathrm{C} \in \wp\left(\mathcal{C}^{\mathrm{X}}\right)$ such that

$$
\begin{aligned}
& \mathrm{C} \rightsquigarrow{ }^{\mathrm{S}_{1}} \mathrm{C}_{1} \quad \text { and } \quad \mathrm{S}_{1} \cup \mathrm{C}_{1} \mapsto \mathrm{S}_{1}^{\prime}, \\
& \mathrm{C} \rightsquigarrow{ }^{\mathrm{S}_{2}} \mathrm{C}_{2} \text { and } \mathrm{S}_{2} \cup \mathrm{C}_{2} \mapsto \mathrm{S}_{2}^{\prime} .
\end{aligned}
$$

Then $\mathrm{S}_{1}^{\prime} \preceq \mathrm{S}_{2}^{\prime}$.

Example 9. Consider the definition of the operator - in Example3, when $\mathrm{L}$ is $\Re$ so that $-:: \Re^{\mathrm{S}} \times \overline{\mathfrak{R}^{\mathrm{s}}} \rightarrow \mathfrak{R}^{\mathrm{s}}$. Suppose that $\mathrm{X}=\{\mathrm{X}, \mathrm{y}\} \subseteq \mathrm{V}_{\mathfrak{R}}$ and $\mathrm{S}_{1}, \mathrm{~S}_{2} \in \mathcal{S} \mathcal{S}^{\mathrm{X}}$,

$$
\left.\mathrm{S}_{1}=\left\{\mathrm{y} \sqsubseteq\left\langle\overline{2.0}, 4.0_{]}\right\rangle, \mathrm{x}::^{\prime} \mathfrak{R}\right\} \quad \text { and } \quad \mathrm{S}_{2}=\{\mathrm{y} \sqsubseteq\langle\overline{1.0}, 11.0]\rangle, \mathrm{x}::^{\prime} \mathfrak{R}\right\} \text {. }
$$

Then $\mathrm{S}_{1} \prec \mathrm{S}_{2}$. Let

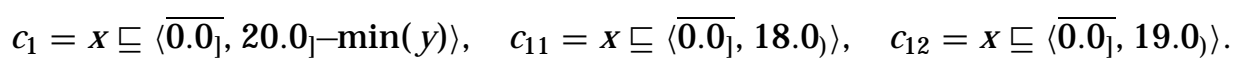

Then $c_{1}$ is an interval constraint for $\Re$ because $\overline{0.0} \in \overline{\Re^{b}}$ and $20.0 \_$min $(y) \in \Re^{b}$ and hence $\left\langle\overline{0.0_{]}}, 20.0_{]}-\min (\mathrm{y})\right\rangle \in \mathrm{R}_{\Re}^{\mathrm{b}}$. Using the constraint propagation procedure for $S_{1}$ and $S_{2}$, we obtain

$$
\mathrm{C}_{1} \rightsquigarrow \mathrm{S} \mathrm{C}_{11}, \quad \mathrm{C}_{1} \rightsquigarrow \mathrm{S}_{2} \mathrm{C}_{12} \text {. }
$$

Note that we have $\mathrm{c}_{11} \prec \mathrm{C}_{12}$.

Second, let

$$
\left.\left.c_{2}=x \sqsubseteq\langle\overline{0.0}, 20.0]-\max (y)\right\rangle, \quad c_{21}=x \sqsubseteq\langle\overline{0.0}, 16.0]\right\rangle, \quad c_{22}=x \sqsubseteq\left\langle\overline{0.0}, 9.0_{]}\right\rangle .
$$

ACM Transactions on Programming Languages and Systems, Vol. 26, No. 1, J anuary 2004. 
procedure solve(C, S)

begin

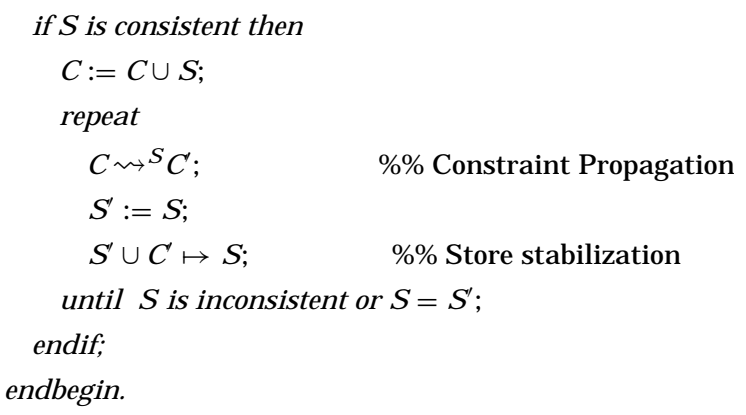

Fig. 2. solve/2: a generic schema for interval constraint propagation.

Then $c_{2}$ is not an interval constraint in our theory. This is because, although $\overline{0.0^{3}} \in \overline{\Re^{\mathrm{b}}}$ and $20.0_{]} \in \mathfrak{R}^{\mathrm{b}}$, we have $\max (\mathrm{y}) \notin \overline{\Re^{\mathrm{b}}}$ with the consequence that, as is defined $-:: \mathfrak{R}^{\mathrm{s}} \times \overline{\mathfrak{R}^{\mathrm{s}}} \rightarrow \mathfrak{R}^{\mathrm{s}}, 20.0_{-}-\max (\mathrm{y}) \notin \mathfrak{R}^{\mathrm{b}}$ and hence $\left\langle\overline{0.0_{]}}, 20.0_{-}-\max (\mathrm{y})\right\rangle \notin$ $\mathrm{R}_{\Re}^{\mathrm{b}}$. Applying the constraint propagation procedure to $c_{2}$ we obtain

$$
\mathrm{C}_{2} \rightsquigarrow \mathrm{S}_{1} \mathrm{C}_{21}, \quad \mathrm{C}_{2} \rightsquigarrow \mathrm{S}_{2} \mathrm{C}_{22} \text {. }
$$

Then we have $c_{22} \prec c_{21}$. Therefore the constraint procedure applied to $c_{2}$ using the smallest constraint store $S_{1}$ derives the largest range for $x$. The problem is caused by the fact that if $S_{2}$ is replaced by a smaller store such as $S_{1}$, $\max (y)$ also decreases in $\Re^{s}$, so that the value of $20.0_{-} \max (y)$ actually increases. Thus, the right bound of the range for $\mathrm{x}$ in $\mathrm{c}_{2}$ also increases so that the upper limit for y can never be reduced.

Note that the acceptability of expressions such as $c_{1}$ and $c_{2}$ as valid constraints can be decided a priori, from the operator declarations, using standard type-checking techniques.

\section{OPERATIONAL SEMANTICS}

In this section, we provide an operational schema for solving the interval constraints and prove both correctness and termination properties. Note that, in this section, the main aim is to provide the basic methodology and we do not discuss possible efficiency improvements.

We continue to use $L$ to denote any domain in $\mathcal{L}, X \in \wp_{f}\left(\mathcal{V}_{\mathcal{L}}\right)$ the set of constrained variables, $\mathcal{C}^{\mathrm{X}}$ the set of all interval constraints for $\mathrm{X}$, and $\mathcal{S S}^{\mathrm{X}}$ the set of all simple stable constraint stores for $X$.

\subsection{Operational Schema}

Let $\mathrm{C} \in \wp_{\mathrm{f}}\left(\mathcal{C}^{\mathrm{X}}\right)$ and $\mathrm{S} \in \mathcal{S S}^{\mathrm{X}}$. We define here solve(C, S), an operational schema for computing a solution (if it exists) for $\mathrm{C} \cup \mathrm{S}$. This schema is shown in Figure 2.

Theorem 1 (Correctness). Let $C \in \wp_{f}\left(\mathcal{C}^{\mathrm{X}}\right)$ and $\mathrm{S} \in \mathcal{S S}^{\mathrm{X}}$. If at least one solution for $\mathrm{C} \cup \mathrm{S}$ exists, then a terminating execution for solve(C, S) returns mgs $(C \cup S)$ in $S$. Otherwise, a terminating execution for solve( $C, S)$ returns in $\mathrm{S}$ an inconsistent store 


\subsection{Termination}

New simple constraints, created by the propagation Step (2) (see Figure 2), are added to the set of constraints before the stabilization Step (4). Thus, with infinite domains, the algorithm may not terminate since the constraints could be contracted indefinitely in the stabilization Step (4).

Example 10. Consider the operator $\operatorname{div} 2:: \mathfrak{R}^{\mathrm{s}} \rightarrow \mathfrak{R}^{\mathrm{s}}$ where $\operatorname{div} Z_{\Re}(\mathrm{a})=\frac{\mathrm{a}}{2.0}$, for any $\mathrm{a} \in \Re$, and $\operatorname{div} 2_{\mathrm{B}}$ is the identity on $\mathrm{B}$. Then let $\mathrm{C}$ be the constraint store

$$
\begin{aligned}
& \{\mathrm{x} \sqsubseteq\langle\overline{0.0}, 10.0 \mathrm{]}\rangle, \mathrm{x} \sqsubseteq\langle\overline{0.0}, \operatorname{div2}(\max (\mathrm{y}))\rangle, \\
& \mathrm{y} \sqsubseteq\langle\overline{0.0}, 10.0]\rangle, \mathrm{y} \sqsubseteq\langle\overline{0.0}, \operatorname{div} 2(\max (\mathrm{x}))\rangle, \\
& \left.\mathrm{Z} \sqsubseteq\left\langle\overline{\perp_{\Re_{i}}}, T_{\Re_{j}}\right\rangle\right\} \text {, }
\end{aligned}
$$

where $\mathrm{x}, \mathrm{y}, \mathrm{z} \in \mathrm{V}_{\Re}$. Let $\mathrm{S}_{0}$ be the top element of the lattice $\mathcal{S} \mathcal{S}^{\{x, y, z\}}$. Let $\mathrm{S}_{i}$ be the value of the store $S$ at the end of the ith iteration for $i \geq 1$ of the operational schema for solve(C, S) with $S_{0}$ the initial value of $S$. Then, in the execution of solve(C, S), S is indefinitely reduced, that is,

$$
\begin{aligned}
& \mathrm{S}_{0}=\left\{\mathrm{X} \sqsubseteq\left\langle\overline{\perp_{\Re_{1}}}, \mathrm{~T}_{\Re_{j}}\right\rangle, \mathrm{y} \sqsubseteq\left\langle\overline{\perp_{\Re_{j}}}, T_{\Re_{1}}\right\rangle, \mathrm{Z} \sqsubseteq\left\langle\overline{\perp_{\Re_{j}}}, T_{\Re_{j}}\right\rangle\right\}, \\
& \mathrm{S}_{1}=\left\{\mathrm{X} \sqsubseteq\left\langle\overline{0.0_{]}}, 10.0_{]}\right\rangle, \mathrm{y} \sqsubseteq\left\langle\overline{0.0_{]}}, 10.0_{\mathrm{j}}\right\rangle, \mathrm{z} \sqsubseteq\left\langle\overline{\perp_{\Re_{1}}}, \mathrm{~T}_{\mathfrak{R}_{\mathrm{j}}}\right\rangle\right\}, \\
& \mathrm{S}_{2}=\left\{\mathrm{X} \sqsubseteq\left\langle\overline{0.0]}, 5.0_{]}\right\rangle, \mathrm{y} \sqsubseteq\left\langle\overline{0.0}, 5.0_{\mathrm{J}}\right\rangle, \mathrm{z} \sqsubseteq\left\langle\overline{\perp_{\Re_{1}}}, \mathrm{~T}_{\Re_{1}}\right\rangle\right\} \text {, } \\
& \mathrm{S}_{3}=\left\{\mathrm{x} \sqsubseteq\left\langle\overline{0.0_{]}}, 2.5_{]}\right\rangle, \mathrm{y} \sqsubseteq\left\langle\overline{0.0_{]}}, 2.5_{]}\right\rangle, \mathrm{z} \sqsubseteq\left\langle\overline{\perp_{\Re_{j}}}, T_{\Re_{j}}\right\rangle\right\},
\end{aligned}
$$

To force termination, we introduce the notion of precision.

Definition 14 (Precision of a Constraint). Let $\mathcal{C C}_{\mathrm{L}}^{\mathrm{X}}$ be the set of all consistent (and thus simple) interval constraints for $L$ with constrained variables in $\mathrm{X}, \mathrm{X} \in \mathrm{X} \cap \mathrm{V}_{\mathrm{L}}$ for any $\mathrm{L} \in \mathcal{L}$, and $\Re \mathcal{I}$ denote the lexicographic product $\left(\Re^{+}\right.$, Integer) where $\Re^{+}$is the (lifted) domain of nonnegative reals. Then we define

$$
\begin{aligned}
& \operatorname{precision}_{\mathrm{L}}:: \mathcal{C C}_{\mathrm{L}}^{\mathrm{X}} \rightarrow \mathfrak{R} \mathcal{I}, \\
& \operatorname{precision}_{\mathrm{L}}\left(\mathrm{x} \sqsubseteq\left\langle\overline{\mathrm{a}_{\mathrm{b}}}, \mathrm{c}_{\mathrm{d}}\right\rangle\right)=\left(\hat{\mathrm{a}} \diamond_{\mathrm{L}} \mathrm{c}, \mathrm{b} \diamond_{B} \mathrm{~d}\right),
\end{aligned}
$$

where $\diamond \mathrm{L}::\{(\hat{a}, \mathrm{c}) \mid \mathrm{a}, \mathrm{C} \in \mathrm{L}, \mathrm{a} \preceq \mathrm{c}\} \rightarrow \mathfrak{R}^{+}$is a (system or user defined) strict monotonic function, and $\diamond_{B}:: B \times B \rightarrow\{0,1,2\}$ is the strict monotonic function

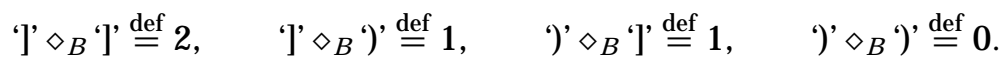

Observe that precision ${ }_{L}$ is defined only on consistent constraints and thus the function $\diamond_{\mathrm{L}}$ only needs to be defined when its first argument is less than or equal to the second. This function must be defined for each computation domain including any fictitious top or bottom elements.

Example 11. Assume that $\Re^{2}=\langle\Re, \Re\rangle$ and suppose that $i_{1}, i_{2} \in$ Integer, $r_{1}, r_{2}, w_{1}, w_{2} \in \Re$, and $s_{1}, s_{2} \in$ Set I nteger where $i_{1} \preceq i_{2}, r_{1} \preceq r_{2}, w_{1} \preceq w_{2}$, and 
$\mathrm{S}_{1} \preceq \mathrm{S}_{2}$. Then

$$
\begin{aligned}
\widehat{i_{1}} \diamond_{\text {Integer }} i_{2} & =i_{2}-i_{1}, \\
\widehat{r_{1}} \diamond_{\Re} r_{2} & =r_{2}-r_{1}, \\
\left.\widehat{r_{1}, w_{1}}\right\rangle \diamond_{\mathfrak{R}^{2}}\left\langle r_{2}, w_{2}\right\rangle & =+\sqrt{\left(r_{2}-r_{1}\right)^{2}+\left(w_{2}-w_{1}\right)^{2}}, \\
\widehat{s_{1}} \diamond \text { set Integer } s_{2} & =\# s_{2}-\# s_{1} .
\end{aligned}
$$

Assume that $\mathrm{i} \in \mathrm{V}_{\text {Integer }}, \mathrm{r} \in \mathrm{V}_{\Re}, \mathrm{y} \in \mathrm{V}_{\Re^{2}}$ and $\mathrm{s} \in \mathrm{V}_{\text {Set Integer }}$. Then

$$
\begin{aligned}
& \text { precision }_{\text {Integer }}\left(i \sqsubseteq\left\langle\overline{1_{]}}, 4_{]}\right\rangle\right)=(3.0,2), \\
& \text { precision } \left._{\Re^{2}}(r \sqsubseteq\langle\overline{3.5}, 5.7)\rangle\right)=(2.2,0), \\
& \text { precision }_{\Re^{2}}\left(y \sqsubseteq\left\langle\overline{(2.0,3.0)_{]}},(3.4,5.6)_{]}\right\rangle\right)=(2.95,2), \\
& \text { precision } \left._{\text {Set Integer }}\left(\mathrm{S} \sqsubseteq\left\langle\overline{\{\}_{]}},\{3,4,5\}\right)\right\rangle\right)=(3.0,1) .
\end{aligned}
$$

Note that the binary operators used in this example, that is, - and + as well as the unary operators \# and "square" need to be defined for both the lifted bounds. The unary operator "square root" must be defined just for the lifted upper bound.

By defining a computable $e^{2}$ bound $\delta \in \Re \mathcal{I}$ (user- or system-defined), we can check if the precision of the simple constraints in a store $\mathrm{S}$ are reduced by a significant amount in thestabilization process (Step 4 in theoperational schema for solve(C, S)). If the change is large enough, then the propagation procedure continues. Otherwise the set of simple constraints in the store $\mathrm{S}$ is considered a "good enough" solution and the procedure terminates. This "solution" is an approximation to the concept of solution shown in Definition 13.

Definition 15. Let $\mathrm{S}, \mathrm{S}^{\prime} \in \mathcal{S S}^{\mathrm{x}}$ be two consistent stores where $c_{\mathrm{x}}, \mathrm{C}_{\mathrm{x}}^{\prime}$ denote the consistent constraints for $\mathrm{x} \in \mathrm{X}$ in $\mathrm{S}$ and $\mathrm{S}^{\prime}$, respectively. Then, we define

no_difference ${ }_{\delta}\left(\mathrm{S}^{\prime}, \mathrm{S}\right) \Longleftrightarrow \forall \mathrm{L} \in \mathcal{L}: \forall \mathrm{X} \in \mathrm{X} \cap \mathrm{V}_{\mathrm{L}}:$

$$
\text { precision }_{\mathrm{L}}\left(\mathrm{c}_{\mathrm{x}}^{\prime}\right)-\text { precision }_{\mathrm{L}}\left(\mathrm{c}_{\mathrm{x}}\right) \preceq \delta \text {, }
$$

where $\left(a_{1}, a_{2}\right)-\left(b_{1}, b_{2}\right)=\left(a_{1}-b_{1}, a_{2}-b_{2}\right)$ and, for $L \in\left\{\right.$ Integer, $\left.\Re^{+}\right\}, x-y$ is defined as usual over any $x, y \in L$ and also $T_{L}-x=T_{L}$ for any $x \in L \cup\left\{\perp_{L}\right\}$ and $T_{L}-T_{L}=0$.

We define a new operational schema for $\operatorname{solve}_{\varepsilon}(C, S)$ which, apart from Step (5) in Figure 2, is the same as the solve(C, S) schema. This step is replaced by:

$\left(5^{\star}\right)$ until $S$ is inconsistent or no_difference ${ }_{\varepsilon, 0)}\left(S^{\prime}, S\right)$ holds.

Theorem 2 (Termination). Let $\mathrm{C} \in \wp_{\mathrm{f}}\left(\mathcal{C}^{\mathrm{X}}\right)$ and $\mathrm{S} \in \mathcal{S S}^{\mathrm{X}}$. If $\varepsilon>0.0$ then the operational schema for $\operatorname{solve}_{\varepsilon}(\mathrm{C}, \mathrm{S})$ terminates.

Definition 16 (ApproximateSolution). Let $\mathrm{C} \in \wp_{\mathrm{f}}\left(\mathcal{C}^{\mathrm{X}}\right)$ and $\mathrm{S} \in \mathcal{S S}^{\mathrm{X}}$. Let also $\mathrm{R}$ be a solution for $\mathrm{C} \cup \mathrm{S}$ and $\delta \in \Re \mathcal{R}$. Then $\mathrm{R}^{\prime}$ is an approximate solution via $\delta$ for $C \cup S$ if $R \preceq R^{\prime}$ and no_difference $\left(R^{\prime}, R\right)$ holds.

${ }^{2}$ That is, representable in the machine which is being used-the computation machine. 
The number of iterations of the operational schema depends, for infinite domains, on the value of $\varepsilon$. In these cases, the final solution for solve $e_{\varepsilon}(C, S)$ is an approximate solution for $\mathrm{C} \cup \mathrm{S}$.

Theorem 3 (Extended Correctness). Let $C \in \wp_{\mathrm{f}}\left(\mathcal{C}^{\mathrm{X}}\right)$ and $\mathrm{S} \in \mathcal{S S}^{\mathrm{X}}$. If at least one solution $\mathrm{R}$ for $\mathrm{C} \cup \mathrm{S}$ exists, a terminating execution of the operational schema for solve $e_{\varepsilon}(\mathrm{C}, \mathrm{S})$ computes in $\mathrm{S}$ an approximate solution for $\mathrm{C} \cup \mathrm{S}$.

The next result shows that the approximate solution is dependent on the value of $\varepsilon$ in the sense that lower $\varepsilon$ is, closer the approximate solution to the solution is.

Theorem 4. Let $\mathrm{R}$ be a solution for $\mathrm{C} \cup \mathrm{S}$ where $\mathrm{C} \in \wp_{\mathrm{f}}\left(\mathcal{C}^{\mathrm{X}}\right)$ and $\mathrm{S} \in \mathcal{S S}^{\mathrm{X}}$. Suppose that $\mathrm{S}_{\varepsilon_{1}}$ and $\mathrm{S}_{\varepsilon_{2}}$ are the approxi mate sol uti ons computed by the operational schema for solve $\varepsilon_{\varepsilon_{1}}(\mathrm{C}, \mathrm{S})$ and $\operatorname{solve}_{\varepsilon_{2}}(\mathrm{C}, \mathrm{S})$, respectively. Then, if $0.0 \leq$ $\varepsilon_{1} \leq \varepsilon_{2}$,

$$
\mathrm{R} \preceq \mathrm{S}_{\varepsilon_{1}} \preceq \mathrm{S}_{\varepsilon_{2}} .
$$

The precision map and the bound $\varepsilon$ allow direct and transparent control over the accuracy of the results. For example, we could define $\varepsilon=10^{-8}$ for reals. Together, the precisi on map and the bound $\varepsilon$ provide a concept of graded solutions to a constraint problem as well as a concept of distance to the correct solution: the higher the bound $\varepsilon$, then the further away (from the correct solution) is the approximate solution. The technique for ensuring termination described here is the first (to our knowledge) fully generic proposal. Note that Sidebottom and Havens [1992] described a similar approach for the real numbers which associates a precision parameter with the real domain and limits the number of times that the domain of a variable may be refined.

\section{APPLICATIONS OF OUR FRAMEWORK}

The I nteger, $\Re$, Bool, and Set $L$ (for any domain $L \in \mathcal{L}$ ) domains have been used throughout the paper to illustrate the concepts as they were defined. In this section, we provide further examples of computation domains, various combinations of the domains using lattice combinators, and a simple extension of the framework to allow for high level constraints.

\subsection{Computation Domains: More Examples}

Strings. Walinsky [1989] described CLP $\left(\Sigma^{*}\right)$, a CLP language that incorporates strings in logic programming by means of membership constraints on finite strings of characters, and indicated the usefulness of this language in a number of diverseareas such as text processing applications or security maintenance in information systems. Our framework can be used to emulate $\operatorname{CLP}\left(\Sigma^{*}\right)$ as follows. Consider the domain String, the set of all sequences (possibly infinite) of characters together with $T_{\text {string. }}$. Assume also the ordering for String is defined such that for $a_{1}, a_{2} \in$ String, $a_{1} \preceq a_{2}$ if and only if $a_{1}$ is a prefix (finite initial substring) of $a_{2}$. Then, letting $\perp_{\text {string }}$ be the empty string, we 
have the lattice

$$
\text { (String, } \preceq, g l b_{\text {String }} \text {, lub } b_{\text {string }}, \perp_{\text {string }}, T_{\text {string}} \text { ). }
$$

Note that $g b_{\text {string }}\left(a_{1}, a_{2}\right)$ is the largest common prefix of $a_{1}, a_{2}$ and lub $b_{\text {string }}\left(a_{1}, a_{2}\right)$ is TString if $a_{1} \nsucc a_{2}, a_{2}$ if $a_{1} \preceq a_{2}$, and $a_{1}$ if $a_{2} \preceq a_{1}$.

Suppose that $++/ 2$ is a constraint operator that concatenates two strings. Let $a, a^{\prime}, a^{\prime \prime} \in V_{\text {String }}$ and

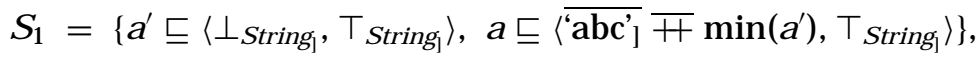

$$
\begin{aligned}
& \mathrm{S}_{2}=\left\{\mathrm{a}^{\prime} \sqsubseteq\left\langle\perp_{\text {string }_{\mathrm{j}}}, \top_{\text {string }}\right\rangle, \mathrm{a}^{\prime \prime} \sqsubseteq\left\langle\perp_{\text {string }_{\mathrm{j}}}, \top_{\text {string }}\right\rangle,\right. \\
& \left.\left.\mathrm{a} \sqsubseteq\left\langle\overline{\operatorname{val}\left(\mathrm{a}^{\prime}\right)} \overline{+} \overline{\left.\mathrm{abc}^{\prime}\right]} \overline{+} \overline{\operatorname{val}\left(\mathrm{a}^{\prime \prime}\right)}, \operatorname{val}\left(\mathrm{a}^{\prime}\right)+{ }^{\prime} \mathrm{abc}^{\prime}\right]+\operatorname{val}\left(\mathrm{a}^{\prime \prime}\right)\right\rangle\right\} \text {. }
\end{aligned}
$$

Then, $S_{1}$ and $S_{2}$ constrains, respectively, $a \in V_{\text {String }}$ to be any string with prefix ' $a b c$ ' and any string containing the string 'abc'.

Nonnegativeintegers ordered by division. Let $\mathcal{N}_{d}$ denote the set of nonnegative integers partially ordered by division: for all $n, m \in \mathcal{N}_{d}, m \preceq n$ if and only if $\exists \mathrm{k} \in \mathcal{N}_{\mathrm{d}}$ such that $\mathrm{km}=\mathrm{n}$ (that is, $\mathrm{m}$ divides $\mathrm{n}$ ). Then

$$
\left(\mathcal{N}_{\mathrm{d}}, \preceq, \mathrm{gcd}, \mathrm{Icm}, 1,0\right)
$$

is a lattice where gcd denotes the greatest common divisor and Icm the least common multiple. Thus with $\mathcal{N}_{d}$ as the computation domain, we have

$$
\mathrm{x} \sqsubseteq\left\langle\overline{2_{\mathrm{J}}}, 24_{\mathrm{J}}\right\rangle \cap_{\mathrm{L}} \mathrm{x} \sqsubseteq\left\langle\overline{3_{\mathrm{J}}}, 36_{\mathrm{J}}\right\rangle=\mathrm{x} \sqsubseteq\left\langle\overline{6_{\mathrm{J}}}, 12_{\mathrm{J}}\right\rangle .
$$

Numeric intervals. We consider Interv as the domain of the numeric intervals. We define $\mathrm{it}_{1} \preceq \mathrm{it} \mathrm{t}_{2}$ if and only if $\mathrm{it}_{1} \subseteq \mathrm{it}_{2}$ (i.e., it ${ }_{1}$ is a subinterval of $\mathrm{it}_{2}$ ). Thus glb $b_{\text {Interv }}$ and lub $b_{\text {Interv }}$ are the intersection and union of intervals, respectively. Our framework solves constraints for the Interv computation domain as follows:

$$
\begin{aligned}
& \left.\mathrm{i} \sqsubseteq\left\langle\overline{[5,6]_{]}},[2,10)_{]}\right\rangle \cap_{\mathrm{L}} \mathrm{i} \sqsubseteq\left\langle\overline{(7,9]_{]}},[4,15]\right]\right\rangle=\mathrm{i} \sqsubseteq\left\langle\overline{[5,6] \cup(7,9]]},[4,10)_{]}\right\rangle . \\
& \text {Note that } \overline{[5,6] \cup(7,9]_{]}} \in \overline{\text { Interv }^{\mathrm{s}}} \text { whereas }[4,10)_{]} \in \text { Interv }^{\mathrm{s}} .
\end{aligned}
$$

\subsection{Combinations of Domains}

Our lattice-based framework allows for new computation domains to be constructed from previously defined domains. Here we give examples which use well-known lattices combinators.

Product of domains. As al ready observed, the direct and lexicographic products of lattices are lattices. For example, consider the lattice Integer.

(1) A point in a plane may be defined by its Cartesian coordinates using the direct product Point $=\langle$ Integer, I nteger $\rangle$.

(2) A rectangle can be defined by two points in a plane: its lower left corner and its upper right corner. Let Rect be the direct product 〈Point, Point〉. 
Interval constraints can be declared directly on these domains. For example, consider $r e \in \mathrm{V}_{\text {Rect; }}$ then

$$
\text { re } \sqsubseteq\left\langle\overline{\langle\langle 2,2\rangle,\langle 5,5\rangle\rangle]},\langle\langle 4,4\rangle,\langle 7,7\rangle\rangle_{]}\right\rangle
$$

constrains the rectangle re to have its lower left corner in the plane $\langle 2,2\rangle \times$ $\langle 4,4\rangle$ and its upper right corner in the plane $\langle 5,5\rangle \times\langle 7,7\rangle$. Thus the rectangle $\langle\langle 3,3\rangle,\langle 6,6\rangle\rangle$ satisfies this constraint.

Sum of domains. The linear sum of $n>1$ lattices is also a lattice.

Definition 17 (Linear Sum). Suppose that $L_{1}, \ldots, L_{n}$ are lattices. Then their linear sum $L_{1} \uplus \cdots \uplus L_{n}$ is the lattice $L_{s}$ where

(1) $\mathrm{L}_{\mathrm{s}}=\mathrm{L}_{1} \cup \cdots \cup \mathrm{L}_{\mathrm{n}}$;

(2) the ordering relation $\preceq$ is defined:

$$
\mathrm{x} \preceq \mathrm{y} \Longleftrightarrow\left\{\begin{array}{l}
\mathrm{x}, \mathrm{y} \in \mathrm{L}_{\mathrm{i}} \text { and } \mathrm{x} \preceq \mathrm{y} \text { or } \\
\mathrm{x} \in \mathrm{L}_{\mathrm{i}}, \mathrm{y} \in \mathrm{L}_{\mathrm{j}} \text { and } \mathrm{i} \prec \mathrm{j} ;
\end{array}\right.
$$

(3) $\mathrm{glb}_{\mathrm{Ls}_{\mathrm{s}}}$ and $l \mathrm{lub}_{\mathrm{L}_{\mathrm{s}}}$ are defined:

$$
\begin{aligned}
& \operatorname{glb}_{L_{s}}(x, y)=g l b_{L_{i}}(x, y) \text { and } \operatorname{lub}_{L_{s}}(x, y)=\operatorname{lub}_{L_{i}}(x, y) \quad \text { if } x, y \in L_{i}, \\
& g^{g} b_{L_{s}}(x, y)=x \text { and } \operatorname{lub}_{L_{s}}(x, y)=y \quad \text { if } x \in L_{i}, y \in L_{j} \text { and } i \prec j, \\
& g^{g l b_{L_{s}}}(x, y)=y \text { and } \operatorname{lub}_{L_{s}}(x, y)=x \quad \text { if } x \in L_{i}, y \in L_{j} \text { and } j \prec i ;
\end{aligned}
$$

(4) $\perp_{L_{s}}=\perp_{L_{1}}$ and $T_{L_{s}}=T_{L_{n}}$.

As an example, consider the lattice AtoF containing all the (uppercase) alphabetic characters between ' $A$ ' and ' $F$ ' with the usual alphabetical ordering and the lattice 0 to9 containing the numeric characters from ' 0 ' to ' 9 ' with the ordering ' 0 ' $<$ ' 1 ' $<\cdots<$ ' 8 ' $<$ ' 9 '. Then the lattice of hexadecimal digits can be defined as the lattice 0 to $9 \uplus \mathrm{AtoF}$. Now, it is possible to constrain variables to have values in such a domain. For example a code of four hexadecimal digits can be initially represented by four variables $h_{1} h_{2} h_{3} h_{4}$ that are constrained by a type constraint as $h_{1}, h_{2}, h_{3}, h_{4}::^{\prime}$ Oto $\uplus \uplus$ AtoF (note that this is equivalent to the constraints $\left.h_{1} \sqsubseteq\left\langle\overline{\left.{ }^{\prime} O^{\prime}\right]},{ }^{\prime} F{ }^{\prime}\right]\right\rangle, h_{2} \sqsubseteq\left\langle\overline{\left.{ }^{\prime} O^{\prime}\right]},{ }^{\prime} F^{\prime}{ }_{j}\right\rangle, h_{3} \sqsubseteq\left\langle\overline{{ }^{\circ} O^{\prime}}{ }^{\prime},{ }^{\prime} F^{\prime}{ }^{\prime}\right\rangle$, and $\left.h_{4} \sqsubseteq\left\langle\overline{{ }^{\prime} O^{\prime}},{ }^{\prime} F^{\prime}{ }_{j}\right\rangle\right)$.

\subsection{High-Level Constraints}

A constraint operator can provide a useful one-way channel of communication by allowing values in the computation domains for its arguments to be propagated to the computation domain in its range. Here we define high-level constraints by means of a generic relation that enables the propagation of information between domains in any direction allowing for full cooperation between the solvers for these domains. So as to distinguish the interval constraints defined and studied in previous sections from the high-level constraints defined here, we call constraints of the form $\mathrm{x} \sqsubseteq \mathrm{r}$ primitive constraints.

Definition 18 (High-Level Constraint). Suppose that $\mathcal{L}^{\prime}=\left\{L_{1}, \ldots, L_{m}\right\} \subseteq$ $\mathcal{L}$. Then $q:: L_{1} \times \cdots \times L_{m}$ is called an $m$-ary constraint relation for $\mathcal{L}^{\prime}$. Suppose $\mathrm{x}_{1} \in \mathrm{V}_{\mathrm{L}_{1}}, \ldots, \mathrm{x}_{\mathrm{m}} \in \mathrm{V}_{\mathrm{L}_{\mathrm{m}}}$, and $\left\{\mathrm{C}_{1}, \ldots, \mathrm{C}_{\mathrm{n}}\right\}$ is a constraint store with constrained 
variables $X \supseteq\left\{x_{1}, \ldots, x_{m}\right\}$. Then

$$
\mathrm{q}\left(\mathrm{x}_{1}, \ldots, \mathrm{x}_{\mathrm{m}}\right) \Leftrightarrow \mathrm{c}_{1}, \ldots, \mathrm{c}_{\mathrm{n}}
$$

is called a high-level constraint over $\mathcal{L}^{\prime}$.

Note that our high-level constraints are similar to dp(FD) [Codognet and Diaz 1996a]. However, unlike clp(FD), our framework also allows for the definition of both generic and overloaded constraints: A high-level constraint is generic for arguments $i_{1}, \ldots, i_{j}\left(1 \leq i_{1}<\cdots<i_{j} \leq m\right)$ if its definition is independent of the choice of domains $L_{i_{1}}, \ldots, L_{i_{j}}$ in $\mathcal{L} ;$; constraint is overloaded for arguments $i_{1}, \ldots, i_{j}$ if it is defined for any $L_{i_{1}}, \ldots, L_{i_{j}}$ in $\mathcal{L}_{1}$ where $\mathcal{L}_{1} \subset \mathcal{L}$ and $\#\left(\mathcal{L}_{1}\right)>1$.

Example 12. Consider the following high-level "less-or-equal" constraint:

$$
\begin{aligned}
x \leq y \Leftrightarrow & x \sqsubseteq\left\langle\overline{\perp_{L_{1}}}, \max (y)\right\rangle, \\
y & \sqsubseteq\left\langle\min (x), T_{L_{3}}\right\rangle .
\end{aligned}
$$

Then this is generic for both arguments of $\leq$ as each $L \in \mathcal{L}$ has (possible lifted) top and bottom elements. Also, consider the definition of the operators + and - shown in Example 3 and the following definition of a plus/3 constraint:

$$
\begin{aligned}
\operatorname{plus}(x, y, z) \Leftrightarrow x & \sqsubseteq\langle\min (z)=\max (y), \max (z)-\min (y)\rangle, \\
y & \sqsubseteq\langle\min (z)=\max (x), \max (z)-\min (x)\rangle, \\
z & \sqsubseteq\langle\min (x) \mp \min (y), \max (x)+\max (y)\rangle .
\end{aligned}
$$

This constraint is overloaded since it is valid for any domain $L$ in which operators $+\mathrm{L}$ and $-\mathrm{L}$ aredefined. For example, a call pl us $(\mathrm{x}, \mathrm{y}, \mathrm{z})$, where $\mathrm{x}, \mathrm{y}, \mathrm{z} \in \mathrm{V}_{\mathrm{L}}$ for $L \in\{$ Integer, $\Re\}$, means $x=y+z$ whereas, for $L=$ Set $L^{\prime}$ and $L^{\prime} \in \mathcal{L}$, it means $(x \cup y=z) \wedge(x \cap y=\emptyset)$.

Note that in an implementation, if $\mathrm{C}$ is a constraint store containing a highlevel constraint $c \Leftrightarrow c_{1}, \ldots c_{n}$, then we replace $c$ (in $C$ ) by $\left\{c_{1}, \ldots, c_{n}\right\}$. This has to be repeated until $C$ contains no high-level constraints. Of course, termination of this is not guaranteed and would depend on the definitions of the high-level constraints. Once $C$ is simplified to a set of primitive constraints, constraint propagation and constraint stabilization can be executed as usual.

\section{A CLP LANGUAGE FOR LATTICES}

In this section, we describe $c \mathrm{p}(\mathcal{L})$ (Constraint Logic Programming on any set $\mathcal{L}$ of lattices), ${ }^{3}$ a language that we have implemented to validate the feasibility of the framework. We also provide a simple scheduling example to illustrate how $\mathrm{cl}(\mathcal{L})$ may be used to solve a constraint problem.

\footnotetext{
${ }^{3}$ Sources, user manual, and a number of $\mathrm{cl} p(\mathcal{L})$ examples areavailablefrom http://www.lcc.uma.es/ afdez/generic. Note that all the examples shown in this paper were tested on this prototype.
} 
7.1 The $c / p(\mathcal{L})$ Language

This language is based on standard Prolog [ISO/I EC 1995], with some extra declarations for specifying both new domains and constraint operators. Thus the set of computation domains $\mathcal{L}$ over which the constraints may be defined is the set of all system and user-declared domains in the program. The advantage of our theoretical approach with respect to other generic approaches (seeSection 9) is that it can be implemented directly from the theory. The interval constraints such as $x \sqsubseteq r$ are expressed in $\operatorname{clp}(\mathcal{L})$ as expressions of the form $X$ isin $R$ and can occur anywhere in the bodies of clauses. A simple range $\left\langle\overline{a_{b}}, c_{d}\right\rangle$ is expressed as an expression (a, bracket(b))..(c, bracket(d)) where bracket(' $\left.]^{\prime}\right)=$ close and bracket $\left.\left({ }^{\prime}\right)^{\prime}\right)=$ open and open and close are reserved words in the system, for example, $\langle\overline{1.2}, 4.5]\rangle$ is denoted as (1.2, open)..(4.5, close).

New domains can be defined by the user with a lattice declaration: the predicate lattice/2 identifies the elements belonging to the new lattice; and the predicates $1 \mathrm{t} / 3, \mathrm{glb} / 4$, and lub/4 define, respectively, the ordering relation, $\mathrm{glb}$, and lub for the domain. That is to say,

- lattice(D, E) is true if $E \in D$;

$-\operatorname{lt}(D, X, Y)$ is true if $X<Y$ in $D$;

$-g \operatorname{lb}(D, X, Y, Z)$ is true if $Z=g b_{D}(X, Y)$;

$-\operatorname{lub}(D, X, Y, Z)$ is true if $Z=\operatorname{lub}_{D}(X, Y)$.

The current prototype implementation of $\operatorname{clp}(\mathcal{L})$ provides some predefined predicates for the combination of domains (e.g., product_Direct/3, linear_sum/3, and product_Lexicographic/3). However, new lattice combinators can be easily implemented in a declarative way via domain declarations. As examples, Figure 3 shows how to declare the set domain (defined as a list with the inclusion as ordering) and the direct product domain reint_point $=\langle$ real, integer $\rangle$ (assuming integer and real are system-defined domains I nteger and $\Re$, respectively).

The language allows the declaration of both unary and binary operators by means of the predicates declara/3 or declara/4. Let $L, L_{1}, L_{2}$ be (user or system) (not necessarily distinct) computation domains. Then

- declara(Op, $\left.L_{1}, L\right)$ specifies the unary operator $O p:: L_{1}^{s} \rightarrow L^{s}$, - declara $\left(O p, L_{1}, L_{2}, L\right)$ specifies the binary operator Op :: $L_{1}^{s} \times L_{2}^{s} \rightarrow L^{s}$.

If $L_{1}$ or $L_{2}$ is replaced in the above by mirror $\left(L_{1}\right)$ or mirror $\left(L_{2}\right)$, then the domains $L_{1}^{s}$ or $L_{2}^{s}$ are replaced by the mirrors $\overline{L_{1}^{S}}$ or $\overline{L_{2}^{s}}$. Figure 4 shows the definition of the operators ${ }^{4}+$ and - of Example 3 over the domains of integers, reals, sets, and reint_point. Observe that these operators are defined on the bracket domain and each of the computation domains (see Definition 3). Particularly, for the set domain, :+: and :-: are defined to be the usual union and difference of sets, respectively.

Theimplementation manages a single constraint store which contains all the (user or system) defined (primitive) interval constraints for domains in $\mathcal{L}$. As in

${ }^{4} \mathrm{By}$ syntax conventions, $\mathrm{Cl}(\mathcal{L})$ operators begin and end with colons.

ACM Transactions on Programming Languages and Systems, Vol. 26, No. 1, J anuary 2004. 


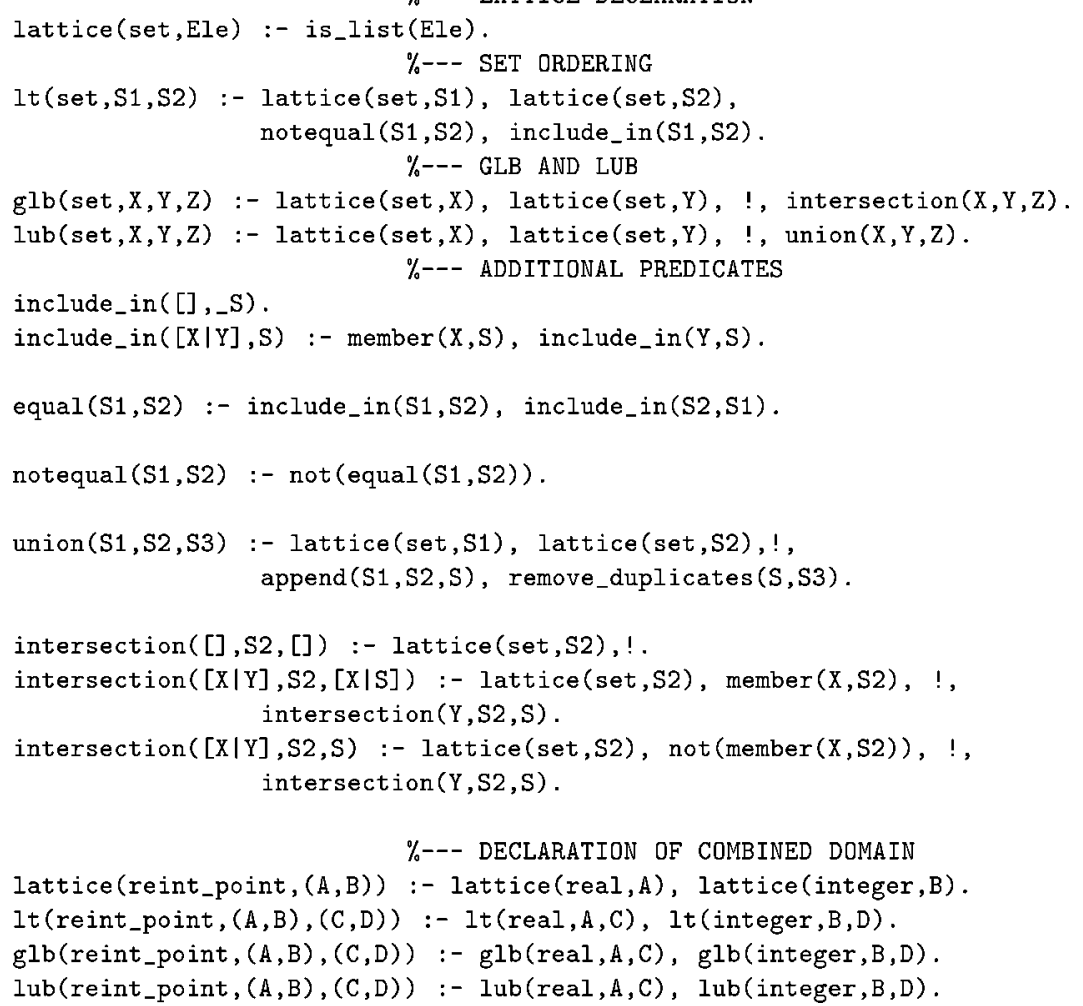

Fig. 3. Lattice declarations: Set $L$ and reint_point.

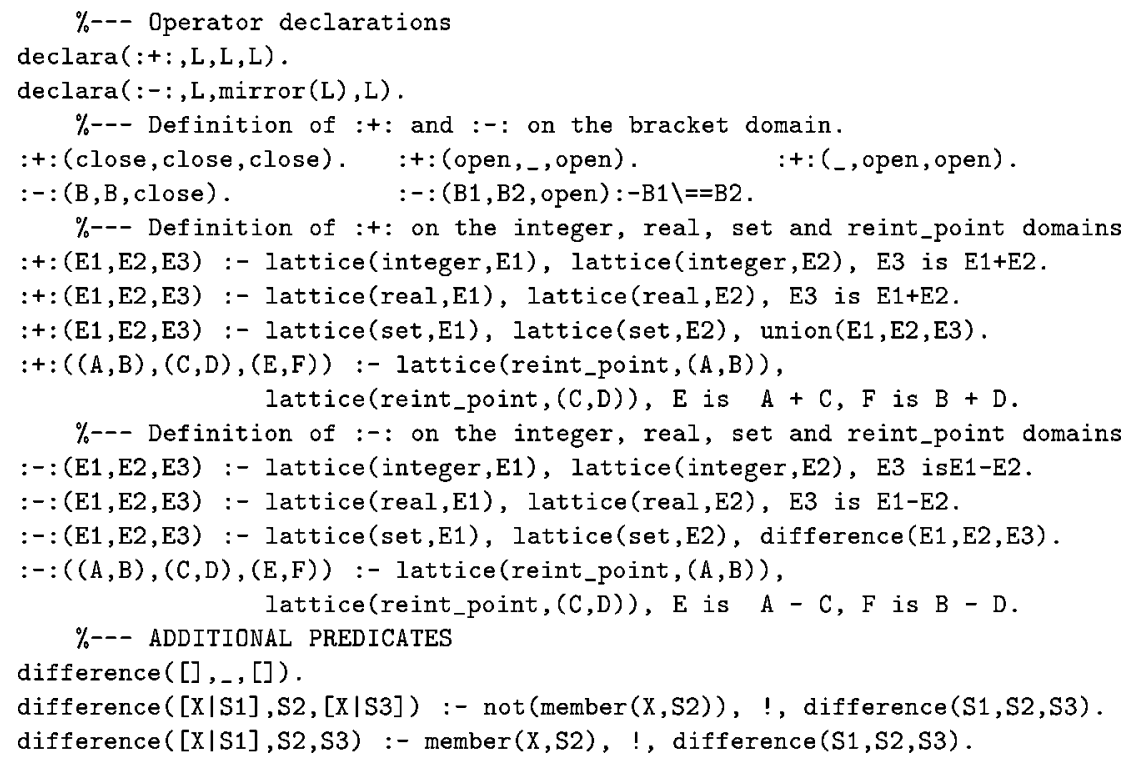

Fig. 4. Operator declarations in $\operatorname{clp}(\mathcal{L})$. 
Prolog, the resolution mechanism of the $\mathrm{d} p(\mathcal{L})$ language is LD-resolution with a special procedure for binding constrained variables. That is, when binding a variable $X$ in a domain $L$ to any term $t$ distinct from $X$, the unification step is

- if $t$ is an unbound variable $Y$, then $Y$ is bound to $X$;

- if $t$ is a term $I \in L$, then the constraint " $X$ isin (, close)..( $($, close)" is added to the store; or

- if t is a constrained variable $Y \in V_{L}$, theconstraints " $X$ isin $\min (Y)$.. $\max (Y)$ " and " $Y$ isin $\min (X) . . \max (X)$ " are added to the store.

Thestep returns a fail, called domain fail, if $t$ is either a constrained variable in $\mathrm{V}_{\mathrm{L}^{\prime}}$ or term in $\mathrm{L}^{\prime}$ and $\mathrm{L}^{\prime} \neq \mathrm{L}$. Also, using the operator declarations, the prototype identifies the nonmonotonic constraints (i.e., constraints not contributing to the solution-see Section 4.3).

Our prototype implementation of $\mathrm{cl} p(\mathcal{L})$ [Fernández 2000] is built on the SICStus 3\#7 Prolog platform [Carlsson et al. 1997]. Constraint consistency, store stabilization, and constraint propagation are implemented using the constraint handling rules (CHRs) [F rühwirth 1998] that are part of a SI CStus library. The CHRs are very appropriate since they are solved prior to the resolution step of the standard logical engine. The current $\mathrm{dlp}(\mathcal{L})$ implementation provides predefined Boolean constraints such as and $/ 3$, or $/ 3$, xor $/ 3$, equiv $/ 3$, and not $/ 2$ among others; symbolic constraints such as at_least_one/1, at_most_one/1, and only_one/1; arithmetic constraints such as plus/3, diff $/ 3$, divide/3, and times $/ 3$ as well as generic arithmetic constraints such as $=/ 2, \neq / 2,>/ 2, \geq / 2,<$ $/ 2$, and $\leq / 2$ defined on usual numerical domains and on combined domains. As $\mathrm{Clp}(\mathcal{L})$ is implemented in SICStus, the prototype supports many system predicates provided by SICStus.

Example 13. Figure 5 shows how to code the high-level constraints in Example 12 as well as how the overloaded plus constraint is used. ${ }^{5}$

\subsection{An Overloaded Generic Scheduling Problem}

This example scheduling problem illustrates the generic power of our solver ${ }^{6}$ where the tasks are represented by terms of the form Task(S, D); $S$ is the start time and $D$ is the duration. The high-level constraint into(Task, SuperTask) is true if the interval for SuperTask contains the interval for Task; noOverlap(Task, Tasks) is true if Task overlaps with no elements in

\footnotetext{
${ }^{5} \operatorname{In} \mathrm{clp}(\mathcal{L}), x::$ ' $L$ denotes a type constraint for $x$ in $L$ (see Definition 7 ) and $\left[x_{1}, \ldots, x_{n}\right]::$ ' $L$ is equivalent to $x_{1}:: ' L, \ldots, x_{n}:: ' L$. bottom and top are reserved words denoting fictitious bottom and top elements for any lattice. Observe also that the constraint $\mathrm{T}$ is in $(3, \mathrm{close}) . .(11$, open) was reduced to the constraint $\mathrm{T}$ is in $(3, \mathrm{close}) . .(10, \mathrm{close})$ by applying the equivalence rules for discrete domains we will describe in Section 8. Note that the prompt is clp (L) >.

${ }^{6}$ This example is a generalization of a program proposed in Sidebottom and Havens [1992] formulated for the real domain and using some of the relations on temporal intervals described in Allen [1983].
}

ACM Transactions on Programming Languages and Systems, Vol. 26, No. 1, J anuary 2004. 


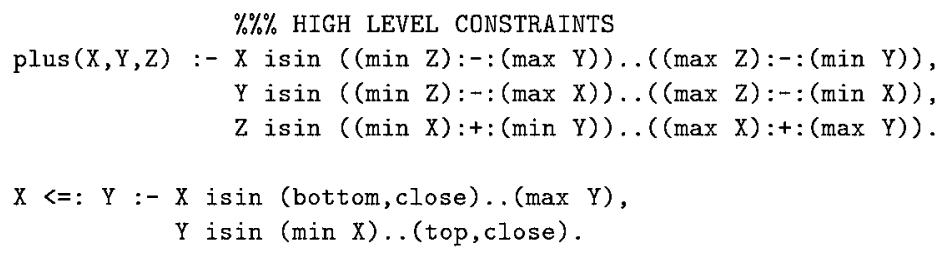

Fig. 5. Using the overloaded constraint plus/3.

Tasks; schedule(Tasks, SuperTask) is true if every task in Tasks is in SuperTask and no pair overlaps.

Figure 6 shows code for solving this problem and provides an application of the generic and overloading capabilities of $\mathrm{clp}(\mathcal{L})$. (Observe that the constraints into/2, noOverlap/2, and schedule/2 are overloaded since they are defined in terms of both the overloaded constraint plus $/ 3$ and the generic constraint $<=: / 2$ that are al ready defined in Figure 5.) Two of the instances use the FD and real domains. The third instance is more interesting and uses the combined domain reint_point as declared in preceding section. Suppose there are two processes $\mathrm{p} 1=(\mathrm{S} 1, \mathrm{D} 1)$ and $\mathrm{p} 2=(\mathrm{S} 2, \mathrm{D} 2)$ where $\mathrm{p} 1$ must be executed on a machine $\mathrm{A}$ in real time and $\mathrm{p} 2$ on a machine $B$ in discrete time. Then, in this instance a task consists in the resolution of both processes and can be represented as the term Task((S1, S2), (D1, D2)). The solution can be interpreted as follows: process $p 1$ has to begin its execution in machine A during the interval [3.75, 5.125] and, in this case, process $p 2$ has to start its execution, in machine $B$, during the interval $[4,5]$ (i.e., in the fourth or fifth unit of time in machine B). Alternatively, pl can begin its execution during the interval $[0.7,1.875]$ and, then, $p 2$ has to start during the interval $[1,2]$ (i.e., in the first or second unit of time in machine B). Solutions for each of the instances are graphically illustrated in Figure 7 where black dots mark the solution set.

\section{OPTIMIZATIONS}

This section discusses improvements that are being made to the $\mathrm{cl} p(\mathcal{L})$ system.

\subsection{Discrete Domains}

Suppose that $L$ is a discrete domain. Then we can identify equivalent elements of $L^{\mathrm{S}}$ and hencetheinterval domain $R_{\mathrm{L}}^{\mathrm{S}}$ by introducing the foll lowing equivalence 
PROGRAM :

into $(\operatorname{task}(S 1, D 1), \operatorname{task}(S 2, D 2)):-S 2<=: S 1, \operatorname{plus}(S 1, D 1, S D 1)$,

noDverlap (_, [] ).

plus (S2,D2,SD2), SD1 <=: SD2 .

noDverlap(task $(\mathrm{S} 1, \mathrm{D} 1),[\operatorname{task}(\mathrm{S} 2, \mathrm{D} 2) \mid$ Tasks] $)$ :-

((plus(S1,D1,SD1), SD1 <=: S2)

;

(plus(S2,D2,SD2), SD2 <=: S1)),

schedule([],_).

noDverlap(task (S1,D1), Tasks).

schedule([Task|Tasks], Supertask):- into(Task, Supertask), noDverlap (Task, Tasks),

schedule(Tasks, Supertask).

INSTANCE ON $\mathrm{clp}(\mathrm{FD})$

$\operatorname{clp}(\mathrm{L})>\operatorname{schedule}([\operatorname{task}(0,1), \operatorname{task}(3,1), \operatorname{task}(\mathrm{S}, 1)], \operatorname{task}(0,6))$.

solution: $S$ isin $(1$, close $) .(2$, close $)$ or $S$ isin $(4$, close $) \ldots(5, c l o s e)$.

INSTANCE ON clp(Real)

$\mathrm{clp}(\mathrm{L})>\operatorname{schedule}([\operatorname{task}(0.0,0.7), \operatorname{task}(2.75,1.0), \operatorname{task}(S, 0.875)], \operatorname{task}(0.0,6.0))$.

solution: $\mathrm{S}$ isin $(0.7, \mathrm{close}) \ldots(1.875, \mathrm{close})$ or $\mathrm{S}$ isin $(3.75, \mathrm{close}) \ldots(5.125, \mathrm{close})$

INSTANCE ON clp(Real $\times$ FD)

$\operatorname{clp}(\mathrm{L})>\operatorname{schedule}([\operatorname{task}((0.0,0),(0.7,1)), \operatorname{task}((2.75,3),(1.0,1)), \operatorname{task}(S,(0.875,1))]$, $\operatorname{task}((0.0,0),(6.0,6)))$.

solution: $S$ isin $((3.75,4)$, close $) \ldots((5.125,5)$, close $)$ or

$\mathrm{S}$ isin $((0.7,1)$, close $) \ldots((1.875,2)$, close $)$.

Fig. 6. An overloaded scheduling program.
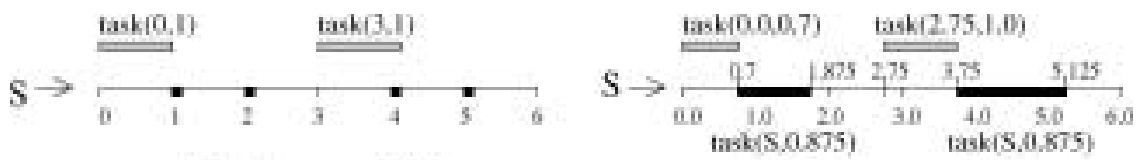

(a) Instanice on of $\mathrm{F}$ D )

(4) Instance on $\alpha+R$ J

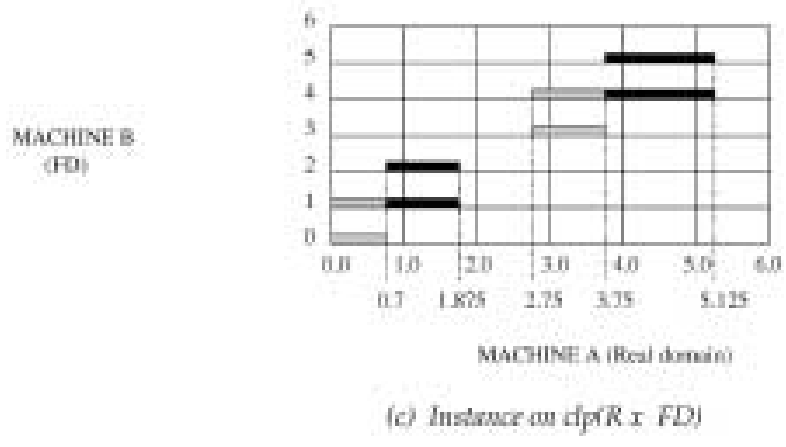

Fig. 7. Solving a scheduling problem.

ACM Transactions on Programming Languages and Systems, Vol. 26, No. 1, J anuary 2004. 
rule: for any $a \in \mathrm{L}$ for which the immediate predecessor pre(a) is defined and is unique,

$$
\text { a) } \equiv \text { pre(a) })_{1} \quad \text { in } L^{s} \text {. }
$$

By the duality principle of lattices we also have the dual rule: for any $a \in L$ for which the immediate successor $\operatorname{succ}(a)$ is defined and is unique,

$$
\overline{a)} \equiv \overline{\operatorname{succ}(a)_{]}} \text {in } \overline{L^{s}} \text {. }
$$

When the interval domain $L^{s}$ is constructed from a discrete domain $L$, then pre and succ provide a canonical form for $L^{s}$ and $\overline{L^{s}}$ (and hence for $R_{L}^{s}$ ) where the bracket ')' is eliminated in favor of the bracket ']'.

Example 14. For the Integer domain, pre(i) $=\mathbf{i}-1$ for any $\mathrm{i} \in \operatorname{Integer}$ so that, for instance, $3, \equiv 2_{\jmath}$ and $\left.\left\langle\overline{1_{1}}, 3\right\}\right\rangle \equiv\left\langle\overline{1_{1}}, 2_{\jmath}\right\rangle$. Suppose $L=\{0,1,2,3\}$ is a lattice where $0<1<2<3$. Then,

$$
\begin{aligned}
& \left.L^{S}=\left\{0_{1}, 0_{]}, 1_{)}, 1_{]}, 2_{2}, 2_{]}, 3\right), 3_{]}\right\} \equiv\left\{0,0_{]}, 1_{]}, 2_{]}, 3_{]}\right\}, \\
& \left.\left.\overline{L^{s}}=\{\overline{3}, \overline{3}, \overline{2}), \overline{2}\right], \overline{1_{1}}, \overline{1_{]}}, \overline{0_{1}}, \overline{0_{]}}\right\} \equiv\left\{\overline{3}, \overline{3}, \overline{2}, \overline{1_{]}}, \overline{0_{]}}\right\} \text {. }
\end{aligned}
$$

Similarly, with the Bool domain, pre(true) $=$ false so that true $\equiv$ false and $\langle\overline{\text { false }}$, true $\rangle \equiv\langle\overline{\text { false }}$, false $\rangle$.

With these rules for discrete domains, more inconsistencies can be detected.

Example 15. Consider again the domain $L$ in Example 6. Suppose $L$ is discrete and that $\operatorname{succ}(a)=c$ and $\operatorname{succ}(c)=b$. Then the ranges $\left.\langle\bar{a}), c_{)}\right\rangle \in R_{L}^{S}$ and $\left\langle\bar{c}, b_{j}\right\rangle$ are inconsistent since they are equivalent to ranges $\left\langle\bar{C}_{]}, a_{j}\right\rangle$ and $\left\langle\bar{b}_{]}, c_{j}\right\rangle$, respectively. These are the circled nodes in Figure 1.

\subsection{Nonlinear Constraints and Floating Point Arithmetic}

For the real domain, many constraint systems provide both a linear and a nonlinear solver. As the linear solver is the most efficient, this should be used whenever the constraints are linear. Although our solver does not provide direct support for solving nonlinear numeric equations, nonlinear constraints can be solved in $\mathrm{cl} p(\mathcal{L})$ by defining appropriate constraint operators. ${ }^{7}$

To see this, consider $\Re^{+}$, the domain of nonnegative reals, and a constraint such as ' $x * y=z^{\prime} / 3$. The main problems occur if it tries to evaluate $z / x$ when $x=0.0$ or $z / y$ when $y=0.0$. Of course, this constraint can be delayed until either $\mathrm{x}$ or $\mathrm{y}$ is ground and then check whether or not the ground term is 0.0 . However, more propagation can be obtained by defining ' $x * y=z$ ' $/ 3$ as a high-level constraint:

$$
\begin{aligned}
x * y=z \Leftrightarrow & x \sqsubseteq\langle\min (z) \pi \max (y), \max (z) / r \min (y)\rangle, \\
& y \sqsubseteq\langle\min (z) / \max (x), \max (z) / r \min (x)\rangle, \\
& z \sqsubseteq\langle\min (x) \overline{m i n}(y), \max (x) * \max (y)\rangle,
\end{aligned}
$$

\footnotetext{
${ }^{7}$ This is a generalization of the method proposed in Codognet and Diaz [1996a] for the clp(FD) system, and we use the same example in this paper.
} 
where $*, / 1, / r$ are declared in $\Re^{+s}$ as

$$
*:: \mathfrak{R}^{+s} \times \mathfrak{R}^{+s} \rightarrow \mathfrak{R}^{+s}, \quad / \quad:: \mathfrak{R}^{+s} \times \overline{\mathfrak{R}^{+s}} \rightarrow \mathfrak{R}^{+s}, \quad / \mathrm{r}:: \mathfrak{R}^{+s} \times \overline{\mathfrak{R}^{+s}} \rightarrow \mathfrak{R}^{+s} \text {, }
$$

and $/ 1, / \mathrm{r}$ in $\mathfrak{R}^{+}$and all the operators in $\mathrm{B}$ are defined as

$$
\begin{aligned}
a / 1 b & =a / b & \text { if } b \neq 0.0, \\
a / 1 b & =0.0 & \text { if } b=0.0, \\
a / r b & =a / b & \text { if } b \neq 0.0, \\
a / r b & =T_{\Re} & \text { if } b=0.0, \\
b_{1} \circ_{B} b_{2} & =\min _{B}\left(b_{1}, b_{2}\right) & \text { for } \circ \in\{*, / 1, / r\} .
\end{aligned}
$$

Observe that, as $b \neq 0.0$ is weaker than the condition $b$ is ground, there is more pruning than that obtained by delaying the evaluation of the constraint until it is linear.

Notice that this same proposal can be used to specify the rounding mode of floating point computations. For instance, a constraint such as ' $x^{2}=z^{\prime} / 2$ can be defined as

$$
\begin{aligned}
x^{2}=z \Leftrightarrow & x \sqsubseteq\left\langle\overline{\text { nearest }_{d}}(\overline{\operatorname{sqrt}}(\min (z))), \text { nearest }_{e}(\operatorname{sqrt}(\max (x)))\right\rangle, \\
& z \sqsubseteq\left\langle\overline{\text { nearest }_{d}}(\min (x) \mp \min (x)), \text { nearest }_{e}(\max (x) * \max (x))\right\rangle,
\end{aligned}
$$

where sqrt, nearest $t_{d}$, and nearest ${ }_{e}$ are declared on $\Re^{+s}$ as

$$
\text { sqrt }:: \mathfrak{R}^{+s} \rightarrow \mathfrak{R}^{+s} \text {, nearest } \mathrm{d}:: \mathfrak{R}^{+s} \rightarrow \mathfrak{R}^{+s}, \quad \text { nearest } \mathrm{e}:: \mathfrak{R}^{+s} \rightarrow \mathfrak{R}^{+s}
$$

and are defined in $\mathrm{B}$ as the identity and, for any $\mathrm{x} \in \mathfrak{R}^{+}$,

$$
\begin{aligned}
\operatorname{sqrt}(x) & \text { returns the square root of } x, \\
\text { nearest }_{d}(x) & \text { returns the nearest floating point number } \leq x, \\
\text { nearest }_{e}(x) & \text { returns the nearest floating point number } \geq x .
\end{aligned}
$$

This constraint will lead to better pruning than using the constraint ' $\mathrm{X} * \mathrm{X}=\mathrm{Z}$ '. Notethat the proposal in Benhamou et al. [1999] which combines the evaluation of primitive constraints with specific methods for solving nonlinear constraints is likely to be more efficient although less declarative.

\subsection{The precision/1 Map As a Normalization Rule}

When there exist more than one solution, some sort of domain splitting should be applied in order tolook for solutions in each of the resulting partitions of the problem. When the constraint system supports multiple domains, the precision map (see Definition 14) provides a useful way to normalize the heuristics for value ordering.

Example 16. The well-known first fail principle usually chooses the variable constrained by the smallest range. However, in systems supporting multiple domains, it is not always clear which is smallest. One way to compare the ranges is to use the map precision/1 defined for each computation domain. To see this, consider a set of variables $X=\left\{x_{1}, \ldots, x_{n}\right\}$ and constraint store 
$\mathrm{S}=\left\{\mathrm{C}_{1}, \ldots, \mathrm{c}_{\mathrm{n}}\right\} \in \mathcal{S S}^{\mathrm{X}}$ where for each $\mathrm{i} \in\{1, \ldots, \mathrm{n}\}, \mathrm{c}_{\mathrm{i}}$ is the simple interval constraint in $S$ with constrained variable $x_{i}$. Suppose that $S$ is divisible (i.e., $\mathrm{S}$ can be partitioned into, at least, two consistent stores). Then the first fail principle can be emulated with the procedure choose irstFail which chooses the "smallest" constraint in $\mathrm{S}$ that is divisible (i.e., one whose range can be partitioned into two consistent parts). ${ }^{8}$

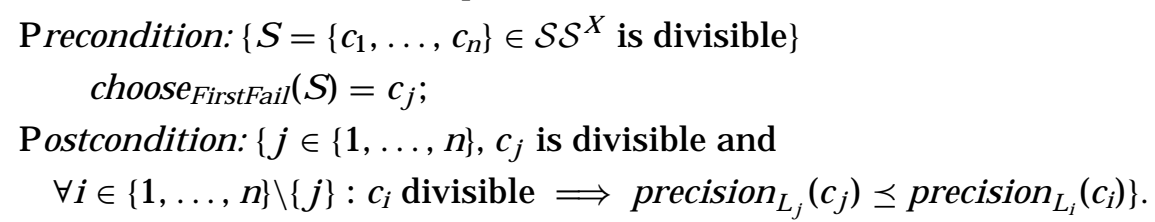

\subsection{Disjunctive Constraints}

It is well known that disjunctive constraints are sometimes useful for formulating a solution to a problem. In logic programming, one easy but inefficient way to handle disjunctive constraints is to create choice points. A more efficient approach that avoids the use of choice points would be to generalize the solution proposed by the clp(FD) system in Codognet and Diaz [1996a]. This is defined for the class of disjunctive constraints of the form $c_{1} \vee \cdots \vee c_{n}$ where each $c_{i}(1 \leq i \leq n)$ has the form $x_{1} \sqsubseteq r_{1}^{i} \wedge \cdots \wedge x_{k} \sqsubseteq r_{k}^{i}$ and is constrained on the same variables, that is, $\left\{x_{1}, \ldots, x_{k}\right\}$. The importance of this kind of disjunction is that, as observed in Codognet and Diaz [1996a], "nearly all current uses of constructive disjunction fit in this case." We can generalize this solution by incorporating, in the definition of the constraint $x \sqsubseteq r$, range union operations ${ }^{9}$ as $x \sqsubseteq r_{1} \vee \cdots \vee r_{n}$. This delays the creation of choice points and can lead to $a$ reduction in the size of the search tree.

An alternative technique, adapted from an idea shown in Van Hentenryck et al. [1998], would be to define the constructive disjunction, that is, to consider the range as an interval and the lub as defined for interval lattices [Slavík 1986]. Thus the lub of a set of ranges would be the range whose lower bound was the glb of the lower bounds and the upper bound, the lub of the upper bounds. However, this can lead to the addition of infeasible values. To see this, consider the domain Integer and let $r_{1}=\left\langle 1_{]}, 4_{]}\right\rangle$and $r_{2}=\left\langle 6_{]}, 10_{]}\right\rangle$. Then the range, $\mid \mathrm{ub}_{\mathcal{C}_{1}^{(v)}}\left(v \sqsubseteq r_{1}, v \sqsubseteq r_{2}\right)=v \sqsubseteq\left\langle 1_{]}, 10_{]}\right\rangle$, includes the value 5 which was not in either $r_{1}$ or $r_{2}$.

\subsection{Global Constraints}

Recently Hickey [2000] has shown that contraction algorithms (such as the Taylor contractor) can be implemented in a declarative style in the CLIP system. CLIP is a CLP(Interval( $\Re))$ system in which constraints are decomposed into sets of primitive constraints that are sent to a constraint-solving engine providing support for interval arithmetic and where computation is done over

\footnotetext{
${ }^{8}$ It is straightforward to include more conditions, for example, if $c_{i}, c_{k}, c_{j}$ have the same (minimum)

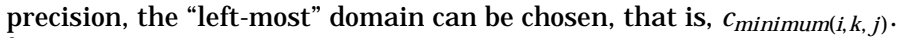

${ }^{9}$ In fact, $\vee$ is not the union operator but a composition operator since our ranges do not correspond to sets but to elements in our interval lattices.
} 
the floating point intervals associated to the constrained variables. We have al ready shown in Section 8.2, how our solver can handle floating point computations and haveshown how the (relational) product operator ' $\mathrm{x} * \mathrm{y}=\mathrm{z}$ '/3 for the floating point domain could be defined. In the same style, it is straightforward to define the relational floating point version of all the classical real interval operators, that is, $*,+,-$, and / [Moore 1966]. For example, the definition of the plus constraint in Example 13 corresponds to the classical definition of the + operator for interval arithmetic. It is straightforward to adapt this definition to the floating point real domain by using the operators nearest ${ }_{d}$ and nearest ${ }_{e}$ as done for the constraint ' $x * y=z$ '. Also, throughout the paper we have shown that the declarativenature of our solver allows the user to define (possibly cooperative, generic and/or overloaded) high-level constraints. As CLP(Interval( $($ ) is an instance of our framework, it is natural to expect that the CLIP approach for generating global contractors can also be adapted for our solver.

Note that, for the system domains (i.e., real, FD, set, or Bool ean domains), the implementation can provide specialized low-level optimizations. For example, for the real domain, well-known implementations of functions such as exp/1 or cos/1 can be used. Observe that these are now provided in our prototype implementation for $\mathrm{dl}(\mathcal{L})$ since these are already available in SICStus, the system in which our prototype is constructed.

\section{RELATED WORK}

Indexical-based implementations. The indexical approach from which our framework is derived was first implemented by Codognet and Diaz [1996a], where finite interval constraints of the form $\mathrm{x}$ in $\mathrm{r}$ were efficiently implemented using an extension of the WAM [Diaz and Codognet 1993]. Well-known CLP systems such as SICStus [Sicstus Manual 1994] and IF/Prolog [I f/Prolog 1994] now integrate the $x$ in $r$ constraint to provide a glass box solver for FD, the finite domain of integers.

In Codognet and Diaz [1993], the idea was extended to the clp(FD/B) system that integrates a Boolean solver into the existing FD solver. A version of the $\mathrm{clp}(\mathrm{FD} / \mathrm{B})$, called clp(B), devel oped by Codognet and Diaz [1994] solely for the Boolean domain, has an efficiency that was, on average, an order of magnitude faster than most of the existing Boolean solvers including, surprisingly, some special-purpose Boolean solvers.

More recently, Georget and Codognet [1998] used the indexical approach to implement a generic language for semiring-based constraint satisfaction (also on FD) and demonstrated its efficiency with respect to dedicated systems. Moreover, Goualard et al. [1999] described a system called DecLic that extends the $\mathrm{clp}(\mathrm{FD})$ solver to provide an efficient constraint solver for continuous domains (i.e., the real domain).

These indexical-based systems therefore show that the indexical approach can obtain competitive efficiency for the Boolean, finite, and continuous domains. Moreover, these systems demonstrate that the implementation of highlevel constraints (e.g., interval arithmetic) as rules (e.g., combination of simple primitive constraints) is fairly efficient. We therefore anticipate that we can 
adapt the techniques used for the implementation of $\mathrm{clp}(\mathrm{FD}), \mathrm{clp}(\mathrm{B})$, and DecL ic to our constraint system. Despite the expected loss of some optimizations for specific domains due to the generality of our framework, we expect to obtain reasonably competitive performance compared to domain-specific systems.

Other optimizations in the low level can be incorporated as done in the $\mathrm{clp}(\mathrm{FD})$ system. For example it is straightforward to incorporate the indexical $\operatorname{dom}(\mathrm{y})$ to return the whole range associated to $\mathrm{y}$. In this case a constraint such as $\mathrm{x} \sqsubseteq \operatorname{dom}(\mathrm{y})$ is equivalent to the constraint $\mathrm{x} \sqsubseteq\langle\min (\mathrm{y}), \max (\mathrm{y})\rangle$.

Interval reasoning. Interval arithmetic, on which the indexical approach to constraint solving is based, has been applied to constraint satisfaction problems over numeric domains [Benhamou 1995; Lee and van Emden 1993; Older 1989; Benhamou and Older 1997] and, in particular, to floating point numbers on relational programming [Cleary 1987]. In this latter application, interval computations are used to approximate a computed real number. This concept of approximation, which works well on numeric domains, does not generalize since it assumes that the closest value smaller (respectively higher) than any computed value is computable. To ensure that this property holds in our more generic framework, we have defined a new system of approximation that is applicable to any (possibly infinite) lattice.

Older and Vellino [1993] presented a lattice-theoretic semantics for numeric interval constraints that aims to capture the properties of both the primitive interval operations and the constraint propagation networks created from them. Based on lattice theory, some analogies with respect to our proposal can be detected: (1) the computation domain has a lattice structure and is constructed from the bounds of the intervals; (2) the theory can be applied with infinite precision (i.e., without approximating a real to a floating point number) although only on reals; (3) the operators are assumed to maintain properties over the computation domain that are also maintained by our constraint operators (e.g., monotonicity); (4) the propagation process is based on a fixed-point semantics. In spite of the similarities, there are a number of aspects that made this approach very different from our proposal: (1) the framework is developed exclusively for numeric domains; (2) we provide a control mechanism, at the user level, for the propagation by allowing the constraint operators to be defined directly on the bounds of the interval; in this sense, Older and Vellino [1993] did not treat the issue of the transparency of their theory; (3) the theory proposed in Older and Vellino [1993] is "quite abstract and ther efore somewhat remote from actual implementations," whereas our theory can be directly implemented; (4) we treat the termination issue even for nonnumeric domains; (5) solver cooperation was not treated at all in Older and Vellino [1993] and (6) implementation issues were neglected.

Generic constraint solving procedures. Apt [1999, 2000] proposed a framework for constraint propagation based on chaotic iteration algorithms for partially ordered domains. A key observation in these papers was that most constraint propagation algorithms presented in the literature can be expressed as direct instances of these algorithms. There are many similarities between 
the frameworks described here and in Apt [1999] and these, together with the main differences, were discussed in detail in Fernández and Hill [1999a], where we showed that the process of constraint propagation in our operational schema can be viewed as a process of function evaluation in the chaoticiteration algorithm given in Apt [1999]. One difference is that, in Apt [1999], the chaotic iteration approach was specialized for a constraint satisfaction problem where the set of constraints to be solved was interpreted as sets of possible solutions whereas a set of interval constraints in our framework embodies more: the intended interaction between the variables in the constraint propagation. A second important difference is that the chaotic iteration approach assumes the finite chain property ${ }^{10}$ whereas our domains do not necessarily possess this property. Further study on how the idea of computing an approximate solution via a precision map as defined in this paper can be adapted for a chaotic iteration algorithm is needed. Note that a technique such as this could be useful in extending the framework described in Apt [2000] to domains not satisfying the finite chain property.

General frameworks for solving soft constraints (i.e., constraints with an associated confidencevalue, for example, cost, uncertainty, or degree) have been described by both Schiex et al . [1995] and Bistarelli et al. [1995]. The framework in Schiex et al. [1995] was defined for any finite totally ordered domain which has a specific operation satisfying certain properties whereas the framework in Bistarelli et al. [1995], was defined for a finite semiring structure. In neither case was the domain allowed to be infinite.

A genericform of constraint propagation called general ized propagation (GP), proposed in [Le Provost and Wallace 1993], is applicable to arbitrary computation domains. Note that, unlike our proposal, the constraints are not defined generically but use any available constraint over any computation domain to express restrictions on problem variables. One drawback of GP compared to our approach, is that termination of the search for answers to a propagation constraint is not guaranteed and the entire responsibility for ensuring termination remains with the programmer.

Solver communi cati on and cooperation. Baader and Schulz [1995] provided an abstract framework for combining different and (unlike in our proposal) independently defined constraint languages and constraint solvers. Thus, they were primarily concerned with the properties that such a combined solution structure should satisfy.

A general scheme for solver cooperation was proposed by Hofstedt [2000]. In this paper, domains were defined by using " $\Sigma$-Structures" in a sorted language and a constraint was a relation over an n-ary Cartesian product of the domains. As for our framework, solvers are combined by means of the Cartesian product of the domains. However, H ofstedt [2000] assumed that each component domain has its own associated solver built into the system and therefore focused on the interface between the component solvers; the complete system consisted of the

\footnotetext{
${ }^{10} A$ domain $L$ satisfies the finite chain property if every increasing sequence $a_{0} \leq a_{1} \leq a_{2} \ldots$ of its elements eventually stabilises, that is, for some $j>0, a_{i}=a$ for $i \geq j$ and $a \in L$.
} 
interface plus a set of built-in constraint systems. In contrast, in our proposal the high-level constraints determine the possible cooperation that can occur between the domains and their solvers and these constraints may be defined by the user or system. Note that the flexibility of these high-level constraints implies that the solver interface defined by Hofstedt [2000] could be implemented in our system.

Constraint systems such as CLP(BNR) [Benhamou and Older 1997] and Prolog IV [N'Dong 1997] provide some support for cooperation between solvers. However, in these languages, the solver cooperation is mainly limited to Booleans, reals, naturals lists, and trees. Moreover, this cooperation is usually hard-wired and built into the language.

Interval lattice theory. The interval topology which is the lattice of closed intervals of a lattice [Birkhoff 1967] appears to be similar to the intervals on which our constraints are based and it would have been useful if we could have based our interval constraints on this formalism. However, in our framework, to allow for approximations in the continuous domains, the intervals are not necessarily closed, so that the ranges for a domain $L \in \mathcal{L}$ are not necessarily meet- or join-complete sublattices of $L$ and do not form an interval topology. Second, in order to guaranteethemonotonicity of our interval constraints (using theindexical functions $\min / 1, \overline{\mathrm{val}} / 1$ and $\max / 1, \mathrm{val} / 1$ ) and identify, prior to the resolution step, those constraints that do not lead to further propagation, we needed to distinguish between a domain and its mirror.

\section{CONCLUSIONS}

We have defined a framework for constraint solving over lattices and illustrated with many examples the versatility and expressivity of this approach. For maximum generality and to allow for any lattice, finite or infinite, discrete or continuous, we have constructed the interval domains in several stages, each stage taking advantage of the lattice structures inherited from the underlying computation domains on which the interval domains are built. Thus, we first defined and added the bracket domain $B$ to each computation domain $L$ to create the right bounded domain $\mathrm{L}^{\mathrm{S}}$ for open and closed (right) bounds for the intervals. We then defined the symmetric mirror domain $\overline{L^{s}}$ so as to allow for the left bounds of intervals. These bounds were then combined using the direct product of lattices to form the range elements of the interval domain $\mathrm{R}_{\mathrm{L}}^{\mathrm{s}}$. Finally, we added the variable to be constrained to the given range to form the interval constraint $x \sqsubseteq r$.

When defining theelements of the bounded computation domain $L^{b}$, weintroduced two additional constructs. One, which generalizes an idea from Codognet and Diaz [1996a], was indexicals max(x), val(x), for the right bounded domain and $\min (x), \overline{\operatorname{val}(x)}$, for the left bounded domain. These provide necessary links between the ranges for the constrained variables and give the user transparent control over the constraint propagation. The other was that of an operator oL which maps a domain constructed from several, possibly distinct, computation domains $L_{1}, \ldots, L_{n}$ to another, possibly different, codomain $L$. This, combined with the indexicals, allows a one-way communication from the domains 
$L_{1}^{b}, \ldots, L_{n}^{b}$ to the domain $L^{b}$. Finally, for full solver cooperation, we have shown how a high-level constraint defined as a relation over a domain constructed from a set of computation domains can provide unrestricted communication between these domains. Notice that this formalization of the framework is new even when restricted to just the finite domains of integers.

We have presented an operational schema for solving these constraints and proved it correct. In the case of the nonfinite domains, termination of the procedure can only be guaranteed by letting the solver return an approximation to the correct result. An idea from Sidebottom and Havens [1992] for controlling accuracy in the processing of disjoint intervals over the reals was adapted for our lattice domains. The special operator precisi on ${ }_{L} / 1$ that maps the domain elements to nonnegative reals $\Re^{+}$and a limit element $\varepsilon \in \Re^{+}$that controls the degree of the approximation were introduced. Observe that the notion of our precision operator corresponds, in some sense, to a change of domain where the domains (i.e., intervals) which are "too small" but still consistent are not considered in the lattice. The basic operational schema was then adapted so as to check, using these precision and limit constructs, for just an approximation to the fix-point. With this modification of the schema, we proved that such a procedure terminates with an approximate solution.

Observethat the framework, being applicableto any lattice, provides support for all the existing practical domains in CLP (e.g., reals, integers, sets, and Booleans). Mor eover, by using lattice combinators, new compound domains and their solvers can easily be obtained from previously defined domains such as these. We have imposed the restriction that the sets of constrained variables associated to each computation must be disjoint. However, it should be possible to remove such a restriction and consider sublattices of lattices as computation domains. These could provide a means of having variable sets of a sublattice being also allowed as variables in the main lattice. This is another topic for future work.

To demonstrate our framework is realizable in a practical setting, we have devel oped the CLP language $\mathrm{cl}(\mathcal{L})$ and built a prototype implementation using CHR's [Frühwirth 1998]. This prototype supports a set of built-in domains as well as user-defined domains. N ote that all the examples in this paper have been solved with this implementation of $\operatorname{clp}(\mathcal{L})$. We note that this implementation is only a prototype and it does not compete with the CHRs although it may be considered as a CHR module. In fact, this system shows the feasibility of our ideas and was not designed with efficiency in mind. Thus the development of an efficient implementation is future work.

\section{APPENDIX A. PROOFS}

Remark 1. As in the main part of the paper, $L \in \mathcal{L}, V_{L}$ is the set of variables associated with $\mathrm{L}, \mathcal{V}_{\mathcal{L}}=\cup\left\{\mathrm{V}_{\mathrm{L}} \mid \mathrm{L} \in \mathcal{L}\right\}, \mathrm{R}_{\mathrm{L}}^{\mathrm{b}}$ is the interval domain over $\mathrm{L}, \mathrm{X} \in$ $\wp_{\mathrm{f}}\left(\mathcal{V}_{\mathcal{L}}\right)$ is the set of constrained variables, $\mathcal{C}^{\mathrm{X}}$ is the set of all interval constraints for $\mathrm{X}$, and $\mathcal{S S}^{\mathrm{X}}$ is the set of all simple stable constraint stores for $\mathrm{X}$. In the proofs, elements of the bounded computation domain are denoted $a s a_{b}$ or $(a, b)$, depending on context. 
Proposition 3. Let $L^{\prime} \in\left\{L^{s}, \overline{L^{s}}\right\}$ and $t \in L^{\prime}$. Then,

$$
\text { (1) } \overline{\overline{L^{\prime}}}=L^{\prime}, \quad \text { (2) } \overline{\mathrm{E}}=\mathrm{t} \text {. }
$$

Proof. We prove the cases separately.

(1) Observe that

$$
\overline{\overline{L^{s}}}={ }^{1} \overline{\overline{(L, B)}}={ }^{1}(\hat{\hat{L}}, B)={ }^{2}(L, B)={ }^{1} L^{s},
$$

where equality, $={ }^{1}$ follows from Definition 2 , and $={ }^{2}$ foll ows from the duality definition for lattices. Thus, if $L^{\prime}=L^{s}$, then the result follows. Moreover, if $\mathrm{L}^{\prime}=\overline{\mathrm{L}^{\mathrm{s}}}$, then $\overline{\overline{\mathrm{L}^{\prime}}}=\overline{\overline{\overline{\mathrm{L}^{s}}}}=\overline{\overline{\mathrm{L}^{s}}}=\mathrm{L}^{\prime}$.

(2) Observe that, for some $a \in L \cup \hat{L}$ and $t=a_{b} \in\left\{L^{s}, \overline{L^{s}}\right\}$, we have

$$
\overline{\mathrm{t}}=\overline{\overline{\mathrm{a}_{\mathrm{b}}}}={ }^{1} \overline{(\hat{\mathrm{a}}, \mathrm{b})}={ }^{1}(\hat{\mathrm{a}}, \mathrm{b})={ }^{2}(\mathrm{a}, \mathrm{b})={ }^{1} \mathrm{a}_{\mathrm{b}}=\mathrm{t},
$$

where equality $={ }^{1}$ follows from Definition 2 and $={ }^{2}$ from the duality definition for lattices.

Proposition 4. Suppose $\circ$ is a constraint operator for $L^{s}$. Then,

o is monotonic;

$\overline{0}$ is a constraint operator (for $\overline{L^{s}}$ );

$\circ$ is the mirror of $\bar{o}$ i.e, $\circ \equiv \overline{\bar{o}}$.

Proof. Suppose that $\circ:: L_{1}^{s} \times \cdots \times L_{n}^{s} \rightarrow L^{s}$ is a constraint operator. We prove the cases separately.

(a) Suppose $t_{i} \preceq t_{i}^{\prime}$ for $i \in\{1, \ldots, n\}$, where $t_{i}=\left(a_{i}, b_{i}\right)$ and $t_{i}^{\prime}=\left(a_{i}^{\prime}, b_{i}^{\prime}\right)$. We need to show

Observe that

$$
\circ\left(t_{1}, \ldots, t_{n}\right) \preceq \circ\left(t_{1}^{\prime}, \ldots, t_{n}^{\prime}\right) .
$$

by the product of lattices: $a_{i} \preceq a_{i}^{\prime}$; moreover if $a_{i}=a_{i}^{\prime}$ then $b_{1} \preceq b_{i}^{\prime}$;

by monotonicity of $\circ_{L}: \quad o_{L}\left(a_{1}, \ldots, a_{n}\right) \preceq \circ_{L}\left(a_{1}^{\prime}, \ldots, a_{n}^{\prime}\right)$.

If $o_{L}\left(a_{1}, \ldots, a_{n}\right) \prec o_{L}\left(a_{1}^{\prime}, \ldots, a_{n}^{\prime}\right)$ then (5) holds by the product of lattices and Definition 3.

Otherwise, $o_{L}\left(a_{1}, \ldots, a_{n}\right)=o_{L}\left(a_{1}^{\prime}, \ldots, a_{n}^{\prime}\right)$. There are two cases:

(1) $\forall i \in\{1, \ldots, n\}, a_{i}=a_{i}^{\prime}$. Then, by monotonicity of $\circ_{B}$,

$$
\circ_{B}\left(b_{1}, \ldots, b_{n}\right) \preceq \circ_{B}\left(b_{1}^{\prime}, \ldots, b_{n}^{\prime}\right) \text {. }
$$

(2) $\exists i \in\{1, \ldots, n\}, a_{i} \prec a_{i}^{\prime}$. Then $\circ L$ is not a strict monotonic function and, by Definition $3, \circ_{B}$ is a constant, that is, $\circ_{B}\left(b_{1}, \ldots, b_{n}\right)={ }_{{ }_{B}}\left(b_{1}^{\prime}, \ldots, b_{n}^{\prime}\right)$.

Thus, in both cases, (5) holds by the product of lattices and Definition 3.

(b) Observe that

$$
\begin{array}{ll}
\bar{\sigma}:: \overline{L_{1}^{s}} \times \cdots \times \overline{L_{n}^{s}} \rightarrow \overline{L^{s}} & \text { (by Definition 3), } \\
\bar{\sigma}::{\widehat{L_{1}^{s}}}^{s} \times \cdots \times{\widehat{L_{n}^{s}}}^{s} \rightarrow \hat{L}^{s} & \text { (by Definition 2), }
\end{array}
$$

where, for $i \in\{1, \ldots, n\}, \hat{L_{i}} \in \mathcal{L} \cup \hat{\mathcal{L}}$ and $\hat{L} \in \hat{\mathcal{L}}$. 
Assuming the notation of Definition 3 , we have, if $t_{i}=\left(a_{i}, b_{i}\right)(1 \leq i \leq n)$, then $\circ\left(t_{1}, \ldots, t_{n}\right)=\circ_{L}\left(a_{1}, \ldots, a_{n}\right)_{o_{B}}\left(b_{1}, \ldots, b_{n}\right)$.

Let $o_{L}:: \widehat{L}_{1} \times \cdots \times \widehat{L_{n}} \rightarrow \hat{L}$ be the dual operator to $o_{L}$ so that, if ${ }_{\mathrm{L}}\left(\mathrm{a}_{1}, \ldots, \mathrm{a}_{\mathrm{n}}\right)=\mathrm{a}$, then ${ }_{\mathrm{L}}\left(\hat{a_{1}}, \ldots, \hat{a_{n}}\right)=\hat{a}$. Then, by the duality principle of lattices, $o_{\hat{L}}$ is monotonic in $\hat{L}$. Moreover, $o_{L}$ is strict monotonic whenever ${ }_{\mathrm{L}} \hat{\mathrm{L}}$ is. Hence, we have

$$
\begin{aligned}
& \bar{o}\left(\bar{t}_{1}, \ldots, \bar{t}_{n}\right)=\overline{o\left(t_{1}, \ldots, t_{n}\right)} \\
& \text { (by Definition 3) } \\
& =\overline{O_{L}\left(a_{1}, \ldots, a_{n}\right)_{O_{B}}\left(b_{1}, \ldots, b_{n}\right)} \\
& \text { (by Definition 3) } \\
& =\left(\circ_{L}\left(\widehat{a_{1}, \ldots}, a_{n}\right), \circ_{B}\left(b_{1}, \ldots, b_{n}\right)\right) \\
& \text { (by Definition 2) } \\
& =\left(o_{\hat{L}}\left(\widehat{a_{1}}, \ldots, \widehat{a_{n}}\right), \circ_{B}\left(b_{1}, \ldots, b_{n}\right)\right) \\
& =\sigma_{\hat{L}}\left(\widehat{a_{1}}, \ldots, \widehat{a_{n}}\right)_{o_{B}}\left(b_{1}, \ldots, b_{n}\right) \\
& \text { (by the definition of } O_{\hat{L}} \text { ) } \\
& \text { (by Definition 3). }
\end{aligned}
$$

Thus $\bar{\sigma}$ is a constraint operator (for $\overline{L s}$ ) as defined in Definition 3.

(c) Observe that

$$
\begin{array}{lr}
\overline{\mathrm{o}}:: \overline{\mathrm{L}_{1}^{s}} \times \cdots \times \overline{\mathrm{L}_{n}^{s}} \rightarrow \overline{\mathrm{Ls}^{s}} & \text { (by Definition 3); } \\
\overline{\bar{o}}:: \overline{\overline{\mathrm{L}_{1}^{s}}} \times \cdots \times \overline{\overline{\mathrm{L}_{n}^{s}}} \rightarrow \overline{\overline{\mathrm{Ls}^{s}}} & \text { (by Definition 3); } \\
\overline{\bar{\sigma}}:: \mathrm{L}_{1}^{s} \times \cdots \times \mathrm{L}_{n}^{s} \rightarrow \mathrm{L}^{s} & \text { (by Proposition 3(1)). }
\end{array}
$$

Also if $t_{i} \in L_{i}^{s}$ for all $i \in\{1, \ldots, n\}$,

$$
\begin{aligned}
& \overline{\bar{o}}\left(t_{1}, \ldots, t_{n}\right)=\overline{\bar{o}}\left(\overline{\bar{t}_{1}}, \ldots, \overline{\mathrm{t}_{n}}\right) \\
& =\overline{\overline{o\left(t_{1}, \ldots, t_{n}\right)}} \\
& =\circ\left(t_{1}, \ldots, t_{n}\right)
\end{aligned}
$$

(by Proposition 3(2))

(by Definition 3, applied twice)

(by Proposition 3(2)).

Therefore $\overline{\bar{o}}$ is equivalent to $\circ$.

Proposition 1. (See Section 3.4.)

Proof. Suppose that

$$
\begin{array}{ll}
r=\langle\bar{s}, t\rangle, & r^{\prime}=\left\langle\overline{s^{\prime}}, t^{\prime}\right\rangle, \\
s=\left(a, b_{1}\right), & s^{\prime}=\left(a^{\prime}, b_{1}^{\prime}\right), \\
t=\left(c, b_{2}\right), & t^{\prime}=\left(c^{\prime}, b_{2}^{\prime}\right) .
\end{array}
$$

By hypothesis, $r \preceq r^{\prime}$ and, by Definition 5 and by the product of lattices (i.e., direct product),

$$
\begin{aligned}
& \overline{\mathrm{s}} \preceq \overline{\mathrm{S}^{\prime}} ; \\
& \mathrm{t} \preceq \mathrm{t}^{\prime} .
\end{aligned}
$$

From (6):

$$
\begin{aligned}
\bar{s} \preceq \overline{\mathrm{s}^{\prime}} & \Rightarrow^{1}\left(\hat{\mathrm{a}}, \mathrm{b}_{1}\right) \preceq\left(\hat{a^{\prime}}, \mathrm{b}_{1}^{\prime}\right) \\
& \Rightarrow^{2} \hat{\mathrm{a}} \prec \hat{\mathrm{a}^{\prime}} \text { or } \hat{\mathrm{a}}=\hat{\mathrm{a}^{\prime}} \text { and } \mathrm{b}_{1} \preceq \mathrm{b}_{1}^{\prime} \\
& \left.\left.\Rightarrow^{3} \mathrm{a}^{\prime} \prec \mathrm{a} \text { or } \mathrm{a}=\mathrm{a}^{\prime} \text { and }\right\}_{1} \preceq\right\}_{1}^{\prime} \\
& \Rightarrow^{2}\left\{\begin{array}{l}
\text { if } \mathrm{a} \prec \mathrm{a}^{\prime} \text { then } \mathrm{s}^{\prime} \prec \mathrm{s} \\
\text { if } \mathrm{a}=\mathrm{a}^{\prime} \text { then } \mathrm{s} \preceq \mathrm{s}^{\prime},
\end{array}\right.
\end{aligned}
$$


where $\Rightarrow^{1}$ follows from Definition 2, where $\Rightarrow^{2}$ follows from the product of lattices (i.e., the lexicographic product) and $\Rightarrow^{3}$ follows from the duality principle for lattices in Section 2.

We suppose that $r^{\prime}$ is inconsistent. Then, by Definition 6 , we have three cases:

$$
\begin{aligned}
& \mathrm{t}^{\prime} \prec \mathrm{s}^{\prime} ; \\
& \mathrm{s}^{\prime} \nsim \mathrm{t}^{\prime} ; \\
& \left.\left.\mathrm{s}^{\prime}=\mathrm{a}^{\prime}\right) \text { and } \mathrm{t}^{\prime}={ }^{\prime} \mathrm{a}\right\}_{2}^{\prime} .
\end{aligned}
$$

In the following:

$\Rightarrow^{4}$ follows from Equation (8);

$\Rightarrow^{5}$ follows from (7);

$\Rightarrow{ }^{6}$ follows from Definition 6 ;

$\Rightarrow^{7}$ follows from Definition 2;

$\Rightarrow^{8}$ follows from the product of lattices (i.e., the lexicographic product);

$\Rightarrow{ }^{9}$ follows from a contradiction;

$\Rightarrow^{10}$ follows from Case (i) (i.e., $\mathrm{t}^{\prime} \prec \mathrm{s}^{\prime}$ );

$\Rightarrow^{11}$ follows from (7) since $\mathrm{t} \preceq \mathrm{t}^{\prime}$ and, by the product of lattices (i.e., lexicographic product), $\mathrm{C} \preceq \mathrm{C}^{\prime}$;

$\Rightarrow{ }^{12}$ follows from Case (ii) so that $\mathrm{s}^{\prime} \nsim \mathrm{t}^{\prime}$. Then, by Definition 2 and by the product of lattices (i.e., lexicographic product), $a^{\prime} \chi_{L} C^{\prime}$ and thus a $\varkappa_{L} C^{\prime}$.

As shown in (8), $a \prec a^{\prime}$ or $a=a^{\prime}$. Suppose first $a \prec a^{\prime}$. Then,

$$
\begin{aligned}
& \text { if (i) } \Rightarrow^{5} \mathrm{t} \prec \mathrm{s}^{\prime} \Rightarrow^{4} \mathrm{t} \prec \mathrm{s} \Rightarrow^{6} \mathrm{r} \text { is inconsistent; } \\
& \text { else if (ii) } \Rightarrow^{4} \mathrm{~s} \nsucc \mathrm{t}^{\prime} \Rightarrow^{5} \mathrm{~s} \nsim \mathrm{t} \Rightarrow^{6} \mathrm{r} \text { is inconsistent; } \\
& \text { otherwise if (iii) } \Rightarrow^{8} \mathrm{~s} \prec \mathrm{s}^{\prime} \Rightarrow^{4} \text { false. }
\end{aligned}
$$

Suppose now $a=a^{\prime}$. Then,

if (i) $\Rightarrow^{4} \mathrm{~s} \preceq \mathrm{s}^{\prime} \Rightarrow\left\{\begin{array}{r}\mathrm{s}=\mathrm{s}^{\prime} \Rightarrow^{10} \mathrm{t}^{\prime} \prec \mathrm{s} \Rightarrow^{5} \mathrm{t} \prec \mathrm{s} \Rightarrow^{6} \mathrm{r} \text { is inconsistent; } \\ \left.\left.\left.\mathrm{s} \prec \mathrm{s}^{\prime} \Rightarrow^{7} \mathrm{~s}=\mathrm{a}\right) \text { and } \mathrm{s}^{\prime}=\mathrm{a}\right] \Rightarrow^{10} \mathrm{t}^{\prime} \prec \mathrm{a}\right] \\ \left.\left.\quad \Rightarrow^{8} \mathrm{t}^{\prime} \preceq \mathrm{a}\right) \Rightarrow^{5} \mathrm{t} \preceq \mathrm{a}\right) \Rightarrow^{6} \mathrm{r} \text { is inconsistent; }\end{array}\right.$

else if (ii) $\Rightarrow^{4} \mathrm{~s} \nsucc \mathrm{t}^{\prime} \Rightarrow^{5} \mathrm{~s} \nsim \mathrm{t} \Rightarrow^{6} \mathrm{r}$ is inconsistent;

otherwise if (iii) $\left.\Rightarrow^{4} \mathrm{~s}=\mathrm{a}^{\prime}\right) \Rightarrow^{5},\left\{\begin{array}{c}\mathrm{t}=\mathrm{t}^{\prime} \Rightarrow \mathrm{t}=\left(\mathrm{a}^{\prime}, \mathrm{b}_{2}^{\prime}\right) \Rightarrow^{6} \mathrm{r} \text { is inconsistent; } \\ \mathrm{t} \prec \mathrm{t}^{\prime}\left\{\begin{array}{c}\left.\Rightarrow^{8} \mathrm{c}=\mathrm{a}^{\prime} \text { and } \mathrm{b}_{2} \preceq \mathrm{b}_{2}^{\prime} \Rightarrow \mathrm{t}=\mathrm{a}^{\prime}\right\}_{2} \\ \Rightarrow^{6} \mathrm{r} \text { is inconsistent; } \\ \Rightarrow^{8} \mathrm{c} \prec \mathrm{a}^{\prime} \Rightarrow^{8} \mathrm{t} \prec \mathrm{s} \Rightarrow^{6} \mathrm{r} \text { is inconsistent. }\end{array}\right.\end{array}\right.$

Thus, in all cases, $r$ is inconsistent.

Proposition 5. Let $\mathrm{S}, \mathrm{S}^{\prime} \in \mathcal{S S}^{\mathrm{X}}$ and let also $\mathrm{X}^{\prime} \subseteq \mathrm{X}$ and $\mathrm{C} \in \mathcal{S}^{\mathrm{X}^{\prime}}$. Then, if $\mathrm{S} \cup \mathrm{C} \mapsto \mathrm{S}^{\prime}, \mathrm{S}^{\prime} \preceq \mathrm{S}$. 
Proof. Suppose for each $x \in X, x$ is constrained by the constraints $c_{x} \in S$ and $c_{x}^{\prime} \in S^{\prime}$. Also, if $x \in X^{\prime}$, suppose that $C_{x}$ is the set of constraints in $C$ with constrained variable $\mathrm{x}$.

Then, by Definition 12,

$$
\begin{array}{ll}
C_{x}^{\prime}=\cap_{L}\left(C_{x} \cup\left\{C_{x}\right\}\right) & \text { if } x \in X^{\prime}, \\
C_{x}^{\prime}=C_{x} & \text { otherwise. }
\end{array}
$$

Therefore, by Definition 8 and the resulting contractance property, $c_{x}^{\prime} \preceq c_{x}$ for each $x \in X$. As consequence, by Definition $9, S^{\prime} \preceq S$.

Proposition 6. Suppose $S, \mathrm{~S}^{\prime} \in \mathcal{S S}^{\mathrm{X}}$ where $\mathrm{S} \preceq \mathrm{S}^{\prime}$. Then, if $\mathrm{S}^{\prime}$ is inconsistent, $\mathrm{S}$ is al so inconsistent.

Proof. Suppose that $S^{\prime}$ is inconsistent. Then, by Definition 9, there is, at least, one inconsistent constraint $c_{x}^{\prime}=x \sqsubseteq r^{\prime} \in S^{\prime}$ (for some $x \in X$ ). By Definition 7, this means that $r^{\prime}$ is inconsistent.

Let $c_{x}=x \sqsubseteq r$ be the constraint for $x$ in $S$. By hypothesis, $S \preceq S^{\prime}$ so that by Definition $9, c_{x} \preceq c_{x}^{\prime}$ for all $x \in X$, and by Definition $7, r \preceq r^{\prime}$. Thus, by Proposition $1, r$ is also inconsistent. Thus, by Definition $7, c_{x}$ is also inconsistent and hence, by Definition $9, \mathrm{~S}$ is inconsistent.

Lemma 1. Suppose that $c \preceq C^{\prime}$ are simple consistent constraints for $L \in \mathcal{L}$ constraining the same variable $\mathrm{y} \in \mathrm{X}$ and suppose also that $\mathrm{c}^{\prime}=\mathrm{y} \sqsubseteq\left\langle\overline{\mathrm{t}^{\prime}}, \mathrm{t}^{\prime}\right\rangle$ for somet $t^{\prime} \in \mathrm{L}^{\mathrm{s}}$. Then $\mathrm{c}=\mathrm{C}^{\prime}$.

Proof. Suppose that $t^{\prime}=a_{b}$, for some $a \in L$. Then, as $C^{\prime}$ is consistent, by Definition $6, \mathrm{t}^{\prime} \neq \mathrm{a}$ ) so that $\mathrm{t}^{\prime}=\mathrm{a}_{\mathrm{j}}$. Suppose also that $\mathrm{c}=\mathrm{y} \sqsubseteq\langle\overline{\mathrm{s}}, \mathrm{t}\rangle$. Then, by Definition $7,\langle\bar{s}, t\rangle \preceq\left\langle\overline{a_{1}}, a_{]}\right\rangle$, and, by Definition 5 ,

$$
\overline{\mathrm{s}} \preceq \overline{\mathrm{a}} \text { and } \mathrm{t} \preceq \mathrm{a}_{\text {] }} \text {. }
$$

Then, by Definition 2 and by the product of Iattices (i.e., the lexicographic product),

$$
\left(\bar{s}=\overline{a_{j}} \text { or } \bar{s}=\bar{a} \text { ) or } \bar{s}=\overline{a_{b^{\prime}}} \text { and } \widehat{a_{1}} \prec \hat{a}\right)
$$

and

$$
\left(t=a_{\text {j }} \text { or } t=a \text { ) or } t=a_{2_{b^{\prime}}} \text { and } a_{2} \prec a\right) \text {. }
$$

By the duality principle of lattices in Section 2 , this is equivalent to

$$
\left(s=a_{\text {] }} \text { or } s=a_{\text {) }} \text { or } s=a_{1^{\prime}} \text { and } a \prec a_{1}\right)
$$

and

$$
\left(\mathrm{t}=\mathrm{a}_{\text {] }} \text { or } \mathrm{t}=\mathrm{a} \text { ) or } \mathrm{t}=\mathrm{a}_{\mathrm{b}^{\prime}} \text {, and } \mathrm{a}_{2} \prec \mathrm{a}_{\text {. }}\right. \text {. }
$$

However, $\mathrm{c}$ is consistent so that, by Definition $7,\langle\bar{s}, \mathrm{t}\rangle$ is consistent. By Definition 6 , this means that $s \preceq t$ and, if $s=a$ ) then $t \neq a_{\text {] }}$. The only case for which this holds is when $s=t=a_{\text {] }}$.

Lemma 2. Let $\mathrm{S}_{1}, \mathrm{~S}_{2} \in \mathcal{S S}^{\mathrm{X}}$ be two consistent stores such that $\mathrm{S}_{1} \preceq \mathrm{S}_{2}$ and $C, c_{2} \in \mathcal{C}^{X}$ such that $C_{\rightsquigarrow} S^{2} C_{2}$. Then, there exists $C_{1} \in \mathcal{C}^{X}$ such that $C_{\rightsquigarrow}{ }^{S_{1}} C_{1}$ and $\mathrm{C}_{1} \preceq \mathrm{C}_{2}$. 
Proof. Let $\mathrm{C}=\mathrm{x} \sqsubseteq\langle\overline{\mathrm{S}}, \mathrm{t}\rangle$ where $\mathrm{x} \in \mathrm{X}$ and $\mathrm{x} \in \mathrm{V}_{\mathrm{L}}$ for some $\mathrm{L} \in \mathcal{L}$. Then as $\mathrm{C} \rightsquigarrow \mathrm{S}_{2} \mathrm{C}_{2}$, by Definition $11, \mathrm{C}_{2}=\operatorname{eval}\left(\mathrm{S}_{2}, \mathrm{c}\right)$ and $\mathrm{c}_{2}$ is simple. Then it follows from the Definition 10 and Definition 7 that

$$
\begin{gathered}
\mathrm{C}_{2}=\mathrm{x} \sqsubseteq\left\langle\operatorname{eval}\left(\mathrm{S}_{2}, \bar{s}\right) \text {, eval }\left(\mathrm{S}_{2}, \mathrm{t}\right)\right\rangle, \\
\operatorname{eval}\left(\mathrm{S}_{2}, \bar{s}\right) \in \overline{\mathrm{L}^{\mathrm{s}}} \text { and } \operatorname{eval}\left(\mathrm{S}_{2}, \mathrm{t}\right) \in \mathrm{L}^{\mathrm{s}} .
\end{gathered}
$$

Suppose that $c_{1}=x \sqsubseteq \operatorname{eval}\left(S_{1}, c\right)$. Then, again, it follows from Definition 10 that

$$
\mathrm{C}_{1}=\mathrm{x} \sqsubseteq\left\langle\operatorname{eval}\left(\mathrm{S}_{1}, \mathrm{~s}\right), \mathrm{eval}\left(\mathrm{S}_{1}, \mathrm{t}\right)\right\rangle
$$

We have to prove that $\mathrm{C} \mathrm{S}_{1} \mathrm{C}_{1}$ and $\mathrm{C}_{1} \preceq \mathrm{C}_{2}$ which means that, by Definition 7 and Definition 11, we have to show that $c_{1}$ is simple and that

$$
\left\langle\operatorname{eval}\left(\mathrm{S}_{1}, \overline{\mathrm{s}}\right) \text {, eval }\left(\mathrm{S}_{1}, \mathrm{t}\right)\right\rangle \preceq\left\langle\operatorname{eval}\left(\mathrm{S}_{2}, \overline{\mathrm{s}}\right) \text {, eval }\left(\mathrm{S}_{2}, \mathrm{t}\right)\right\rangle \text {. }
$$

However, by Definition 5, if relation (10) holds, $c_{1}$ is simple. Thus, by the product of lattices (i.e., direct product), we just have to show that

$$
\begin{aligned}
& \operatorname{eval}\left(S_{1}, s\right) \preceq \operatorname{eval}\left(S_{2}, s\right) \text { and } \\
& \text { eval }\left(S_{1}, t\right) \preceq \operatorname{eval}\left(S_{2}, t\right) \text {. }
\end{aligned}
$$

Let $n$ (term) be the number of operators in term. We prove (i) by induction on $\mathrm{n}(\overline{\mathrm{s}})$. The proof of (ii) is similar and omitted.

- Base case $\mathrm{n}(\overline{\mathrm{s}})=0$. If $\overline{\mathrm{s}} \in \overline{\mathrm{Ls}}$, then, by Definition 10 , eval $\left(\mathrm{S}_{1}, \overline{\mathrm{s}}\right)=$ $\operatorname{eval}\left(S_{2}, \bar{s}\right)=\bar{s}$. If $\bar{s} \notin \overline{L^{s}}$, then $\bar{s}=\min (y)$ or $\bar{s}=\operatorname{val}(y)$ for some $y \in X$. Thus there exists $c_{y}=y \sqsubseteq\left\langle\overline{S_{y}}, t_{y}\right\rangle \in S_{1}$ and $c_{y}^{\prime}=y \sqsubseteq\left\langle\overline{S_{y}^{\prime}}, t_{y}^{\prime}\right\rangle \in S_{2}$ so that as, by hypothesis $\mathrm{S}_{1} \preceq \mathrm{S}_{2}$, we have

$$
\mathrm{S}_{1} \preceq \mathrm{S}_{2} \Rightarrow^{1} \mathrm{c}_{\mathrm{y}} \preceq \mathrm{C}_{\mathrm{y}}^{\prime} \Rightarrow^{2}\left\langle\overline{\mathrm{S}_{\mathrm{y}}}, \mathrm{t}_{\mathrm{y}}\right\rangle \preceq\left\langle\overline{\mathrm{S}_{\mathrm{y}}^{\prime}}, \mathrm{t}_{\mathrm{y}}^{\prime}\right\rangle \Rightarrow^{3} \overline{\mathrm{S}_{\mathrm{y}}} \preceq \overline{\mathrm{S}_{\mathrm{y}}^{\prime}} \text { and } \mathrm{t}_{\mathrm{y}} \preceq \mathrm{t}_{\mathrm{y}}^{\prime} \text {, }
$$

where $\Rightarrow^{1}$ follows from Definition $9, \Rightarrow^{2}$ from Definition 7 , and $\Rightarrow^{3}$ from the product of lattices (i.e., direct product) and Definition 5.

Suppose first that $\overline{\mathrm{s}}=\min (\mathrm{y})$. Then, by Definition 10 ,

$$
\operatorname{eval}\left(S_{1}, s\right)=S_{y} \text { and eval }\left(S_{2}, s\right)=\overline{S_{y}^{\prime}} \text {. }
$$

Therefore, by $(11)$, eval $\left(S_{1}, s\right) \preceq \operatorname{eval}\left(S_{2}, s\right)$.

Second, suppose that $\overline{\mathrm{s}}=\operatorname{val}(\mathrm{y})$. By $(9) \operatorname{eval}\left(\mathrm{S}_{2}, \overline{\mathrm{s}}\right) \in \overline{\mathrm{L}^{\mathrm{s}}}$ and by Definition 10, eval $\left(\mathrm{S}_{2}, \mathrm{~s}\right)=\overline{\mathrm{s}_{\mathrm{y}}^{\prime}}$ and $\mathrm{s}_{\mathrm{y}}^{\prime}=\mathrm{t}_{\mathrm{y}}^{\prime}$. Therefore, as $\mathrm{S}_{1}$ and hence $\mathrm{c}_{\mathrm{y}}$ are consistent, it follows from (11) and Lemma 1 that $s_{y}=t_{y}=s_{y}^{\prime}$. Thus, by Definition 10, eval $\left(S_{1}, s\right)=S_{y}$ so that eval $\left(S_{1}, s\right)=\operatorname{eval}\left(S_{2}, s\right)$.

- Nonbase case: $\mathrm{n}(\overline{\mathrm{s}})>0$. Suppose $\circ:: \mathrm{L}_{1}^{\mathrm{s}} \times \cdots \times \mathrm{L}_{\mathrm{n}}^{\mathrm{s}} \rightarrow \mathrm{L}^{\mathrm{s}}$ is a constraint operator (for $\mathrm{L}^{\mathrm{s}}$ ). Then, by Proposition $4(\mathrm{~b}), \bar{\sigma}$ is also a constraint operator (for $\left.\overline{L^{s}}\right)$. Then,

$$
\begin{aligned}
n(\bar{s})>0 \Rightarrow \bar{s}= & \overline{o\left(S_{1}, \ldots, S_{n}\right)}=^{1} \bar{o}\left(\bar{S}_{1}, \ldots, \bar{S}_{n}\right) \\
& \Rightarrow 4\left\{\begin{array}{l}
\operatorname{eval}\left(S_{1}, \bar{s}\right)=\bar{o}\left(\operatorname{eval}\left(S_{1}, \bar{s}_{1}\right), \ldots, \operatorname{eval}\left(S_{1}, \bar{S}_{n}\right)\right), \\
\operatorname{eval}\left(S_{2}, \bar{s}\right)=\bar{o}\left(\operatorname{eval}\left(S_{2}, s_{1}\right), \ldots, \operatorname{eval}\left(S_{2}, \bar{S}_{n}\right)\right),
\end{array}\right.
\end{aligned}
$$


where $={ }^{1}$ follows from Definition 3 and $\Rightarrow^{4}$ from Definition 10. By the inductive hypothesis,

$$
\operatorname{eval}\left(S_{1}, \bar{s}_{i}\right) \preceq \operatorname{eval}\left(S_{2}, \bar{S}_{i}\right), i \in\{1, \ldots, n\},
$$

and by Proposition 4(a), $\overline{0}$ is monotonic so that (i) holds.

\section{Proposition 2. (See Section 4.3.)}

Proof. By hypothesis $C \rightsquigarrow S_{1} C_{1}$ and $C \rightsquigarrow S_{2} C_{2}$ so that, by Definition $11, C_{1} \in$ $\mathcal{C}^{\mathrm{X}_{1}}$ and $\mathrm{C}_{2} \in \mathcal{C}^{\mathrm{X}_{2}}$ where $\mathrm{X}_{1} \subseteq \mathrm{X}$ and $\mathrm{X}_{2} \subseteq \mathrm{X}$ and

$$
\mathrm{C}_{1}=\left\{\mathrm{C}_{1} \mid \exists \mathrm{C} \in \mathrm{C} . \mathrm{C} \rightsquigarrow^{\mathrm{S}_{1}} \mathrm{C}_{1}\right\} \quad \text { and } \mathrm{C}_{2}=\left\{\mathrm{C}_{2} \mid \exists \mathrm{C} \in \mathrm{C} . \mathrm{C} \mathrm{S}_{2} \mathrm{C}_{2}\right\} \text {. }
$$

As $\mathrm{S}_{1} \preceq \mathrm{S}_{2}$, by Lemma 2 ,

$$
\forall C_{2} \in C_{2}: \exists C_{1} \in C_{1} \text { such that } C_{1} \preceq C_{2} \text {. }
$$

By Definition 7, if $c_{1} \preceq c_{2}$ then $c_{1}$ and $c_{2}$ are constrained on the same variable $x \in X$. Then, it follows from (12) that

$$
\mathrm{X}_{2} \subseteq \mathrm{X}_{1}
$$

Let $C_{1 x}$ and $C_{2 x}$ be the sets of constraints, in $C_{1}$ and $C_{2}$, respectively, with constrained variable $x \in X$ (note that $C_{1 x}$ and $C_{2 x}$ can be the empty set). It follows from (12) and (13) that, for each $c_{2} \in C_{2 x}$, there exists $c_{1} \in C_{1 x}$ such that $\mathrm{C}_{1} \preceq \mathrm{C}_{2}$.

Suppose that $c_{1 x}, c_{2 x}, c_{1 x}^{\prime}$, and $C_{2 x}^{\prime}$ are the constraints for $x \in X$ in the stores $S_{1}, S_{2}, S_{1}^{\prime}$, and $S_{2}^{\prime}$, respectively. By hypothesis $S_{1} \preceq S_{2}$ so that, by Definition 9 , $\mathrm{C}_{1 \mathrm{x}} \preceq \mathrm{C}_{2 \mathrm{x}}$. Since $\mathrm{S}_{1} \cup \mathrm{C}_{1} \mapsto \mathrm{S}_{1}^{\prime}$ and $\mathrm{S}_{2} \cup \mathrm{C}_{2} \mapsto \mathrm{S}_{2}^{\prime}$, by Definition 12,

$$
\begin{aligned}
& C_{1 x}^{\prime}=\cap_{L}\left(C_{1 x} \cup\left\{C_{1 x}\right\}\right), \\
& C_{2 x}^{\prime}=\cap_{L}\left(C_{2 x} \cup\left\{C_{2 x}\right\}\right) .
\end{aligned}
$$

As consequence of Definition 8 and contractance property of $\cap_{L}$,

$$
C_{1 x}^{\prime} \preceq c_{2 x}^{\prime}, \text { for each } x \in X .
$$

Therefore, by Definition $9, \mathrm{~S}_{1}^{\prime} \preceq \mathrm{S}_{2}^{\prime}$.

Lemma 3. Let $\mathrm{C} \in \mathcal{C}^{\mathrm{X}}$ and $\mathrm{S}, \mathrm{R} \in \mathcal{S S}^{\mathrm{X}}$. If $\mathrm{R}$ is a solution for $\mathrm{C} \cup \mathrm{S}$, then $\mathrm{R} \preceq \mathrm{S}$.

Proof. By Definition 13, $\mathrm{R}$ is consistent, and

$$
\mathrm{C} \cup \mathrm{S} \rightsquigarrow{ }^{\mathrm{R}} \mathrm{C}_{\mathrm{R}} \text { and } \mathrm{R} \cup \mathrm{C}_{\mathrm{R}} \mapsto \mathrm{R} \text {. }
$$

By Definition 11, if $C \cup S \rightsquigarrow{ }^{R} C_{R}$, then $C_{R}$ is equivalent to $C_{R}=C_{1} \cup C_{2}$ where

$$
\mathrm{C} \rightsquigarrow{ }^{\mathrm{R}} \mathrm{C}_{1} \text { and } \mathrm{S} \rightsquigarrow{ }^{\mathrm{R}} \mathrm{C}_{2} \text {, }
$$

and also

$$
\mathrm{C}_{2}=\left\{\mathrm{c}^{\prime} \mid \exists \mathrm{c} \in \mathrm{S} \cdot \mathrm{c} \rightarrow^{\mathrm{R}} \mathrm{c}^{\prime}\right\} .
$$

Observe that if $\mathrm{c}=\mathrm{x} \sqsubseteq\langle\overline{\mathrm{S}}, \mathrm{t}\rangle$ is a simple constraint and $\mathrm{x} \in \mathrm{V}_{\mathrm{L}}$ then, by Definition 7, $\langle\bar{s}, t\rangle \in R_{L}^{s}$ and, by Definition $5, \bar{s} \in \overline{L^{s}}$ and $t \in L^{s}$. Therefore, by 
Definition 10

$$
\operatorname{eval}(R, c)=x \sqsubseteq\langle\operatorname{eval}(R, \bar{s}), \operatorname{eval}(R, t)\rangle=x \sqsubseteq\langle\bar{s}, t\rangle=c .
$$

By Definition 11, this means that $\mathrm{C}{ }^{\mathrm{R}} \mathrm{C}$ if $\mathrm{c}$ is simple. Since $\mathrm{S}$ contains only simple constraints, then by Definition $11, \mathrm{C}_{2}=\mathrm{S}$.

Moreover, from (14), $R \cup C_{R} \mapsto R$ and, as shown previously, $C_{R}=C_{1} \cup C_{2}=$ $\mathrm{C}_{1} \cup \mathrm{S}$. Thus $\mathrm{R} \cup \mathrm{C}_{1} \cup \mathrm{S} \mapsto \mathrm{R}$ and, by Proposition $5, \mathrm{R} \preceq \mathrm{S}$.

Theorem 1 (Correctness). (See Section 5.1.)

Proof. Let $\mathrm{S}_{0}$ be the initial value of $\mathrm{S}$ and $\mathrm{C}=\mathrm{C} \cup \mathrm{S}_{0}$. Suppose that there are $\mathrm{k}$ iterations of the repeat loop and that, for each $\mathrm{i}$ where $1 \leq \mathrm{i} \leq \mathrm{k}, \mathrm{S}_{\mathrm{i}}$ is the value of the constraint store $S$ at Step (5), after completing ith iterations of the repeat loop.

Suppose first that a solution $\mathrm{R}$ (for $\mathrm{C} \cup \mathrm{S}_{0}$ ) exists. Then, by Definition $13, \mathrm{R}$ is consistent, $\mathrm{R} \in \mathcal{S S}^{\mathrm{X}}$ and

$$
\mathrm{C} \rightsquigarrow{ }^{\mathrm{R}} \mathrm{C}_{\mathrm{R}} \text { and } \mathrm{R} \cup \mathrm{C}_{\mathrm{R}} \mapsto \mathrm{R} \text {. }
$$

Note that, initially $\mathrm{S}_{0}=\mathrm{S} \in \mathcal{S S}^{\mathrm{X}}$. Then, by Lemma 3,

$$
\mathrm{R} \preceq \mathrm{S}_{0} \text {. }
$$

We show by induction on $\mathrm{i}$ that, after $\mathrm{i} \geq 0$ iterations of the repeat loop,

$$
\mathrm{R} \preceq \mathrm{S}_{\mathrm{i}} .
$$

The base case when $\mathrm{i}=0$ is given by (16). For the inductive step, suppose that there are at least $\mathrm{i}>0$ iterations of the repeat loop and that, after $\mathrm{i}-1$ steps, we have $\mathrm{R} \preceq \mathrm{S}_{\mathrm{i}-1}$. Then, in the ith iteration

$$
\begin{array}{ll}
\mathrm{C} \mathrm{S}_{\mathrm{i}-1} \mathrm{C}^{\prime} & \text { by Step (2); } \\
\mathrm{S}_{\mathrm{i}-1} \cup \mathrm{C}^{\prime} \mapsto \mathrm{S}_{\mathrm{i}}, & \text { by Step (4). }
\end{array}
$$

It follows from (15), (18), (19), and Proposition 2 that (17) holds.

Therefore, $R \preceq S_{k}$. As $R$ is consistent, by Proposition $6, S_{k}$ is consistent. However, the procedure terminates before the $k+1$ th iteration so that the test in Step (5) is true and we must have $S_{k}=S_{k-1}$. By (18) and (19)

$$
\mathrm{C} \rightsquigarrow \mathrm{S}_{\mathrm{k}-1} \mathrm{C}^{\prime} \text { and } \mathrm{S}_{\mathrm{k}-1} \cup \mathrm{C}^{\prime} \mapsto \mathrm{S}_{\mathrm{k}} \text {. }
$$

Thus, by Definition $13, S_{k}$ is a solution for $C$ (i.e., $C \cup S_{0}$ ). Moreover, if $R$ is another solution for $C \cup S_{0}$, then as $R \preceq S_{k}$ and as $R$ was any solution for $\mathrm{C} \cup \mathrm{S}_{0}, \mathrm{~S}_{\mathrm{k}}=\operatorname{mgs}\left(\mathrm{C} \cup \mathrm{S}_{0}\right)$.

Suppose next that there is no solution for $C \cup S_{0}$. Then $S_{k-1} \neq S_{k}$ or else, by (20), $S_{k}$ would be a solution. Thus, in this case, as the procedure terminates before the $k+1$ th iteration so that the test in Step (5) is true, we must have $S_{k}$ is inconsistent.

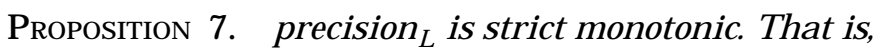

$$
\operatorname{precision}_{\mathrm{L}}(\mathrm{c}) \prec \operatorname{precision}_{\mathrm{L}}\left(\mathrm{C}^{\prime}\right) \text { if } \mathrm{c} \prec \mathrm{C}^{\prime} \text {. }
$$


Proof. Suppose that $c_{1}=x \sqsubseteq\left\langle\overline{a_{b}}, c_{d}\right\rangle$ and $c_{2}=x \sqsubseteq\left\langle\overline{a_{b}^{\prime}}, c_{d^{\prime}}^{\prime}\right\rangle$ where $c_{1} \prec c_{2}$. Then, we have to prove that

$$
\operatorname{precision}_{\mathrm{L}}\left(\mathrm{c}_{1}\right) \prec \operatorname{precision}_{\mathrm{L}}\left(\mathrm{c}_{2}\right) \text {, }
$$

which, by Definition 14, is equivalent to showing

$$
\left(\hat{a} \diamond_{L} c, b \diamond_{B} d\right) \prec\left(\hat{a}^{\prime} \diamond_{L} c^{\prime}, b^{\prime} \diamond_{B} d^{\prime}\right) .
$$

By hypothesis, $C_{1} \prec C_{2}$, so that by Definition 7

$$
\left\langle\overline{a_{b}}, c_{d}\right\rangle \prec\left\langle\overline{a_{b^{\prime}}^{\prime}}, c_{d^{\prime}}^{\prime}\right\rangle
$$

and, by the product of lattices (i.e., direct product), either

$$
\begin{aligned}
& \overline{a_{b}} \prec \overline{a_{b}^{\prime}} \text { and } c_{d} \preceq c_{d^{\prime}}^{\prime} \text { or } \\
& \overline{a_{b}} \preceq \overline{a_{b^{\prime}}^{\prime}} \quad \text { and } \quad c_{d} \prec c_{d^{\prime}}^{\prime} \text {. }
\end{aligned}
$$

If (22) holds, then, by the product of lattices (i.e., lexicographic product),

$$
\text { ( } \left.\hat{a} \prec \hat{a^{\prime}} \text { or } \hat{a}=\hat{a^{\prime}} \text { and } b \prec b^{\prime}\right) \text { and }\left(c \prec c^{\prime} \text { or } c=c^{\prime} \text { and } d \preceq d^{\prime}\right) \text {. }
$$

Similarly, if (23) holds, then,

$$
\text { ( } \hat{a} \prec \hat{a}^{\prime} \text { or } \hat{a}=\hat{a}^{\prime} \text { and } b \preceq b^{\prime} \text { ) and }\left(c \prec c^{\prime} \text { or } c=c^{\prime} \text { and } d \prec d^{\prime}\right. \text { ). }
$$

Therefore, $\hat{a} \prec \hat{a}^{\prime}$ and $c \preceq c^{\prime}, \hat{a}=\hat{a}^{\prime}$ and $c \prec c^{\prime}$, or $\hat{a}=\hat{a}^{\prime}$ and $c=c^{\prime}$. However, we have

$$
\begin{aligned}
& \left\{\begin{array}{l}
\hat{a} \prec \hat{a^{\prime}} \text { and } c \preceq c^{\prime} \\
\hat{a}=\hat{a^{\prime}} \text { and } c \prec c^{\prime}
\end{array}\right\} \Rightarrow^{1} \hat{a} \diamond_{L} c \prec \hat{a^{\prime}} \diamond_{L} c^{\prime} \Rightarrow^{2} \text { (21) holds, } \\
& \hat{a}=\hat{a^{\prime}} \text { and } c=c^{\prime}\left\{\begin{array}{l}
\Rightarrow^{3} b \prec b^{\prime} \text { and } d \preceq d^{\prime} \\
\Rightarrow^{4} b \preceq b^{\prime} \text { and } d \prec d^{\prime}
\end{array}\right\} \Rightarrow \Rightarrow^{1,5} \\
& \hat{a} \diamond_{L} C=\hat{a^{\prime}} \diamond_{L} c^{\prime} \text { and } b \diamond_{B} d \prec b^{\prime} \diamond_{B} d^{\prime} \Rightarrow^{2} \text { (21) holds, }
\end{aligned}
$$

where $\Rightarrow^{1}$ follows from strict monotonicity of $\diamond_{L}$ in Definition $14, \Rightarrow^{2}$ from the product of lattices (i.e., lexicographic product), $\Rightarrow^{3}$ from (24), $\Rightarrow^{4}$ from (25), and $\Rightarrow{ }^{5}$ from strict monotonicity of $\diamond_{B}$ in Definition 14 .

Theorem 2 (Termination). (SeeSection 5.2.)

Proof. Let $S_{0}$ be the initial value of $S$. Suppose there are at least $i \geq 0$ iterations of the repeat loop. If $i \geq 1$, let $S_{i} \in \mathcal{S S}^{X}$ be the constraint store at the end of the ith iteration. Suppose first that $S_{j}$ is inconsistent for some $j$, $0 \leq \mathrm{j} \leq \mathrm{i}$. Then either the test in Step (0) (if $\mathrm{j}=0$ ) or the test in Step (5) (if $\mathrm{j}>0$ ) fails and the procedure terminates after $\mathrm{i}=\mathrm{j}$ iterations. We now assume that $S_{i}$ is consistent for all $i \geq 0$ such that $S_{i}$ is defined.

For each $i \geq 0$ such that $S_{i}$ is defined and each $x \in X \cap V_{L}$ and $L \in \mathcal{L}$, let $C_{x}^{i}$ denote the simple (consistent) constraint in $S_{i}$. Also, for each $x \in X$ and some $\mathrm{L} \in \mathcal{L}$, let precision ${ }_{\mathrm{L}}\left(c_{x}^{\mathrm{i}}\right)=\left(\phi_{x}^{\mathrm{i}}, \psi_{x}^{\mathrm{i}}\right)$. For each $\mathrm{i} \geq 0$ such that $\mathrm{S}_{\mathrm{i}}$ is defined, let

$$
\begin{aligned}
& X_{i}=\left\{x \in X \mid \phi_{x}^{i}=T_{\Re^{+}}\right\}, \\
& Y_{i}=\left\{y \in X \mid \phi_{y}^{i} \prec T_{\Re^{+}}\right\} .
\end{aligned}
$$

ACM Transactions on Programming Languages and Systems, Vol. 26, No. 1, J anuary 2004. 
In the ith iteration we have, by Step (4), $S_{i-1} \cup C^{\prime} \mapsto S_{i}$ so that, by Proposition $5, S_{i} \preceq S_{i-1}$. Thus, by Definition 9, for each $x \in X, C_{x}^{i} \preceq C_{x}^{i-1}$, and, by Proposition $7,\left(\phi_{x}^{i}, \psi_{x}^{i}\right) \preceq\left(\phi_{x}^{i-1}, \psi_{x}^{i-1}\right)$. As the order is lexicographic, we have for all $X \in X, \phi_{x}^{i} \leq \psi_{x}^{i-1}$. Thus $X_{i} \subseteq X_{i-1}$ and, if $X_{i}=X_{i-1}$, then $Y_{i}=Y_{i-1}$. If no_difference ${ }_{\delta}\left(S_{i-1}, S_{i}\right)$ holds, then the test in Step (5*) is true and the procedure terminates. We now assume that the termination condition no_difference ${ }_{\delta}\left(S_{i-1}, S_{i}\right)$ does not hold. There are two cases:

(1) $X_{i} \subset X_{i-1}$;

(2) $\mathrm{X}_{\mathrm{i}}=\mathrm{X}_{\mathrm{i}-1}, \mathrm{Y}_{\mathrm{i}}=\mathrm{Y}_{\mathrm{i}-1}$ and $\exists \mathrm{y} \in \mathrm{Y}_{\mathrm{i}} \cdot\left(\phi_{\mathrm{y}}^{\mathrm{i}-1}, \psi_{\mathrm{y}}^{\mathrm{i}-1}\right)-\left(\phi_{\mathrm{y}}^{\mathrm{i}}, \psi_{\mathrm{y}}^{\mathrm{i}}\right)>(\varepsilon, 0)$.

As $\mathrm{X}_{i} \subset \mathrm{X}_{\mathrm{i}-1}$ can occur at most \# $\mathrm{X}_{0}$ different values for $\mathrm{i}$, we can assume that, for some iteration $j \geq 0, X_{i}=X_{j}$ for all $i \geq j$ and, for all $i>j$, Case (2) applies. Thus, for all $\mathrm{i}>\mathrm{j}, \mathrm{Y}_{\mathrm{i}}=\mathrm{Y}_{\mathrm{i}-1}$, so that, as $\phi_{x}^{\mathrm{i}} \leq \psi_{\mathrm{x}}^{\mathrm{i}-1}$ for all $\mathrm{x} \in \mathrm{Y}_{\mathrm{i}}$,

$$
\sum_{x \in Y_{i}} \phi_{x}^{i} \leq \sum_{x \in Y_{i-1}} \phi_{x}^{i-1} \text {. }
$$

At Step $\left(5^{\star}\right)$ of the ith iteration where $\mathrm{i}>\mathrm{j}$, either the repeat loop terminates or no_difference $\left(S_{i-1}, S_{i}\right)$ does not hold and thus, by Case (2),

$$
\sum_{\mathrm{x} \in \mathrm{Y}_{\mathrm{i}-1}} \phi_{\mathrm{x}}^{\mathrm{i}-1}-\sum_{\mathrm{x} \in \mathrm{Y}_{\mathrm{i}}} \phi_{\mathrm{x}}^{\mathrm{i}}>\varepsilon
$$

Let

$$
\mathrm{k}=\left\|\sum_{\mathrm{x} \in \mathrm{Y}_{\mathrm{j}}} \phi_{\mathrm{x}}^{\mathrm{j}} / \varepsilon\right\|+\mathrm{j},
$$

where $\|\mathrm{r}\|$ denotes the integer part of $\mathrm{r} \in \mathfrak{R}^{+}$. It follows that, if there is a kth iterations of the repeat loop, then $\sum_{\mathrm{x} \in \mathrm{Y}_{\mathrm{k}}} \phi_{\mathrm{x}}^{\mathrm{k}}<\varepsilon$ and hence no_difference $\left(\mathrm{S}_{\mathrm{k}-1}, \mathrm{~S}_{\mathrm{k}}\right)$ holds. Thus the procedure has at most $\mathrm{k}$ iterations.

Theorem 3 (Correctness in the Extended Schema). (SeSection 5.2.)

PRoof. Supposetheprocedureterminates after $k$ iterations with $\mathrm{S}_{\varepsilon}$ thefinal value of the constraint store S. It has already been shown in (17) of the proof of Theorem 1 that

$$
\mathrm{R} \preceq \mathrm{S}_{\varepsilon} .
$$

Thus, for all $c_{x} \in R$ and $C_{x}^{\prime} \in S_{\varepsilon}$, precision $\left(c_{x}\right) \preceq \operatorname{precision}\left(C_{x}^{\prime}\right)$. Let $\delta \in$ $\Re \mathcal{I}$ be the maximum of precision $\left(C_{x}^{\prime}\right)-\operatorname{precision}\left(c_{x}\right)$ for all $x \in X$. Then no_difference ${ }_{\delta}\left(S_{\varepsilon}, R\right)$ holds. Thus, by Definition $16, S_{\varepsilon}$ is an approximate solution for $\mathrm{C} \cup \mathrm{S}$.

\section{THEOREM 4. (SeeSection 5.2.)}

Proof. In the previous proof we have $\mathrm{R} \preceq \mathrm{S}_{\varepsilon}$ for any $\varepsilon \geq 0.0$. Therefore $\mathrm{R} \preceq \mathrm{S}_{\varepsilon_{1}}$ and $\mathrm{R} \preceq \mathrm{S}_{\varepsilon_{2}}$ ThuS, we just have to show that $\mathrm{S}_{\varepsilon_{1}} \preceq \mathrm{S}_{\varepsilon_{2}}$.

Supposethe procedures solve $e_{\varepsilon_{2}}(\mathrm{C}, \mathrm{S})$ and solve $\mathrm{\varepsilon}_{\varepsilon_{1}}(\mathrm{C}, \mathrm{S})$ terminate after $\mathrm{k}_{2}$ and $\mathrm{k}_{1}$ iterations, respectively. Therefore, the check, in Line $\left(5^{\star}\right)$, for the repeat loop for steps from 1 to $k_{2}$ must also succeed for $\varepsilon_{1}$. Thus $k_{1} \geq k_{2}$. 
Suppose that $S_{i}$ is the value of $S$ at the end of the ith iteration of the repeat loop $\left(1 \leq \mathrm{i} \leq \mathrm{k}_{1}\right.$ ) (so that $\mathrm{S}_{\mathrm{k}_{1}}=\mathrm{S}_{\varepsilon_{1}}$ and $\mathrm{S}_{\mathrm{k}_{2}}=\mathrm{S}_{\varepsilon_{2}}$ ). We show, by induction on $\mathrm{i}$, where $\mathrm{k}_{2} \leq \mathrm{i} \leq \mathrm{k}_{1}$, that

$$
\mathrm{S}_{\mathrm{i}} \preceq \mathrm{S}_{\varepsilon_{2}} \text {. }
$$

The base case when $\mathrm{i}=\mathrm{k}_{2}$ is obvious. For theinductivestep, supposethat $\mathrm{i}>\mathrm{k}_{2}$ and assume that $\mathrm{S}_{\mathrm{i}-1} \preceq \mathrm{S}_{\varepsilon_{2}}$. By Step (4) of the extended operational schema, we have

$$
\mathrm{S}_{\mathrm{i}-1} \cup \mathrm{C}^{\prime} \mapsto \mathrm{S}_{\mathrm{i}}
$$

at theend of theith iteration of the repeat loop. Thus, by Proposition $5, S_{i} \preceq S_{i-1}$ so that (26) holds. Therefore, letting $\mathrm{i}=\mathrm{k}_{1}$ we obtain $\mathrm{S}_{\varepsilon_{1}} \preceq \mathrm{S}_{\varepsilon_{2}}$.

\section{ACKNOWLEDGMENTS}

We would like to thank the referees of earlier versions of this paper. Their comments have hel ped us improve many aspects of this work.

\section{REFERENCES}

AïT-KACI, H. 1999. Warren's Abstract Machine: A Tutorial Reconstruction. The MIT Press, Cambridge, MA.

AlLen, J . 1983. Maintaining knowledge about temporal intervals. Commun. ACM 26, 11, 832843.

APT, K. 1999. The rough guide to constraint propagation. In 5th International Conference on Principles and Practice of Constraint Programming (CP'99, Alexandria, Virginia), J J J affar, Ed. Lecture Notes in Computer Science, vol. 1713. Springer-Verlag, Berlin, Germany, 1-23.

APT, K. 2000. The role of commutativity in constraint propagation algorithms. ACM Trans. Programm. Lang. Syst. 22, 6, 1002-1036.

BaAder, F. AND Schulz, K. 1995. On the combination of symbolic constraints, solution domains and constraints solvers. In 1st International Conference on Principles and Practice of Constraint Programming (CP'95, Cassis, France), U. Montanari and F. Rossi, E ds. Lecture Notes in Computer Science, vol. 976. Springer-Verlag, Berlin, Germany, 380-397.

Barth, P. And BockmaYr, A. 1996. Modelling 0-1 problems in $\operatorname{CLP}(\mathcal{P B})$. In 2nd International Conference on the Practical Application of Constraint Technology (PACT'96), M. Wallace, Ed. Prolog Management Group, London, U.K., 1-9.

Benhamou, F. 1995. Interval constraint logic programming. In Constraint Programming: Basics and Trends, A. Podelski, Ed. Lecture Notes in Computer Science, vol. 910. Springer-Verlag, Berlin, Germany, 1-21.

Benhamou, F., Goualard, F., Granvilliers, L., and Puget, J .-F. 1999. Revising hull and box consistency. In 16th International Conference on Logic Programming (ICLP'99, Las Cruces, New Mexico,) D. De Schreye, Ed. The MIT Press, Cambridge, MA, 230-244.

Benhamou, F. and Older, W. 1997. Applying interval arithmetic to real, integer and Boolean constraints. J . Logic Programm. 32, 1 (J uly), 1-24.

Bırkhoff, G. 1967. Lattice Theory, 3rd ed. Coloquium Publications, vol. XXV. American Mathematical Society, Providence RI.

BISTARELLI, S., MONTANARI, U., AND RossI, F. 1995. Constraint solving over semirings. In 14th I nternational J oint Conferenceon Artificial I ntelligent (IJ CAI '95, Québec, Canada). Morgan Kaufman, San Francisco, CA, 624-630.

Carlsson, M., Ottosson, G., ANd Carlson, B. 1997. An open-ended finite domain constraint solver. In 9th I nternational Symposium on Programming Languages: I mplementations, Logics and Programs (PLILP'97, Southampton, U.K.), U. Montanari and F. Rossi, Eds. Lecture Notes in Computer Science, vol. 1292. Springer-Verlag, Berlin, Germany, 191-206.

ACM Transactions on Programming Languages and Systems, Vol. 26, No. 1, J anuary 2004. 
Cleary, J. 1987. Logical arithmetic. Fut. Comput. Syst. 2, 2, 125-149.

Codognet, P. AND DIAZ, D. 1993. Boolean constraint solving using dp(FD). In 1993 International Symposium on Logic Programming (ILPS'93 Vancouver, British Columbia, Canada), D. Miller, Ed. The MIT Press, Cambridge, MA, 525-539.

Codognet, P. And Diaz, D. 1994. Clp(B): combining simplicity and efficiency in Boolean constraint solving. In 6th International Symposium on Programming Languages I mplementation and Logic Programming (PLILP'94, Madrid, Spain). Lecture Notes in Computer Science, vol. 844. SpringerVerlag, Berlin, Germany, 244-260.

Codognet, P. And DiAZ, D. 1996a. Compiling constraints in clp(FD). J . Logic Programm. 27, 3, 185-226.

Codognet, P. And Diaz, D. 1996b. A simple and efficient Boolean solver for constraint logic programming. J . Automat. Reason. 17, 1, 97-129.

Davey, B. And Priestley, H. 1990. Introduction to Lattices and Order. Cambridge University Press, Cambridge, England.

Diaz, D. And Codognet, P. 1993. A minimal extension of the WAM for clp(FD). In 10th International Conferenceon Logic Programming (ICLP'93, Budapest, Hungary), D. Warren, Ed. The MIT Press, Cambridge, MA, 774-790.

Diaz, D. And Codognet, P. 2001. Design and implementation of the GNU prolog system. J . Funct. Logic Programm. 2001, 6 (Oct.).

Fernández, A. 2000. clp $(L)$ version 0.21, user manual. Available online at http://www.lcc.uma. es/ afdez/generic.

FeRnÁndez, A. 2002. A generic, collaborative framework for interval constraint solving. Ph.D. dissertation, E.T.S.I.I., Dpto. Lenguajes y Ciencias de la Computación, University of Málaga, Spain. English version available online at http://www.lcc.uma.es/ afdez/papers.html.

FERnÁndez, A. AND HILL, P. 1999a. Interval constraint solving over lattices using chaotic iterations. In ERCIM/ COMPULOG Workshop on Constraints, K.Apt, A. Kakas, E. Monfroy, and F. Rossi, Eds. Dept., of Computer Science, University of Cyprus, Paphos, Cyprus. Available online at http://www.cwi.nl/ERCIM/WG/Constraints/Workshops/Workshop4/Program.

FERnÁndez, A. AND HILL, P. 1999b. An interval lattice-based constraint solving framework for lattices. In 4th International Symposium on Functional and Logic Programming (FLOPS'99, Tsukuba, J apan), A. Middeldorp and T. Sato, Eds. Lecture N otes in Computer Science, vol. 1722. Springer-Verlag, Berlin, Germany, 194-208.

FERNÁNDEZ, A. AND HILL, P. 2000. A comparativestudy of eight constraint programming languages over the Boolean and finite domains. Constraints 5, 3, 275-301.

FRÜHWIRTH, T. 1998. Theory and practice of constraint handling rules. J . Logic Programm. 37, 95-138.

Georget, Y. And Codognet, P. 1998. Compiling semiring-based constraints with clp(FD,S). In 4th International Conference on Principles and Practice of Constraint Programming (CP'98, Pisa, I taly), M. Maher and J .-F. Puget, Eds. Lecture Notes in Computer Science, vol. 1520. SpringerVerlag, Berlin, Germany, 205-219.

GeRvet, C. 1997. Interval propagation to reason about sets: Definition and implementation of a practical language. Constraints 1, 3, 191-244.

Goualard, F., Benhamou, F., and Granvilliers, L. 1999. An extension of the WAM for hybrid interval solvers. J. Funct. Logic Programm. 1999, 1 (April), 1-36. Special issue of Workshop on Parallelism and Implementation Technology for (Constraint) Logic Programming Languages.

HICKEY, T. 2000. CLIP: a CLP(Intervals) dialect for metalevel constraint solving. In 2nd International Workshop on Practical Aspects of Declarative Languages (PADL'2000, Boston, Massachusetts), E. Pontelli and V. Costa, E ds. Lecture Notes in Computer Science, vol. 1753. SpringerVerlag, Berlin, Germany, 200-214.

HofSTEDT, P. 2000. Better communication for tighter cooperation. In 1st I nternational Conference on Computational Logic (CL'2000, London, U.K.), J. W. Lloyd, V. Dahl, U. Furbach, M. Kerber, K. Lau, C. Palamidessi, L. Pereira, Y. Sagiv, and P. J . Stuckey, Eds. Lecture N otes in Computer Science, vol. 1861. Springer-Verlag, Berlin, Germany, 342-357.

If/Prolog. 1994. IF/ Prolog V5.0A, Constraints Package. Siemens Nixdorf I nformationssysteme AG, Munich, Germany. 
ISO/IEC. 1995. ISO/IEC 13211-1: 1995 Information Technology-Programming LanguagesProlog_Part 1: General Core International Standard Organization, Geneva, Switzerland.

J affar, J., Michaylov, S., Stuckey, P., And YaP, R. 1992. The CLP( $\Re)$ language and system. ACM Trans. Programm. Lang. Syst. 14, 3, 339-395.

Le Provost, T. and Wallace, M. 1993. Generalized constraint propagation over the CLP scheme. J. Logic Programm. 16, 3 (Aug.), 319-359.

LEe, J. AND VAN Emden, M. 1993. Interval computation as deduction in CHIP. J ournal Logic Programm. (Special Issue on Constraint Logic Programming) 16, 3-4, 255-276.

Moore, R. 1966. Interval Analysis. Prentice Hall, Englewood Cliffs, NJ .

N'Dong, S. 1997. Prolog IV ou la programmation par contraintes selon Prologl A. In Sixièmes J ournées Francophones de Programmation Logique et Programmation par Contraintes (J FPLC'97). Edition HERMES, Orléans, France, 235-238.

OldeR, W. 1989. Interval arithmetic specification. Tech. Rep. Bell-Northern, Research Computing Research Laboratory, Ottawa, Ontario, Canada.

Older, W. And Vellino, A. 1993. Constraint arithmetic on real intervals. In Constraint Logic Programming: Sel ected Research, F. Benhamou and A. Colmesauer, Eds. The MIT Press, Cambridge, MA, 175-195.

Refalo, P. and Van Hentenryck, P. 1996. CLP( $\left.\Re_{\text {lin }}\right)$ revised. In J oint International Conference and Symposium on Logic Programming (J ICSLP'96, Bonn, Germany), M. Maher, Ed. The MIT Press, Cambridge, MA, 22-36.

Schiex, T., Fargier, H., and Verfaillie, G. 1995. Valued constraint satisfaction problems: Hard and easy problems. In 14th International J oint Conference on Artificial Intelligent (IJ CAI'95, Québec, Canada). Morgan Kaufman, San Francisco, CA, 631-637.

Sicstus Manual. 1994. SICStus Prolog User's Manual, Release 3\#5. The Intelligent Systems Laboratory, Swedish Institute of Computer Science. Web site: http://www.sics.se.

Sidebottom, G. and Havens, W. 1992. Hierarchical arc consistency for disjoint real intervals in constraint logic programming. Computat. Intell. 8, 4, 601-623.

SLAVÍk, V. 1986. A note on the lattice of intervals of a lattice. Algebra Universalis 23, 1, 22-23.

Van Hentenryck, P., Saraswat, V., and Deville, Y. 1998. Design, implementation and evaluation of the constraint language cc(FD). J . Logic Programm. 37, 1-3 (Oct.), 139-164.

WALINSKY, C. 1989. CLP $\left(\Sigma^{*}\right)$ : Constraint logic programming with regular sets. In 6th International Conference on Logic Programming (ICLP'89, Lisbon, Portugal), G. Levi and M. Martelli, Eds. The MIT Press, Cambridge, MA, 181-196.

Received December 2000; revised March 2002; accepted March 2003 